\title{
Replica theory of the rigidity of structural glasses
}

\author{
Hajime Yoshino \\ Department of Earth and Space Science, Faculty of Science, Osaka University, Toyonaka 560-0043, Japan
}

We present a first principle scheme to compute the rigidity, i. e. the shear-modulus of structural glasses at finite temperatures using the cloned liquid theory, which combines the replica theory and the liquid theory. With the aid of the replica method which enables disentanglement of thermal fluctuations in liquids into intra-state and inter-state fluctuations, we extract the rigidity of metastable amorphous solid states in the supercooled liquid and glass phases. The result can be understood intuitively without replicas. As a test case, we apply the scheme to the supercooled and glassy state of a binary mixture of soft-spheres. The result compares well with the shear-modulus obtained by a previous molecular dynamic simulation. The rigidity of metastable states is significantly reduced with respect to the instantaneous rigidity, namely the Born term, due to non-affine responses caused by displacements of particles inside cages at all temperatures down to $T=0$. It becomes nearly independent of temperature below the Kauzmann temperature $T_{\mathrm{K}}$. At higher temperatures in the supercooled liquid state, the non-affine correction to the rigidity becomes stronger suggesting melting of the metastable solid state. Inter-state part of the static response implies jerky, intermittent stress-strain curves with static analogue of yielding at mesoscopic scales.

PACS numbers: 61.43.Fs 


\section{INTRODUCTION}

Rigidity is a distinct character of solids including crystals and glasses [1]. It is quantified unambiguously by the shear-modulus [2] which represents strength of materials against shear deformation, which changes the shape of the containers but not their volume. The shear-modulus is finite in solids but zero in gasses and liquids so that, much like the order parameters, it distinguishes solids from other states of matters, in sharp contrast to the bulk modulus which is finite even in gasses. We denote the shear-modulus simply as rigidity in the present paper. It is the rigidity of a Goldstone mode which reflects the breaking of the translational symmetry much as the rigidity of the spin-wave in ferromagnets which reflects the breaking of the rotational symmetry. The significance of the shear-modulus can be hardly appreciated at the level of the macroscopic elastic theory which treats all elastic constants on the same footing. We must go back to the microscopic scales to study the mechanism of the rigidity of solids. Unfortunately the rigidity of solids is usually taken for granted and regarded merely as a fitting or adjustable parameter. In the present paper we dig into this basic problem and attempt to develop a theoretical approach to compute the shear-modulus of structural glasses at finite temperatures starting from microscopic Hamiltonians. Microscopic computation of the rigidity of structural glasses would be useful also for practical applications of amorphous materials.

A class of amorphous solid or glasses can be obtained through supercooling liquids. First one cools a liquid down to the supercooled liquid state, the metastable liquid phase below the melting transition temperature $T_{\mathrm{m}}$. A supercooled liquid behaves as a visco-elastic material. On one hand the system behaves as a solid with a finite effective rigidity for a long time before it flows. Within this time scale, which is called as the $\beta$-regime, the configuration of the particles does not evolve much but the particles fluctuate mostly within narrow spaces around themselves called as cage created by the surrounding other particles. On the other hand the system behaves as a fluid with high viscosity at longer time scales called as the $\alpha$-regime. In this regime the configuration of the cages themselves are reorganized. The two qualitatively different relaxation mechanisms manifest themselves as two step relaxations, i. e. $\alpha$ and $\beta$ relaxations, in various observables such as the intermediate scattering function, dielectric susceptibility and e.t.c. [3]. Upon lowering the temperature, the viscosity or the structural relaxation time $\tau_{\alpha}$ increases enormously. Eventually $\tau_{\alpha}$ exceeds the laboratory time scale at some glass transition temperature $T_{\mathrm{g}}$ and the system falls out-of equilibrium. As the result we are left with a piece of glass with a finite rigidity, which behaves as a solid in all practical means. This is the glass transition observed in practice. Thus the rigidity of glasses is completed by enormous increase of the viscosity in sharp contrast to crystals where the rigidity is established abruptly by 1st order phase transitions with no appreciable precursors. The rich visco-elastic or rheological properties, which are absent in crystals, give distinct flavor to supercooled liquids and glasses and make them useful in practical applications. Apart from the interests to the outstanding question that whether an ideal, thermodynamic glass transition exists or not, development of first principle, microscopic theories to describe such an out-of equilibrium amorphous state of matters is strongly desired.

Among various theoretical proposals [4], the Random First Order Transition (RFOT) theory [5] 9] provides a useful working ground to study the supercooled liquids and amorphous solids in a unified manner. The basic scenario of the RFOT theory goes as follows at the mean field level. By lowing the temperature down to the so called dynamical transition temperature $T_{\mathrm{d}}$, exponentially larger number of metastable amorphous solid states emerge so that the structural entropy or the complexity becomes finite. The ideal, equilibrium glass transition takes place at the putative Kauzmann temperature $T_{\mathrm{K}}$ due to the entropy crisis mechanism [10]. Within the RFOT theory, $T_{\mathrm{d}}$ is equivalent to the critical temperature $T_{\mathrm{c}}$ predicted by the mode coupling theory (MCT) where $\tau_{\alpha}$ diverges [11, 12]. As is well known, the dynamical transition itself happens only at the mean-field level and $T_{\mathrm{d}}$ would remain in real systems at most as a crossover temperature. The metastable solid states can have only finite life times in finite dimensional systems. True divergence of the viscosity, if any, may occur approaching $T_{\mathrm{K}}$ as envisioned by Adam, Gibbs and DiMarzio 13, 14]. An important concern of the RFOT theory is to seek for a theoretical foundation of the scenario [7, 9, 15 17].

At the mean-field level, the RFOT theory is backed up by the cloned liquid method [18 21] and the mode-couping theory (MCT) [11, 12]. Consistency between the two approaches is currently investigated intensively [22]. The cloned liquid method provides a first principle scheme to compute the equilibrium and quasi-equilibrium properties of the supercooled liquids and amorphous solids starting from microscopic Hamiltonians with the help of the liquid theory [23] combined with the replica method [24, 25]. In the nutshell the cloned liquid theory, in its simplest formulations, views the metastable amorphous solids through an effective Einstein model in which each particle is subjected to a virtual Hookian spring with one end fixed at the mean position of each particle. The configuration of the latter is just that of a liquid. The virtual Hookian spring is meant to mimic the cage effect: the spring constant is inversely proportional to the size of the cage $A$, which is the order parameter of the theory. Physically, existence of amorphous states with finite cage size $A$ means breaking of the translational symmetry.

In the present paper we study rigidity of structural glasses against shear at finite temperatures using the fluctuation formula of the rigidity [26]. We evaluate it using the cloned liquid theory. This would amount to a formulation of an effective Debye model starting from the effective Einstein model, which is a necessary step to go beyond the mean-field 
theory. Long wave length elastic deformations are the essential low energy excitations in solids and play important roles much as spin-waves in ferromagnets. For example localized plastic events which are elementary steps of the flow in glasses 27 29] are known to accompany smooth elastic deformations around them, which naturally implies elastic free-energy barriers and interactions with each other through the long-ranged elastic couplings [30 33].

We show that the cloned liquid method enables decomposition of a generic response function into two parts: the intra-state responses corresponding to the $\beta$-relaxation and inter-state responses, which correspond to the $\alpha$ relaxation. Based on this scheme we compute the intra-state rigidity $\hat{\mu}$, which presumably represents the effective rigidity of supercooled liquids and the rigidity of glasses. On the other hand, the inter-state fluctuation reduces the rigidity down to 0 . In general, it is reasonable to expect that the $\beta$-relaxation only weakly changes between ideal equilibrium and out-of equilibrium situations while the $\alpha$-relaxation strongly changes [34]. Thus the quasi-static approximation for the intra-state responses would be valid for both the equilibrated supercooled liquids and out-of equilibrium glasses.

We find that the intra-state rigidity $\hat{\mu}$, i. e. the rigidity of the metastable glassy states is significantly reduced with respect to the Born term [35] which describes the instantaneous, affine response by a non-affine correction term which represents stress relaxations caused by relaxation of particles inside cages, i. e. the $\beta$-relaxation, at all temperatures down to the zero temperature limit. Our result implies a characteristic temperature dependence of the intra-state rigidity. On one hand, it is nearly constant below the Kauzmann temperature $T_{\mathrm{K}}$. On the other hand, it strongly depends on the temperature above $T_{\mathrm{K}}$. To test our scheme, we applied the method to a binary mixture of soft spheres. We found the theoretical prediction compares well with the previous result by Barrat et. al. (1988) [36] obtained by a molecular dynamic simulation.

An interesting general problem is how glasses melt. It has been proposed a long time ago by Born [37] that melting of solids may be signaled by vanishing of the rigidity. Although this rigidity crisis scenario obviously does not apply to the equilibrium liquid-solid transitions which are 1st order phase transitions, whether it is relevant for the melting of superheated metastable crystals approaching the spinodal temperature $T_{\mathrm{s}}$ from below is an intriguing question [38 41]. Interestingly enough, there is an intimate analogy [42] between the melting of metastable amorphous solids at the dynamical transition temperature $T_{\mathrm{d}}$ (or the MCT critical temperature $T_{\mathrm{c}}$ ) and the melting of superheated metastable crystals at $T_{\mathrm{s}}$. At the mean-field level $T_{\mathrm{d}}$ is regarded precisely as the temperature above which the metastable amorphous solids states become absent in equilibrium. Moreover it has been pointed out that the underlying mechanism of the dynamical critical phenomena found by MCT is the qualitative change of the free-energy landscape at around $T_{\mathrm{d}}$ [8, 43]. Then an interesting question is whether the rigidity crisis scenario also applies to the melting of the amorphous solids.

For clarity let us note that there are important classes of amorphous solids other than the glasses obtained by supercooling simple liquids. In a class of systems including gels, polymer glasses and rubbers, formation of disordered networks of molecules and colloids are important. Another important class is the granular matters which are athermal, i. e. the temperature play no essential roles. How the rigidity emerge in these amorphous systems have also attracted a lot of interests and some microscopic theoretical approaches have been developed [44 48].

The organization of the paper is as follows. In the next section, we discuss the background of the present work with some short reviews on related works, basic concepts and prepare basic tools needed in the analysis of the rigidity. In sec. III] we develop our scheme to compute the rigidity of structural glasses and apply it to the case of a binary mixture of soft-spheres. Finally in sec. IV and $\mathrm{V}$ we summarize the preset work and discuss some related problems. In the appendices we present some technical details. The brief account of the present work has been reported in [49].

\section{THE BACKGROUND}

In this section first we discuss some basic aspects of response to externally induced shear deformations in sec. ЏA. Then in sec. IIB we discuss a generic mean-field picture which suggests an intimate connection between the order parameter and rigidity in solids. Finally we review the cloned liquid method in sec. IIC

\section{A. Linear response to shear}

\section{Response to shear : a paradox and a lesson}

A material is said to be in a solid state if its rigidity (shear-modulus) $\mu$ is positive. Let us consider $N$ particles put in rectangular container which can be deformed by simple shear. Under a simple shear of shear-strain $\gamma$, the volume $V$ (and thus the number density $\rho=N / V$ of the particles) remains unchanged and only the shape of the container changes. (See. Fig. 11) 
Let us consider how much change occurs in the free-energy $F$ of the system by the simple shear. Assuming that the free-energy $F=N f$ is an analytic function of the shear-strain $\gamma$, we can expand the free-energy par particle $f(\gamma)=F(\gamma) / N$ as

$$
f(\gamma)=f(0)+\sigma \gamma+\frac{\gamma^{2}}{2} \mu+\ldots
$$

where we find the stress $\sigma$ and the rigidity $\mu$ as,

$$
\sigma=\frac{d f}{d \gamma} \quad \mu=\frac{d^{2} f}{d \gamma^{2}} .
$$

However, there is a plain fact that thermodynamic free-energy (par particle) $f$ should not depend on the shape of the container (remember that the density remains unchanged under the simple shear deformation),

$$
\lim _{N \rightarrow \infty} F(\gamma) / N=\text { const. }
$$

Thus we conclude that rigidity defined in the thermodynamic sense must always be zero, whether the system is a liquid or a solid [50] ! Apparently this goes against to our basic intuition that in solids $\mu>0$.

The paradox described above suggests that the thermodynamic limit $N \rightarrow \infty$ and small shear-strain limit $\gamma \rightarrow 0$ do not commute in solids: linear response theory which is built in the $\operatorname{limit}_{\lim } \rightarrow \infty \lim _{\gamma \rightarrow 0}$ fails to predict what actually happens in the thermodynamics $\lim _{\gamma \rightarrow 0} \lim _{N \rightarrow \infty}$. The breakdown of the commutation of the two limits is a consequence of the breaking of the transnational symmetry in solids. Physically the break down of the linear response theory means that as a system becomes a solid, not only the elasticity but plasticity must emerge simultaneously. The plasticity, which means non-linear responses like yielding or stress drops, recovers the translational symmetry at the macroscopic level required by the thermodynamics. In this respect, the idealized elastic bodies which appear in macroscopic continuum descriptions are purely hypothetical and thermodynamically unsound objects. In the present paper we actually limit ourselves to the linear response theory but we shall always keep in our mind the fact that the linear response theory must fail.

Finally let us note that we strictly consider shear-strain control protocols instead of shear stress control protocols in the present paper. In the latter case, static formulations are impossible and one essentially studies the rheology where one finds that "everything flows under shear". Physically the fact that even perfect crystals flow [51] is intimately related to the static paradox discussed above and the plasticity is the mechanism of the flow of solids under shear.

\section{Static fluctuation formula}

Let us consider a generic system of $N$ particles $(i=1,2, \ldots, N)$ of mass $m$ at position $\mathbf{r}_{i}=\left(x_{i}, y_{i}, z_{i}\right)$ in the laboratory frame. More generally we denote components of a position vector $\mathbf{r}_{i}$ in a $d$-dimensional space as $x_{i}^{\mu}$ with $\mu=1,2, \ldots, d$. We assume the Hamiltonian is given by,

$$
\mathcal{H}=K+U \quad K=\sum_{i=1}^{N} \frac{\mathbf{p}_{i}^{2}}{2 m} \quad U=\sum_{i<j} v\left(r_{i j}\right)
$$

where $\mathbf{r}_{i j} \equiv \mathbf{r}_{i}-\mathbf{r}_{j}$ and $r_{i j} \equiv\left|\mathbf{r}_{i j}\right|$ is the distance between the $i$-th and $j$-th particles. The term $K$ is the kinetic energy with $\mathbf{p}_{i}$ being the momentum of the $i$-th particle. For the potential energy $U$ we assume the simple two body interactions just for the sake of simplicity.
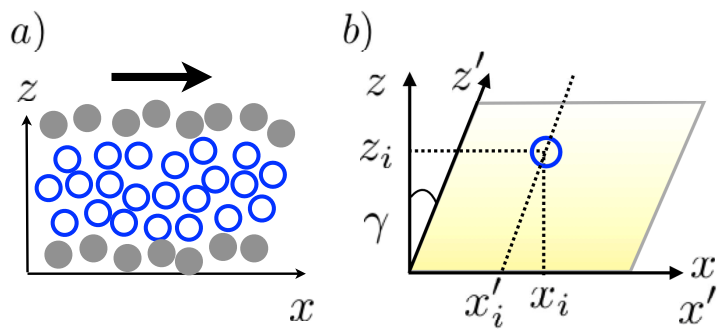

FIG. 1. Schematic picture of the system under static shear. a) The mobile particles (open circles) are bounded by "wall particles" (filled circle). b) Laboratory frame $(x, y, z)$ and sheared frame $\left(x^{\prime}, y^{\prime}, z^{\prime}\right)$. 
The free-energy of the system at temperature $T$ can be written in terms of the partition function $Z$ of the canonical ensemble as,

$$
-\beta F(T, \mathcal{V})=\log Z \quad Z=\frac{1}{N !} \int_{\mathcal{V}} \prod_{i=1}^{N} \frac{d^{d} r_{i}}{\Lambda^{d}} \exp \left(-\beta \sum_{i<j} v\left(r_{i j}\right)\right)
$$

where $\beta=1 / k_{\mathrm{B}} T$ is the inverse temperature, $\Lambda$ is the thermal de Brogile length $\Lambda=h / \sqrt{2 \pi m k_{\mathrm{B}} T}$ with $k_{\mathrm{B}}$ being the Boltzmann's constant. In Eq. (5), the symbol $\mathcal{V}$ is meant to represent symbolically the integration volume, i. e. the container of the system including not only its volume $V$ but also its shape, which will be important in the following.

In order to study the rigidity against simple shear deformation, we consider a container with two boundary walls which are normal to the $z$-axis and separated from each other by distance $L_{z}$ as shown in Fig 1 To impose a shearstrain $\gamma$ on the system, we simply displace the top wall by an amount $\gamma L_{z}$ into $x$-direction. The shear-deformation changes the boundary of the integration volume in Eq. (5). Let us denote the deformed boundary symbolically as $\mathcal{V}(\gamma)$. However, note that the volume of the system $V$ and thus the number density $\rho=N / V$ remain constant under this simple shear deformation.

Now we wish to find an expansion of the free-energy in power series of the shear-strain $\gamma$ as Eq. (1). To this end, it is convenient to change the integration variables to those of a sheared frame with $x^{\prime}, y^{\prime}$ and $z^{\prime}$ which are related to the original laboratory frame as, $(x, y, z)=\left(x^{\prime}+z^{\prime} \gamma, y^{\prime}, z^{\prime}\right)$ (See Fig. 1). Note that the boundary of the original integration variables $\mathcal{V}(\gamma)$ evolves with the shear-strain $\gamma$ but that for the new variables remains the same as the original one $\mathcal{V}(0)=\mathcal{V}$. But now we have to express the Hamiltonian, which is written in terms of the original coordinates, by the new ones. This can be done by simply expanding the Hamiltonian in power series of the shear-strain $\gamma$ assuming that it is small. In practice it is useful to notice,

$$
\frac{d}{d \gamma}=\sum_{i<j} z_{i j} \frac{\partial}{\partial x_{i j}}
$$

where $x_{i j} \equiv x_{i}-x_{j}$ and $z_{i j} \equiv z_{i}-z_{j}$. As the result, one easily finds a microscopic expression of the shear-stress defined in Eq. (2),

$$
\sigma=\left.\frac{1}{N} \frac{d U}{d \gamma}\right|_{\gamma=0}=\frac{1}{N} \sum_{i<j} \sigma\left(\mathbf{r}_{i j}\right) \quad \sigma\left(\mathbf{r}_{i j}\right)=\left.\frac{d v\left(r_{i j}\right)}{d \gamma}\right|_{\gamma=0}=\left.r v^{(1)}(r)\right|_{r=r_{i j}} \hat{x}_{i j} \hat{z}_{i j}
$$

where $\hat{x}_{i j} \equiv\left(x_{i}-x_{j}\right) / r_{i j}$ and $\hat{z}_{i j} \equiv\left(z_{i}-z_{j}\right) / r_{i j}$, and $v^{(n)} \equiv d^{n} v(r) / d r^{n}$. Similarly the explicit expression of the rigidity defined in Eq. (2) is found as [26],

$$
\mu=\langle b\rangle-N \beta\left[\left\langle\sigma^{2}\right\rangle-\langle\sigma\rangle^{2}\right]
$$

with

$$
b=\left.\frac{1}{N} \frac{d^{2} U}{d \gamma^{2}}\right|_{\gamma=0}=\frac{1}{N} \sum_{i<j} b\left(\mathbf{r}_{i j}\right) \quad b\left(\mathbf{r}_{i j}\right)=\left.\frac{d^{2} v\left(r_{i j}\right)}{d \gamma^{2}}\right|_{\gamma=0}=\hat{z}_{i j}^{2}\left[r^{2} v^{(2)}(r) \hat{x}_{i j}^{2}+r v^{(1)}(r)\left(1-\hat{x}_{i j}^{2}\right)\right]_{r=r_{i j}}
$$

In Eq. (8) $\langle\ldots\rangle$ denotes the thermal average evaluated with zero shear-strain $\gamma=0$,

$$
\langle\ldots\rangle=Z^{-1} \int \prod_{i=1}^{N} \frac{d^{d} r_{i}}{\Lambda^{d}} \exp \left(-\beta \sum_{i<j} v\left(r_{i j}\right)\right) \ldots
$$

The formula Eq. (8) is the static fluctuation formula of the rigidity [26]. The 1st term $b$ on the r. h. s of Eq. (8) is the so called Born term [35]. It represents the affine response of the system against shear, which is finite even in simple liquids. The 2 nd term is the non-affine correction term due to stress relaxations which play crucial roles as we discuss in the present paper. Let us call it as the fluctuation term in the following. The difference of the nature of the affine and non-affine terms will become more clear shortly by discussing the dynamic response against shear. It may be instructive to note that quite analogous fluctuation formula is known for the rigidity of spin-waves in ferromagnets or the helicity modulus of superconductors [52] where the non-affine part is represented by correlation functions of spin or super currents.

In liquids, the rigidity $\mu$ vanishes. It means an exact cancellation of the Born term and the fluctuation terms. The exact cancellation may look somewhat surprising but it is a direct consequence of the translational symmetry in liquids. 
The vanishing of the rigidity in liquids can also be seen formally by considering Mayer expansions, i. e. expansions in power series of the number density $\rho=N / V$, of the free-energy of liquids. Such an expansion should converge in liquids just like high temperature expansions are convergent in paramagnets. At each order in the expansion, one finds finite sized clusters. An infinitesimal shear-deformation, which is just an infinitesimal change on the boundary condition, should not change the contribution from the finite sized cluster to the free-energy per unit volume in the thermodynamic limit $V \rightarrow \infty$. Thus the rigidity is zero at each order of the density in the thermodynamic limit. The vanishing of the rigidity at the level of linear response means the two limits $V \rightarrow \infty$ and $\gamma \rightarrow 0$ do commute in liquids (see sec. [A 1). Similarly one can consider high temperature expansions in spin systems by which one arrives at the same conclusion that the spin-wave stiffness must be zero in paramagnets.

Finally let us note that it is possible to obtain the zero temperature limit of the fluctuation formula of the rigidity Eq. (8) within a harmonic approximation as we discuss in sec. IIA7. It is very instructive to note that the vanishment of the rigidity in the unjamming transition of a class of systems with contact forces at zero temperature is due to an exact cancellation of the Born term and the non-affine correction term [48, 53].

\section{Dynamic fluctuation formula}

Now let us briefly discuss more general, dynamic fluctuation formula for the linear response to shear-strains which describes the elasticity and viscosity in a unified manner. Although our focus is put on the elasticity rather than the viscosity in the present paper, the overview will become useful in discussions. Such a unified view point has been developed also in Ref. [54 56].

Linear response against shear deformations can be seen experimentally by measurements of the linear-viscosity (See for example [57 60]). In sec. II 1 we emphasized that for arbitrary small but finite strength of perturbation $\gamma$, static linear response break down in the $N \rightarrow \infty$ limit in solids. Similarly dynamic linear response theory will break down at large enough time scales by overwhelming non-linear contributions. However, it is still possible to delay the dominance of the non-linear responses and bring it out of a given observation time (or frequency) window by choosing sufficiently small shear-strain $\gamma$ [58]. Of course one has to choose smaller $\gamma$ for larger time window.

Within the equilibrium linear response theory the shear-stress $\sigma(t)$ at time $t$ can be related to small changes of the shear-strain $\gamma\left(t^{\prime}\right)$ in the past $t \geq t^{\prime} \geq-\infty$ as [54],

$$
\delta \sigma(t)=b \gamma(t)-\beta \int_{-\infty}^{t} d t^{\prime} \frac{\partial C_{\sigma}\left(t, t^{\prime}\right)}{\partial t^{\prime}} \gamma\left(t^{\prime}\right)
$$

Here $b$ is the born term defined in Eq. (9). The correlation function $C_{\sigma}\left(t, t^{\prime}\right)$ is the shear-stress auto-correlation function defined as,

$$
C_{\sigma}\left(t, t^{\prime}\right) \equiv N\left\langle\sigma(t) \sigma\left(t^{\prime}\right)\right\rangle
$$

We show in Appendix $\mathrm{A}$ a simple derivation of Eq. (11). The 1st and 2nd term on the r.h.s. of Eq. (11) represents respectively the instantaneous rigidity, which describes the affine part of response to shear, and the non-affine correction term due to shear-stress relaxations.

By doing an integration by parts in the r. h. s. of Eq. (11) we can find an alternative expression for the linear response,

$$
\delta \sigma(t)=\left(b-N \beta\left\langle\sigma^{2}\right\rangle\right) \gamma(t)+N \beta\langle\sigma\rangle^{2} \gamma(-\infty)+\beta \int_{-\infty}^{t} d t^{\prime} C_{\sigma}\left(t, t^{\prime}\right) \dot{\gamma}\left(t^{\prime}\right)
$$

where $\dot{\gamma}(t) \equiv d \gamma\left(t^{\prime}\right) / d t^{\prime}$ is the shear-strain rate.

More specifically, let us consider two typical experimental protocols,

- Step like shear : $\gamma(t)=\gamma \theta(t)$

The stress relaxation after then step like perturbation can be found to be,

$$
\mu(t) \equiv \delta \sigma(t) / \gamma=b-\beta\left(C_{\sigma}(t, t)-C_{\sigma}(t, 0)\right) .
$$

The initial value is nothing but the Born term

$$
\mu(0)=b,
$$


and it relaxes down to the static rigidity $\mu$ defined in Eq. (요 in the large time limit,

$$
\mu=\lim _{t \rightarrow \infty} \mu(t)=b-N \beta\left(\left\langle\sigma^{2}\right\rangle-\langle\sigma\rangle^{2}\right) .
$$

Thus the remnant stress under external strain becomes finite in solids and vanishes in liquids at the level of the linear response.

Furthermore, in the case of liquids the rigidity vanishes $\mu=0$. This implies (see Eq. (16)),

$$
b=N \beta\left\langle\sigma^{2}\right\rangle .
$$

Here we used $\langle\sigma\rangle=0$ which must always hold in liquids. It is worth to mention that the relation Eq. (17) means the born term (instantaneous rigidity) and variance of the thermal fluctuation of the stress are intimately related in liquids in equilibrium. As a result, the stress relaxation Eq. (14) becomes directly proportional to the shear-stress auto-correlation function,

$$
\mu(t)=\beta C_{\sigma}(t, 0)
$$

- Constant shear-rate : $\dot{\gamma}(t)=\dot{\gamma}$

Let us consider here liquids for which the rigidity is zero $\mu=0$. Then from Eq. (13) we find,

$$
\delta \sigma / \dot{\gamma}=\eta \quad \eta \equiv \beta \int_{0}^{\infty} d \tau C_{\sigma}(\tau)
$$

where $\eta$ is the shear-viscosity. The last equation is nothing but the Green-Kubo formula for the shear-viscosity [61].

Usually we tend to think that rigidity and viscosity are very different properties. In this respect it is very interesting to note here that both the non-affine correction term of the rigidity, which appears in the 2 nd term on the r. h. $\mathrm{s}$ of Eq. (16), and the viscosity given by Eq. (19) are related to the spontaneous thermal fluctuation of the shear stress. Quite remarkably recent numerical studies of glasses have shown that the non-affine correction term is significant even in the zero temperature limit [62, 63]. (The zero temperature limit of the fluctuation formula of the rigidity Eq. (8) is given by Eq. (37)). This implies rigidity and viscosity are related in glasses.

\section{Visco-elasticity}

Supercooled liquids are visco-elastic materials because of the two-step relaxation: the shear-stress auto-correlation function $C_{\sigma}(\tau)$ or equivalently the stress-relaxation $\mu(\tau)$ (see Eq. (18)) exhibit plateau like behaviours as shown schematically in Fig. 2 ,

The stress relaxation function $\mu(\tau)$ starts from the instantaneous rigidity, i. e. the born term $b$ at time $\tau=0$ (see Eq. (15)). Very at the beginning it exhibits a rapid decay as indicated in Fig. 2, which we call as 'initial relaxation'. The duration of the initial relaxation is expected to be of the order of the vibration time $\tau_{0}=\sqrt{m a^{2} / \epsilon}$ with $a$ being the microscopic unit length scale and $\epsilon$ being the unit energy (or collision time $\rho^{-1 / d} \sqrt{m / k_{\mathrm{B}} T}$ ). The rapid initial relaxation can be easily seen in numerical simulations 68,70$]$. Whether it lies within the time window of experiments would depend on the specific systems under study [57-60].

Then the relaxation slows down significantly exhibiting a plateau like behavior 60, 68 70], which we call as ' $\beta$ relaxation'. Let us call the height of the onset of the plateau like region as $G_{\infty}$ in the present paper. If the plateau is ideally extended for a long enough time scale, the system would appear as a solid with a finite rigidity (see Eq. (16)), which is the main concern of this paper.

The temporal solid state is characterized by the cage structure: the particles are collectively confined by the surrounding particles within a narrow space called as cage. Within the framework of the RFOT, including both the MCT [11, 12] and cloned liquid approaches [18, 20], the size of the cage $A$ is viewed as the order parameter which indicates the presence of amorphous solid state (see sec. II C 1).

Finally the $\alpha$ relaxation starts at longer time scales due to reorganization of the cages by which the residual stress $\mu(\tau)$ eventually decays down to 0 so that the viscosity $\eta$ Eq. (19) can be defined. As the temperature is lowered the relaxation time of the supercooled liquid $\tau_{\alpha}$ increases rapidly and eventually exceeds the experimental time scale. As the result the system falls out of equilibrium in experimental time scales.

Still visco-elastic properties at lower temperatures can be studied systematically by observing aging effects 34 , 71 . which allow us to explore the glassy phase below $T_{\mathrm{K}}$. For example, a typical stress-relaxation protocol [59, 60] goes 

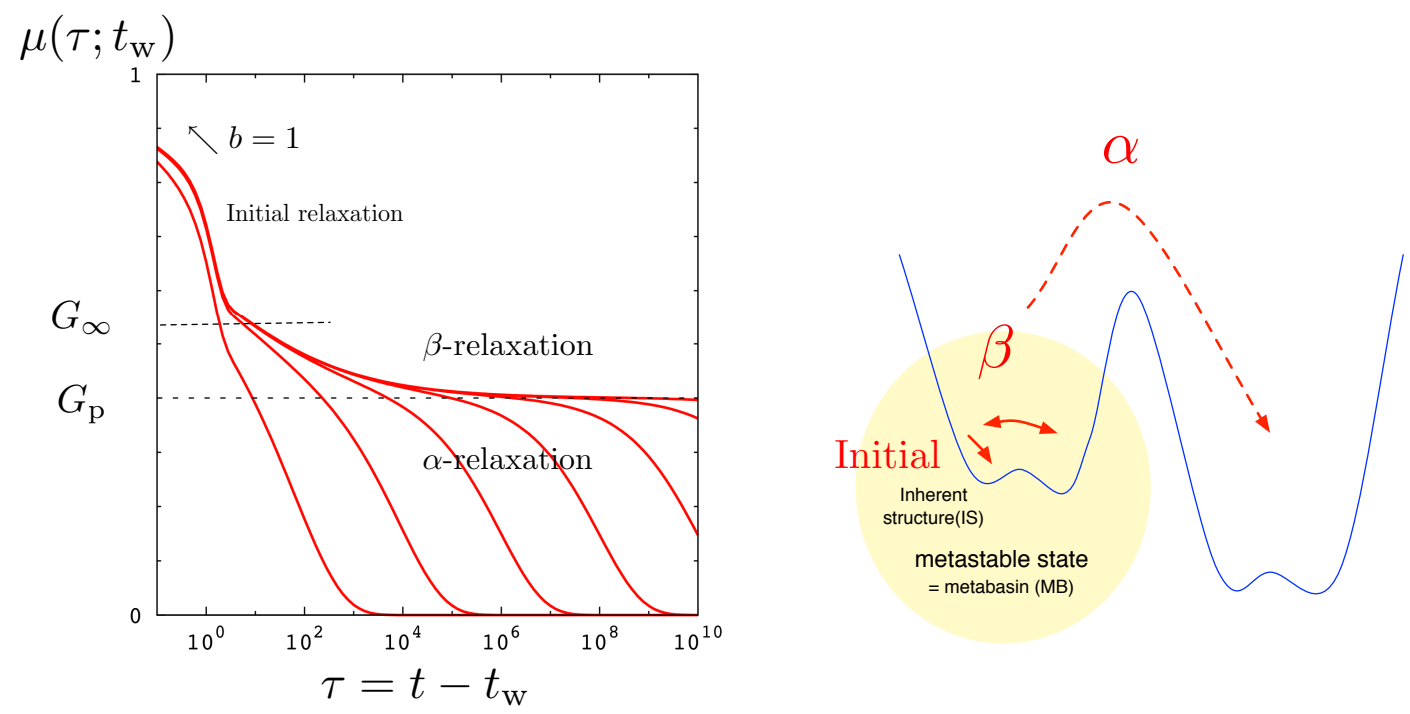

FIG. 2. Schematic, idealized picture of stress relaxation of supercooled liquid/glass under aging (left) and an intuitive freeenergy landscape picture (right). The stress relaxation function $\mu\left(\tau ; t_{\mathrm{w}}\right)$ is presented in a unit such that $\mu(0)=b=1$. Here $b$ is the Born term [35] which represents the instantaneous rigidity of system. The time $\tau$ is presented in a unit such that the microscopic time scale $\tau_{0}$, corresponding to typical vibration time of the particles, becomes 1 . The waiting time $t_{\mathrm{w}}$ increases from the left to the right curves. If the relaxation time $\tau_{\alpha}$ is finite, the curves converge to a limiting (equilibrium) curve at large enough waiting time $t_{\mathrm{w}} \gg \tau_{\alpha}$. We consider that the stress relaxation consists of three regimes. Within the initial very short time regime whose duration is just of the order of the unit time scale $\tau_{0}$, the stress relaxes rapidly starting from the instantaneous rigidity $b$ down to some value, which is often called (somewhat misleadingly) $G_{\infty}$. Most likely this is due to fast modes of purely elastic relaxation in the vicinity of inherent structures (IS) 64, 65] and that $G_{\infty}$ roughly corresponds to rigidity of inherent structures $\mu_{\mathrm{IS}}$. In the subsequent $\beta$-relaxation regime, the stress value remains within a plateau-like region in the range $G_{\mathrm{p}}<\mu(\tau)<G_{\infty}$. We argue that this relaxation takes place within metastable states, each of which is a metabasin $(\mathrm{MB})$ [66, 67] i. e. a union of ISs with large enough mutual overlaps rather than individual ISs. Furthermore we argue that $G_{\mathrm{p}}$ corresponds to rigidity of metastable states $\hat{\mu}$ which we analyze in detail in the present paper. The reduction of $\hat{\mu}$ with respect to the rigidity of inherent structures $\mu_{\mathrm{IS}}$ is attributed to thermal excitations within metastable states, such as mutually independent, localized plastic deformations. Thus the gap between $G_{\mathrm{p}}$ and $G_{\infty}$ is expected to become smaller at lower temperatures. Finally the stress relaxes down to 0 by the $\alpha$-relaxation by which the system explores larger phase space. While the $\alpha$ relaxation strongly depends on the waiting times $t_{\mathrm{w}}$, the initial and $\beta$ relaxations depend on $t_{\mathrm{w}}$ only weakly (quasi-equilibrium).

as follows: 1) the system is prepared in the equilibrium liquid state at a high enough temperature above the glass transition temperature $\left.T_{\mathrm{g}}, 2\right)$ the temperature of the bath is quenched to a target temperature $T$ below $T_{\mathrm{g}}, 3$ ) the system is left in a quiescent state for a waiting time $t_{\mathrm{w}}$ after which 4) a finite shear-strain of amplitude $\gamma$ is applied,

$$
\delta \gamma(t)=\gamma \theta\left(t-t_{\mathrm{w}}\right) .
$$

Again the stress relaxation starts from the instantaneous rigidity, the born term $b$ and exhibits the two step relaxations (+ initial relaxation) as shown in Fig. 2. But now the stress relaxation depends not only on the time $\tau$ elapsed after switching on the shear-strain but also on the waiting time $t_{\mathrm{w}}$ thus we denote it as $\mu\left(\tau ; t_{\mathrm{w}}\right)$. Most notable feature is that $\alpha$-relaxation becomes strongly dependent of the waiting time $t_{\mathrm{w}}$ while the $\beta$-relaxation only weakly depends on the waiting time $t_{\mathrm{w}}$. Then it would be meaningful to define a limiting relaxation curve for the $\beta$-regime,

$$
\mu_{\mathrm{eq}}(\tau) \equiv \lim _{t_{\mathrm{w}} \rightarrow \infty} \mu\left(\tau ; t_{\mathrm{w}}\right) .
$$

As suggested by the studies of aging effects, in particular the dynamical mean-field theory of spin-glasses [72], it is natural to expect that the fluctuation formula Eq. (18) still holds in the $\beta$-regime and that a slightly modified fluctuation formula applies for the $\alpha$-regime (See Appendix $\mathrm{A}$ ).

The system will behave as a solid with finite rigidity as long as the time scale lies within the plateau-like region. However there is an ambiguity to define such a rigidity because the plateau is not completely flat at finite time scales. As noted above we call the value at the onset of the plateau-like region as $G_{\infty}$ in the present paper. Physically it is 
more interesting to examine the value of the plateau in the asymptotic limit (See Fig. 2),

$$
G_{\mathrm{p}} \equiv \lim _{\tau \rightarrow \infty} \mu_{\mathrm{eq}}(\tau)
$$

Note that as long as the system is ergodic, which is certainly the case at high enough temperatures in the liquid phase, the limiting curve Eq. (21) covers not only the $\beta$-relaxation but also the $\alpha$-relaxation by which the shear stress relaxes down to 0 . Thus for this $G_{\mathrm{p}}$ to become non-zero, there must be ergodicity breaking, i. e. the ideal glass transition. If the putative ergodicity breaking take place at some temperature, say $T_{\mathrm{NE}}$, then the asymptotic value of the plateau at $T_{\mathrm{NE}}$ can also be obtained following the equilibrium liquid state at $T>T_{\mathrm{NE}}$ as,

$$
G_{\mathrm{p}}\left(T_{\mathrm{NE}}\right)=\lim _{\tau \rightarrow \infty} \lim _{T \rightarrow T_{\mathrm{NE}}^{+}} \mu_{\mathrm{eq}}(\tau)
$$

It is not known yet whether such a true ergodicity breaking (ideal glass transition) really exist in real systems. However even if it does not exist in the rigorous sense, we may take a pragmatic view and look for some crossover height $G_{\mathrm{p}}$ between more $\beta$-like regime and more $\alpha$-like regime.

\section{Free-energy landscape picture}

Let us discuss the visco-elasticity of supercooled liquids within the phenomenological free-energy landscape picture shown schematically in Fig. 2 [27, 64 67]. The following arguments are based on simple intuitions on some basic energetics so that they are admittedly naive in many respects and thus they should be taken with a grain of salt. Here we focus on deeply supercooled, low temperature regime. In sec. IA9 we briefly discuss the free-energy landscape at higher temperatures.

The most basic ingredients in the free-energy landscape at low temperatures are local energy minima which are called as inherent structures (IS) [64, 65]. In the phase space, each inherent structure is surrounded by a local region called as basin whose energy landscape can be described well by a purely elastic Hamiltonian (See Eq. (34) below).

A set of inherent structures and their basins may be grouped together as a metabasin (MB) [66, 67]. From the point of view of the mean-field theory which we discuss in sec. IIC] a metabasin rather than an inherent structure should be identified as a metastable state which are pure states in the mean-field theory [73]. Within the mean-field theory, metastable states are characterized by the cage size $A$ (See Eq. (61)). Two different inherent structures, say $\mathrm{IS}_{1}$ and $\mathrm{IS}_{2}$ are associated with a common metastable state if the average mean-squared distance is smaller than the cage size,

$$
\frac{1}{2 N d} \sum_{i=1}^{N}\left(\mathbf{r}_{i}^{\mathrm{IS}_{1}}-\mathbf{r}_{i}^{\mathrm{IS}_{2}}\right)^{2}<A
$$

Here $\mathbf{r}_{i}^{\mathrm{IS}_{1}}$ and $\mathbf{r}_{i}^{\mathrm{IS} \mathrm{S}_{2}}$ are configurations of the particles at the two ISs. For example consider two sets of particle configurations such that one configuration is created from the other by a localized plastic deformation involving just a few particles. Differences between the two configurations on the rest of the system will be just smooth relative elastic deformations whose amplitude decay to 0 at long distances from the region where the localized plastic deformation took place [27]. Such a pair of configurations, both of which are energy minima, can be easily generated explicitly for example by athermal quasi-static shear (AQS) processes [32, 33, 63, 74 77 ]. In the thermodynamic limit $N \rightarrow \infty$ the mean-squared distance between such a pair of states which appears on the left hand side of Eq. (24) will vanish. Although they are distinct inherent structures which are not accessible to each other by continuous elastic deformations, so that each one is out of the basin of the other, they should be regarded as belonging to the same metastable state since their thermodynamic properties must be the same. This is an extreme example but the argument can be repeated for the cases involving two, three, ... localized plastic deformations which are independent from each other.

Within a given metastable state or metabasin, there must be an inherent structure which has the lowest energy, i. e. the ground state within the metabasin. All other inherent structures are excited states in the metabasin. As the temperature increases, not only the ground state but also the excited states become more populated. Moreover different metastable states may merge together by raising the temperature. As the result the entropy of the metabsin increases with the temperature.

We consider that the stress relaxation of deeply supercooled liquids may be described within the free-energy landscape picture as follows (see Fig. 22). First, we consider that the initial rapid relaxation is mainly due to the dynamics in the basins of individual ISs, which are dominated by fast modes of the purely elastic dynamics which we discuss 
in sec. ЏA7 The subsequent slower process in the plateau-region, i.e. the $\beta$-relaxation would involve possibly two different mechanisms. One is due to slower modes of the elastic dynamics around inherent structures. The other is due to thermally activated transitions between different inherent structures in a common metabasin (metastable state). The latter is necessarily inelastic (plastic) deformations as we discuss in sec. ПA8. Finally the $\alpha$-relaxation may be attributed to thermally activated transitions between different metabasins (metastable states).

The above phenomenological picture implies thermal averaging may be factorized as,

$$
\langle\ldots\rangle=\llbracket\langle\ldots\rangle_{\mathrm{MS}} \rrbracket \quad\langle\ldots\rangle_{\mathrm{MS}} \sim\left[\langle\ldots\rangle_{\mathrm{IS}}\right]_{\mathrm{MB}}
$$

Here $\langle\ldots\rangle_{\text {MS }}$ and $\llbracket \ldots \rrbracket$ represent respectively thermal averaging within a metastable state and over different metastable states. Similarly $\langle\ldots\rangle_{\mathrm{IS}}$ and $[\ldots]_{\mathrm{MB}}$ represent respectively thermal averaging in the vicinity of an inherent structure (IS) and over different ISs within a common metabasin (MB) (or metastable state). Then thermal fluctuations of a physical quantity, say $O$, could be decomposed into intra-state and inter-state fluctuations as,

$$
\left\langle O^{2}\right\rangle-\langle O\rangle^{2}=\llbracket\left\langle O^{2}\right\rangle_{\mathrm{MS}} \rrbracket-\llbracket\langle O\rangle_{\mathrm{MS}} \rrbracket^{2}=\underbrace{\llbracket\left\langle O^{2}\right\rangle_{\mathrm{MS}}-\langle O\rangle_{\mathrm{MS}}^{2} \rrbracket}_{\text {intra-state fluctuation (initial }+\beta \text { ) }}+\underbrace{\llbracket\langle O\rangle_{\mathrm{MS}}^{2} \rrbracket-\llbracket\langle O\rangle_{\mathrm{MS}} \rrbracket^{2}}_{\text {inter-state fluctuation }(\alpha)} .
$$

Quite remarkably the cloned liquid method enables disentanglement of the intra-state and inter-state fluctuations and decompose thermal fluctuations into the two parts as Eq. (26) as we discuss later in sec IIC4.

Moreover, the intra-state fluctuation itself may be decomposed into thermal fluctuations around inherent structures and those between different inherent structures in a common metabasin,

$$
\left\langle O^{2}\right\rangle_{\mathrm{MS}}-\langle O\rangle_{\mathrm{MS}}^{2}=\left[\left\langle O^{2}\right\rangle_{\mathrm{IS}}\right]_{\mathrm{MB}}-\left[\langle O\rangle_{\mathrm{IS}}\right]_{\mathrm{MB}}^{2}=\underbrace{\left[\left\langle O^{2}\right\rangle_{\mathrm{IS}}-\langle O\rangle_{\mathrm{IS}}^{2}\right]_{\mathrm{MB}}}_{\text {fluctuation around IS (initial) }}+\underbrace{\left[\langle O\rangle_{\mathrm{IS}}^{2}\right]_{\mathrm{MB}}-\left[\langle O\rangle_{\mathrm{IS}}\right]_{\mathrm{MB}}^{2}}_{\text {inter-IS fluctuation }(\beta)}
$$

Let us note that $\beta$-relaxation may not be entirely due to the thermal activation between different inherent structures but partly due to slower modes of the elastic fluctuations around inherent structures as discussed above. How the two different relaxations mechanism are mixed in the dynamics is not clear at the moment.

\section{Hierarchy of rigidities}

The hierarchy of the thermal fluctuations naturally implies hierarchy of rigidities. The rigidity as defined by the static fluctuation formula Eq. (8) (or Eq. (16))) contains non-affine corrections represented by the correlation function of the shear-stress fluctuations. By decomposing the latter into several pieces following the above prescription we find,

$$
\mu=\underbrace{\llbracket\left[b-N \beta\left(\left\langle\sigma^{2}\right\rangle_{\mathrm{IS}}-\langle\sigma\rangle_{\mathrm{IS}}^{2}\right)\right]_{\mathrm{MB}}}_{\hat{\mu}}-N \beta\left(\left[\langle\sigma\rangle_{\mathrm{IS}}^{2}\right]_{\mathrm{MB}}-\left[\langle\sigma\rangle_{\mathrm{IS}}\right]_{\mathrm{MB}}^{2}\right)]]-N \beta\left(\llbracket\langle\sigma\rangle_{\mathrm{MS}}^{2} \rrbracket-\llbracket\langle\sigma\rangle_{\mathrm{MS}} \rrbracket^{2}\right)
$$

where we introduced the rigidity of inherent structures $\mu_{\mathrm{IS}}$,

$$
\mu_{\mathrm{IS}} \equiv b-N \beta\left(\left\langle\sigma^{2}\right\rangle_{\mathrm{IS}}-\langle\sigma\rangle_{\mathrm{IS}}^{2}\right)
$$

and the rigidity of metabasin or intra-state rigidity $\hat{\mu}$,

$$
\hat{\mu} \equiv b-N \beta\left(\left\langle\sigma^{2}\right\rangle_{\mathrm{MS}}-\langle\sigma\rangle_{\mathrm{MS}}^{2}\right)
$$

with $b$ being the Born term Eq. (9).

We speculate that the rigidity of inherent structures $\mu_{\text {IS }}$ and that of metastable states (metabasins) $\hat{\mu}$ corresponds respectively to the upper and lower bound of the plateau region ( $\beta$-relaxation) of the shear-stress relaxation (see Fig. (2),

$$
\begin{aligned}
G_{\infty} & \simeq \mu_{\mathrm{IS}}=N \beta\langle\sigma\rangle_{\mathrm{IS}}^{2} \\
G_{\mathrm{p}} & \simeq \hat{\mu}=N \beta\langle\sigma\rangle_{\mathrm{MS}}^{2}
\end{aligned}
$$

Since the initial and $\beta$-relaxations weakly depend on whether the system is in equilibrium or out-of equilibrium, we expect that the quasi-static evaluations of $G_{\infty}$ and $G_{\mathrm{p}}$ are valid for the supercooled liquid in equilibrium as well as glasses out-of equilibrium. 
The last equations of Eq. (31) and Eq. (32) relates the rigidity at different levels to the variance of the shear-stress fluctuations at the corresponding levels. It is easy to see that they must hold in liquids. First note that the total rigidity must vanish $\mu=0$ in liquids. Then note that the stress must vanish after fully taking the thermal average $\langle\sigma\rangle=\llbracket\langle\sigma\rangle_{\mathrm{MS}} \rrbracket=0$. Using these two in the expression Eq. (28) one immediately finds the last equations of Eq. (31) and Eq. (32). Note also an analogous relation Eq. (17) which reads

$$
b=\mu(t=0)=N \beta\left\langle\sigma^{2}\right\rangle .
$$

It relates the born term $b$ (instantaneous rigidity) to the variance of the total thermal fluctuation of the stress $\left\langle\sigma^{2}\right\rangle$.

\section{Rigidity of inherent structures}

Here let us discuss more explicitly the rigidity $\mu_{\mathrm{IS}}$ of inherent structures (IS). An IS [64] is a configuration of the particles in an energy minimum around which the particles make purely elastic fluctuations. In the close vicinity of an IS with the particle configuration $\left\{\left(\mathbf{r}_{\mathrm{IS}}\right)_{i}\right\}(i=1,2, \ldots, N)$, the potential part of the Hamiltonian Eq. (4) is approximated as,

$$
U=U\left(\left\{\left(x_{i}^{\mu}\right)_{\mathrm{IS}}\right\}\right)+U_{\mathrm{el}}\left(\left\{\left(u_{i}^{\mu}\right)_{\mathrm{IS}}\right\}\right)+\text { unharmonic corrections. }
$$

Here $U_{\mathrm{el}}\left(\left\{\left(u_{i}^{\mu}\right)_{\mathrm{IS}}\right\}\right)$ is the harmonic part of the energy,

$$
U_{\mathrm{el}}\left(\left\{\left(u_{i}^{\mu}\right)_{\mathrm{IS}}\right\}\right) \equiv \frac{1}{2} \sum_{i, j} \sum_{\mu \nu} H_{i j}^{\mu \nu} u_{i}^{\mu} u_{j}^{\nu} \quad u_{i}^{\mu} \equiv x_{i}^{\mu}-\left(x_{\mathrm{IS}}\right)_{i}^{\mu}
$$

with the matrix $H$ being the Hessian matrix,

$$
H_{i j}^{\mu \nu}=\delta_{\mu \nu} \delta_{i j} \sum_{k} v^{\mu \mu}\left(r_{i, k}\right)-\left.v^{\mu \nu}\left(r_{i, j}\right) \quad v^{\mu \nu}\left(r_{i, j}\right) \equiv \frac{\partial^{2} v\left(r_{i j}\right)}{\partial x_{i}^{\mu} \partial x_{j}^{\mu}}\right|_{\text {IS }}
$$

where..$\left.\right|_{\text {IS }}$ means to take derivatives at the IS, namely $x_{i}^{\mu}=\left(x_{\mathrm{IS}}\right)_{i}^{\mu}$. Then the intra-IS rigidity Eq. (29) can be expressed fully microscopically [63, 75, 78] as,

$$
\mu_{\mathrm{IS}}=b-\frac{1}{N} \sum_{i=1}^{N} \boldsymbol{\Xi}_{i} \cdot\left(\mathcal{H}^{-1} \boldsymbol{\Xi}\right)_{i}
$$

where $\mathcal{H}^{-1}$ is the inverse of the Hessian matrix Eq.

$$
\Xi_{i}=\left.\nabla_{i} \sigma\right|_{\text {IS }}
$$

with $\sigma$ being the shear-stress Eq. (7) and $\nabla_{i}=\left(\partial / \partial x_{i}, \partial / \partial y_{i}, \partial / \partial z_{i}, \ldots\right)$. More precisely, the born term Eq. (9) at the IS can be written as,

$$
b=\frac{1}{N} \sum_{i j} H_{i j}^{x x}\left(z_{\mathrm{IS}}\right)_{i}\left(z_{\mathrm{IS}}\right)_{j}
$$

and the $\boldsymbol{\Xi}$ at the IS can be written as,

$$
\Xi_{i}^{\mu}=\sum_{k} H_{i k}^{\mu x}\left(z_{\mathrm{IS}}\right)_{k} \quad(\mu=x, y, z) .
$$

Within the harmonic approximation to the potential Eq. (34), the fluctuation formula of the rigidity at finite temperatures Eq. (8) reduces exactly to Eq. (37] [78]. Non-harmonic corrections which can be treated perturbatively amount at most to $O(T)$ terms which vanish in the limit $T \rightarrow 0$.

It is instructive to rewrite the fluctuation formula Eq. (37) in terms of the eigen modes of the Hessian matrix as [33, 63, 75],

$$
\mu_{\mathrm{IS}}=b-\frac{1}{N} \sum_{\lambda} \frac{\Xi_{\lambda}^{2}}{\lambda}
$$


Here $\lambda$ 's are the eigen values of the Hessian matrix which satisfy the equations,

$$
\sum_{(j, \nu)} H_{i j}^{\mu \nu} U_{(j, \nu), \lambda}=\lambda U_{(i, \mu), \lambda}
$$

with $U_{(i, \mu), \lambda}$ being the normalized eigen vectors. The eigen values $\lambda$ of the Hessian matrix at the inherent structures (energy minima) are not negative by definition. In Eq. (41) we also introduced the projection of the $\Xi$ field onto the eigen modes,

$$
\Xi_{\lambda}=\sum_{(i, \mu)}\left(U^{\dagger}\right)_{\lambda,(i, \mu)} \Xi_{i}^{\mu}
$$

The shear-stress relaxation $\mu(t)$ can be studied easily within the harmonic approximation. For simplicity we assume the equations of motion with viscous damping,

$$
m \frac{d^{2} u_{i}^{\mu}}{d t^{2}}+m \eta \frac{d u_{i}^{\mu}}{d t}=-\sum_{(j, \nu)} H_{i j}^{\mu \nu} u_{j}^{\nu}
$$

with $m$ and $\eta$ being the mass of a particle and shear-viscosity. The coupled equations of motions can be transformed to independent equations of motions for the eigen modes $u_{\lambda} \equiv \sum_{(i, \mu)}\left(U^{\dagger}\right)_{\lambda,(i, \mu)} u_{i}^{\mu}$,

$$
\frac{d^{2} u_{\lambda}}{d t^{2}}+\eta \frac{d u_{\lambda}}{d t}=-\omega^{2}(\lambda) u_{\lambda}
$$

where we introduced characteristic frequencies $\omega$ defined as,

$$
\omega(\lambda) \equiv \sqrt{\lambda / m}
$$

We further simplify the problem considering the over-damped limit neglecting the inertial term. Then the shear-stress relaxation $\mu(t)$ after the step like perturbation is obtained as,

$$
\mu(t)=b-\int_{0}^{\infty} d \omega D(\omega) \frac{\Xi_{\omega}^{2}}{m \omega^{2}}\left[1-e^{-\left(\omega^{2} / \eta\right) t}\right] .
$$

where we introduced the distribution function $D(\omega)$ of the characteristic frequencies $\omega$,

$$
D(\omega) \equiv \frac{1}{N} \sum_{\lambda} \delta(\omega-\sqrt{\lambda / m}) .
$$

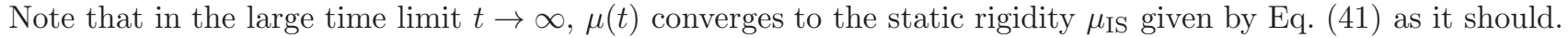

The spectrum of eigen values of the Hessian matrix of the ISs is known to constitute a continuous band extending down to $\omega=0$ so that the stress relaxation $\mu(t)$ cannot be a simple exponential relaxation even in the harmonic approximation. However convergence of $\mu(t)$ to the asymptotic value $\mu_{\mathrm{IS}}$ would be not too slow if the integrand of Eq. (47) is not singular in the $\omega \rightarrow 0$ limit. Indeed the numerical analysis of inherent structures of a model structural glass system in 75] (See Fig. 5 of the latter reference) suggests this is the case such that the a large fraction of the whole reduction of the rigidity from $b$ to $\mu_{\text {IS }}$ would be attained within the order of the microscopic time scales. This observation is consistent with our view that the initial rapid decay of stress-relaxation (see Fig. 2) is most likely purely elastic dynamics in the close vicinity of ISs.

\section{Rigidity of metabasin}

In sharp contrast to the purely elastic deformations discussed above, the transitions between different ISs, even within a common MB (See Fig. 2), necessarily involve some inelastic (plastic) deformations [27]. It is natural to expect that they are thermally excited plastic deformations, which are mostly independent from each other. We consider such processes, together with slower modes of the elastic dynamics, contribute to the slower relaxation around the plateau, i. e. the $\beta$-relaxation [65].

Let us come back to the example of the pair of IS which we discussed at the beginning of sec. II A 5 They are very closely related to each other such that the transition between the two involves only a localized plastic deformation 


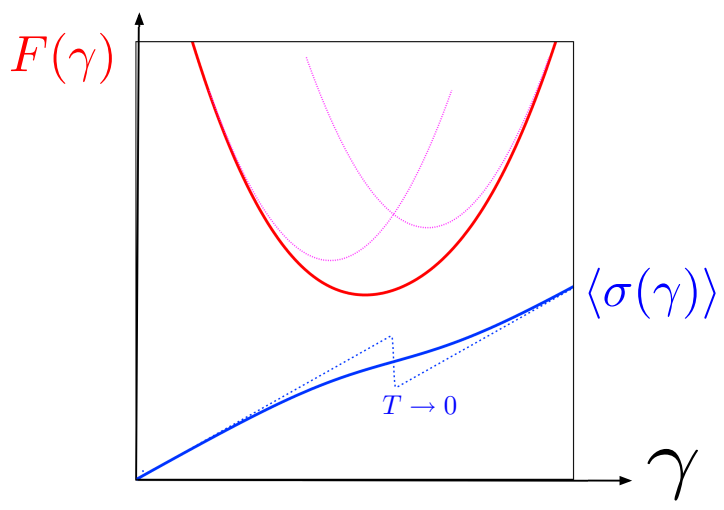

FIG. 3. Schematic free-energy landscape of metabasin under external strain and corresponding static stress-strain curve. The free-energy $F(\gamma)$ (bold line) and stress $\langle\sigma(\gamma)\rangle=d F(\gamma) / d \gamma$ (bold line) is plotted against the $\gamma$-axis. In this example two inherent structures (IS) with rigidity $\mu_{\text {IS }}$, represented by the parabolas, belong to the metabasin. At zero temperature, the level crossing between the two result in a discontinuous stress drop in the stress strain curve. At finite temperatures, the singularity at the level-crossing is removed such that the renormalized rigidity of the metabasin $\hat{\mu}$ can be defined.

involving a few particles. Such transitions between two ISs are already beyond the scope of purely harmonic theories. In such a process, typically an eigen value of the Hessian matrix becomes negative at the transition 63]. This means that elasticity as defined in the most strict sense breaks down immediately moving just a little bit away from the close vicinity of an IS.

However it is reasonable to expect that some sort of renormalized harmonic theory which takes into account the effects of some weak plasticity should exist at macroscopic level. In this respect it is instructive to recall, for example, the fact that the rigidity of spin waves of ferromagnets can still be defined in the presence of some small densities of topological defects such as pairs of vorticies (dislocations). In the latter case, thermally excited topological defects renormalizes the the rigidity down to a smaller value as the temperature is increased to higher temperatures and eventually enables melting of otherwise elastic system [52, 79].

Our picture for the rigidity of metabasin can be summarized as the following. Different ISs within a common metastable state or MB have large enough mutual overlaps in the sense of Eq. (24). They are likely to be related to each other by localized plastic deformations 27, 32, 33, 63, 74, 75, 80]. Among all ISs within a common MB there is an IS which has the lowest energy, i. .e the ground state within the MB. All other ISs in the same MB are excited states, which can acquire larger statistical weights at higher temperatures. In a sense the dynamics within a MB is "reversible" 27, 66, 67] because a transition to an excited state would be often followed by a return to the ground state. From Eq. (29) and Eq. (30) we find the intra-state rigidity $\hat{\mu}$ is reduced with respect to the rigidity of IS $\mu_{\text {IS }}$ due to the stress fluctuations between different ISs in the common MB,

$$
\hat{\mu}=\mu_{\mathrm{IS}}-N \beta\left(\left\langle\langle\sigma\rangle_{\mathrm{IS}}^{2}\right\rangle_{\mathrm{MB}}-\left\langle\langle\sigma\rangle_{\mathrm{IS}}\right\rangle_{\mathrm{MB}}^{2}\right) .
$$

In our interpretation, the non-affine correction which appears on the r. h. s. is plastic in sharp contrast to the elastic non-affine correction in the basins of inherent structures discussed in sec. IIA7. In the low temperature limit, however, the thermal excitations will be suppressed so that it is natural to expect that the two rigidities become the same,

$$
\lim _{T \rightarrow 0} \hat{\mu}=\lim _{T \rightarrow 0} \mu_{\mathrm{IS}} .
$$

In order to illustrate our picture in an alternative way, we show an intuitive free-energy landscape picture of a metabasin plotted along the axis of external strain $\gamma$ in Fig. 3 together with the corresponding static stress-strain curve. Local energy landscape of the basins around each inherent structure should be described well as a parabola with curvature $\mu_{\mathrm{IS}}$. The positions of the bottoms of the parabolas of different ISs would be slightly displaced from each other. At a given $\gamma$, the ISs can ranked according to their energy: ground state, 1st excited state, e.t.c. Along 
the $\gamma$-axis there can be level crossings among the ISs, i. e. plastic deformations. At zero temperature each level crossing would be reflected on the stress-strain curve as a stress-drop. However at finite temperatures, the free-energy landscape and the corresponding stress-strain curve should be rounded. Then it is natural to expect that the freeenergy landscape of the metabasin as a whole may be described well as a large parabola with curvature $\hat{\mu}$ which is reduced with respect to $\mu_{\mathrm{IS}}$ at finite temperatures.

\section{Free-energy landscape at higher temperature}

Let us finally turn to discuss on the free-energy landscape at higher temperatures which will provide us useful insights on how glasses melt. Within the RFOT [5 -9], the critical behaviours predicted by the mode coupling theory (MCT) [1, 12] are considered as reflections of melting or emergence of amorphous metastable solid states. If this is the case there must be an intimate analogy between the MCT criticality and the spinodal criticality of superheated crystals. In sec. IB we will discuss implication of this point of view on the rigidity problem.

It is important to note however that, at variance to the spinodal criticality of superheated crystals, the MCT criticality is relevant not only in the low temperature side but also in the high temperature side above $T_{\mathrm{c}}$. Actually MCT itself found the criticality in the higher temperature side. This point is very important in supercooled liquids which exhibit the two-step relaxations as the one shown in Fig. 2 not only at temperatures lower than $T_{\mathrm{c}}$ but also at higher temperatures around $T_{\mathrm{c}}$.

The key notion to describe the free-energy landscape of supercooled liquids at $T \rightarrow T_{\mathrm{c}}^{+}$, corresponding the inherent structures for the lower temperature side, are saddles. This has been substantiated by extensive studies of a class of solvable mean-field spin-glass models and realistic model glass forming systems [8, 43, 81]. The basic result goes as follows. The dynamics of a super-cooled liquid at $T \rightarrow T_{\mathrm{c}}^{+}$can be viewed as a point in the phase space going through a narrow channel along which the energy can be decreased. As the time goes on, the number of eigen modes of the temporal Hessian matrix with negative eigen values becomes less and less. In the large time limit the system ends up in a marginally stable state: all the eigen values of the temporal Hessian matrix turns out to be positive but the lowest one is touching zero. According to this picture, the main mechanism of the slow dynamics as $T \rightarrow T_{\mathrm{c}}^{+}$is a sort of critical dynamics distinct from the activated dynamics which becomes relevant at lower temperatures.

The essential difference between saddles and inherent structures are due to the slow modes with smaller eigen values. The saddles which are relevant for the long time dynamics as $T \rightarrow T_{\mathrm{c}}^{+}$contain a large number of positive eigen values and a small number of slightly negative eigen values. Then it is tempting to speculate that a somewhat similar elastic description of the stress relaxation as expressed by Eq. (47) would be also possible for $T>T_{\mathrm{c}}$ for short enough time scales.

Conversely if a supercooled liquid initially at a temperature below $T_{\mathrm{c}}$ is heated up to a temperature above $T_{\mathrm{c}}$, the temporal Hessian matrix will acquire a fraction of negative eigen-values. Even with one negative eigen value, the system will yield by arbitrary weak shear perturbations leading to collapse of a metastable amorphous solid state. This suggests that the static rigidity of a (temporal) metastable amorphous state would vanish approaching $T_{\mathrm{c}}$ from below.

\section{B. Connection between the order parameter and rigidity : mean-field points of view}

As we discussed in sec IA 1 emergence of rigidity $\mu>0$ in solids means break down of the commutation of the two limits, namely the thermodynamic $N \rightarrow \infty$ and small shear-strain limit $\gamma \rightarrow 0$. Thus the rigidity detects the liquid-solid phase transition as sensitively as the order parameters. Here we show that several mean-field theories generically suggest an explicit connection between the rigidity and the order parameter.

In the introduction of the present paper we mentioned the Born's rigidity crisis scenario [37] which says that melting of solids may be signaled by vanishing of the rigidity $\mu$. The problem is how it vanishes: continuously or discontinuously? Usually Born's conjecture is interpreted as suggesting continuous vanishment. This is an interesting and much debated question in the case of superheated crystals 38 41] approaching their metastability limit at $T_{\mathrm{S}}$ [82]. The same question is relevant also for supercooled liquids and glasses since, as emphasized in a recent work [42], the dynamical transition at $T_{\mathrm{d}}$ (or the MCT critical temperature $T_{\mathrm{c}}$ ) can be regarded as an analogue of the spinodal behaviour of superheated crystals at $T_{\mathrm{s}}$. We show below that some mean-field theories indeed suggest a common, universal behaviour of the rigidity approaching the metastability limits of solids including both the crystalline and amorphous systems.

In the following we first develop a simple mean-field description of the rigidity of a superheated crystalline system (ferromagnet). Then we recall the predictions by the MCT on the fate of the rigidity of amorphous solids. 


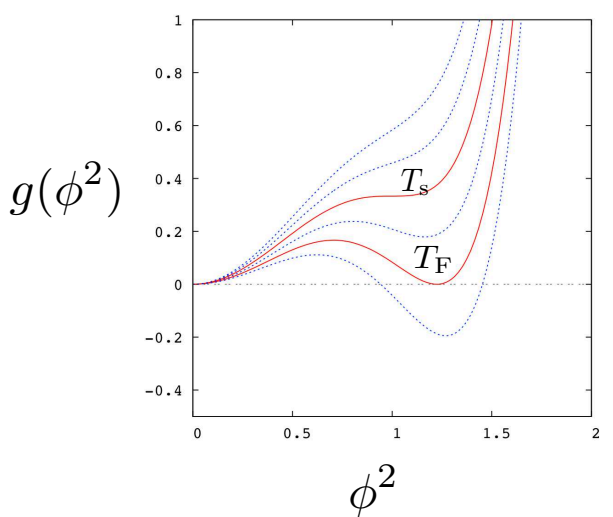

FIG. 4. A model Landau potential. The potential is defined in Eq. (51). The temperatures are $T=5 / 8,6 / 8, \ldots, 10 / 8$ from the bottom to the top curves. The curves at the spinodal temperature $T_{\mathrm{s}}=1$ and the 1 st order phase transition $T_{\mathrm{F}}=3 / 4$ are indicated. The two minima on the left and right corresponds respectively to the paramagnetic and ferromagnetic state. The equivalent effective potential of structural glasses and related spin-glass systems is the so called Franz-Parisi potential [83] for which the horizontal axis should be interpreted as the axis for the Edwards-Anderson order parameter $q_{\mathrm{EA}}$ or the cage size $A$. In the latter case the two minima on the left and right corresponds respectively to the paramagnetic state $q=0$ (liquid $A=\infty$ ) and spin-glass state $q>0$ (glass $A<\infty$ ). The two temperatures $T_{\mathrm{s}}$ and $T_{\mathrm{F}}$ corresponds respectively to the dynamical transition temperature $T_{\mathrm{d}}$ (or the MCT transition temperature $T_{\mathrm{c}}$ ) and the Kauzmann transition temperature $T_{\mathrm{K}}$.

\section{Rigidity in a GL theory for superheating}

Let us first discuss the fate of the rigidity of superheated crystalline systems approaching their metastability limits. For simplicity we consider Ginzburg-Landau (GL) theory for a vectorial spin system which can realize superheated, metastable ferromagnetic phase. By slightly generalizing the usual scalar GL theory for superheating [82], we consider an $O(2)$ symmetric $\phi^{6}$ theory described by a free-energy functional,

$$
F[\vec{\phi}]=\int d^{d} r\left[\frac{c}{2}(\nabla \vec{\phi})^{2}+g\left(|\vec{\phi}|^{2}\right)\right] \quad g(y)=T y-y^{2}+\frac{y^{3}}{3}
$$

where $\vec{\phi}(\mathbf{r})=\left(\phi_{1}(\mathbf{r}), \phi_{2}(\mathbf{r})\right)$ is a continuous vectorial field in a $d$-dimensional space. The profile of the Landau potential $g\left(\phi^{2}\right)$ is shown in Fig. 目.

Let us first examine the equilibrium states. The equilibrium states are uniform in space and parametrized by the amplitude of the order parameter $M=|\phi|=\sqrt{\phi_{1}^{2}+\phi_{2}^{2}}$. At temperatures below the spinodal temperature $T_{\mathrm{s}}=1$, there is a metastable ferromagnetic state with non-zero amplitude of the order parameter $M>0$. Equilibrium 1st order phase transition from the paramagnetic state $M=0$ to the ferromagnetic state $M>0$ takes place at a temperature $T_{\mathrm{F}}=3 / 4$ lower than the spinodal temperature $T_{\mathrm{s}}=1$.

Upon heating the metastable ferromagnet above $T_{\mathrm{F}}$ up to $T_{\mathrm{s}}$, the amplitude of the order parameter $M$ exhibits a discontinuous jump from a finite value $M>0$ (metastable ferromagnetic state) to zero $M=0$ (paramagnetic state ) signaled by a preceding square-root singularity,

$$
M(T)-M\left(T_{\mathrm{s}}\right) \propto \sqrt{T_{\mathrm{s}}-T} .
$$

The mixed character of 1st and 2nd order phase transitions is the generic feature of the spinodal criticality [82]: the order parameter vanishes discontinuously much as usual 1st order equilibrium melting transitions but the jump accompanies a preceding square-root behaviour as $T \rightarrow T_{\mathrm{s}}^{-}$.

Now let us consider spatial fluctuation of the order parameter. To this end it is convenient to re-parametrize the order parameter using 'angular variable' $\theta(\mathbf{r})$ as,

$$
\vec{\phi}(\mathbf{r})=|\vec{\phi}|(\mathbf{r})(\cos \theta(\mathbf{r}), \sin \theta(\mathbf{r})) .
$$

Then the free-energy functional Eq. (51) can be rewritten as,

$$
F[\mid \vec{\phi} \|, \theta]=\int d^{d} r\left[\frac{\mu}{2}(\nabla \theta)^{2}+\frac{c}{2}(\nabla|\vec{\phi}|)^{2}+g\left(|\vec{\phi}|^{2}\right)\right]
$$


with

$$
\mu=c|\vec{\phi}|^{2} .
$$

Note that the last two terms of the integrand in Eq. (54) appear also in the usual scalar GL theory. On the other hand, the 1st term of the integrand can be naturally interpreted as the 'spin-wave' term.

An important observation is that the rigidity $\mu$ of spin-waves is simply proportional to the square of the order parameter at this mean-field level [52]. In particular, assuming that the amplitude of the order parameter is uniform in space and takes the equilibrium value $M(T)$ we find,

$$
\mu(T)-\mu\left(T_{\mathrm{s}}\right) \propto c \sqrt{T_{\mathrm{s}}-T} .
$$

using Eq. (52) in Eq. (55). Thus at the pure mean-field level the rigidity of the spin-wave $\mu$ also exhibits a discontinuous behavior preceded by a square-root singularity as $T \rightarrow T_{\mathrm{s}}^{-}$.

Note that the situation is quite different from the case of the usual second order ferromagnetic transition (e. $g$. $\phi^{4}$ theory) [52]. In the latter case the amplitude of the order parameter exhibit a similar square-root behaviour $M(T) \propto \sqrt{T_{\mathrm{c}}-T}$ approaching the critical temperature $T_{\mathrm{c}}$ from below but with no discontinuity at $T_{\mathrm{c}}$. Then using the latter in Eq. (55) one finds that the spin-wave stiffness scales as $\mu(T) \propto c\left(T_{\mathrm{c}}-T\right)$.

\section{Mode coupling theory}

The mode coupling theory $(\mathrm{MCT})$ [11, 12] predicts that the density auto-correlation function $C_{\rho}(\tau)$, such as the self-intermediate scattering function, exhibits a two-step relaxation with a plateau similar to the one shown in Fig. 2 in supercooled liquids

and that the ergodocity breaking takes place at the MCT critical temperature $T_{\mathrm{c}}$ : the $\alpha$-relaxation time $\tau_{\alpha}$ diverges as $T \rightarrow T_{\mathrm{c}}^{+}$. Physically this means emergence of amorphous solid states with the cage structure. The amplitude of the plateau of the density auto-correlation function $C_{\rho}(\tau)$ is related to the cage size $A$ which is the order parameter within the RFOT (see Eq. (60) ). The MCT predicts that the cage size $A$ exhibits a discontinuous jump from 0 to a finite value as $T \rightarrow T_{\mathrm{c}}^{+}$followed by a square-root behaviour for $T<T_{\mathrm{c}}$,

$$
A\left(T_{\mathrm{c}}\right)-A(T) \propto \sqrt{T_{\mathrm{c}}-T} .
$$

Note that the behaviour is very similar to that of the order parameter of the superheated crystalline system Eq. (52).

The cage size $A$ can also be defined in a static matter (see Eq. (61)). In the cloned liquid approach [18 20], it is obtained by extremizing an effective free-energy which is a function of $A$ (see sec IC5). The effective free-energy corresponds to the so called Franz-Parisi potential [83] which has essentially the same qualitative features as the Landau potential shown in Fig. 4. The cloned liquid approach [20] predicts that the minimum of the potential corresponding to the metastable amorphous solid state $A<\infty$ disappears approaching the dynamical transition temperature $T_{\mathrm{d}}$ from below, i. e. as $T \rightarrow T_{\mathrm{d}}^{-}$. The dynamical transition temperature $T_{\mathrm{d}}$ is considered to corresponds to the MCT critical temperature $T_{\mathrm{c}}$. The resultant behaviour of the cage size $A$ is the same as that predicted by the MCT Eq. (57). Quantitative comparisons of the results by the dynamic and static approaches are currently undertaken intensively [22].

Within the convectional MCT, the behavior of the the stress-autocorrrelation function $C_{\sigma}(\tau)$ is obtained by projecting it onto the density auto-correlation function $C_{\rho}(\tau)[68,84]$. The former is assumed to be simply proportional to the square of the latter $C_{\sigma}(\tau) \propto C_{\rho}(\tau)^{2}$ which follows from the so called factorization approximation [84]. As the result the MCT predicts a similar plateau behaviour for the stress relaxation function $\mu(\tau)$ as for the density auto-correlation function $C_{\rho}(\tau)$ : the asymptotic plateau value of the the stress-relaxation curve $\mu(\tau)=\beta C_{\sigma}(\tau)$ (see Eq. (18)) exhibits a discontinuous jump from 0 to a finite value $G_{\mathrm{p}}\left(T_{\mathrm{c}}\right)$ as $T \rightarrow T_{\mathrm{c}}^{+}$followed by a square-root behaviour for $T<T_{\mathrm{c}}$,

$$
G_{\mathrm{p}}\left(T_{\mathrm{c}}\right)-G_{\mathrm{p}}(T) \propto \sqrt{T_{\mathrm{c}}-T} .
$$

Note that this is again very similar to the behaviour of the rigidity of the superheated crystalline system Eq. (566).

\section{Discussion}

The discussions presented above suggest common features of the rigidity of crystalline and amorphous solids at the mean-field level. First of all, the rigidity does look like the order parameter: it is just the square of the order 
parameter. As the result, it vanishes discontinuously preceded by a square-root behaviour approaching the spinodal like melting temperature from below. Although the preceding square-root singularity somehow signals the melting, the discontinuous jump of the rigidity disagrees with the usual interpretations of the Born's original rigidity crisis scenario 37 40].

The phenomenological GL approach like the one presented in sec II 1 is simple and instructive by itself. The local mean-field (Landau) potential $g\left(\phi^{2}\right)$ (see Fig. 4) has an analogous one for glasses, namely the Franz-Parisi potential [83] as we mentioned above. Indeed we demonstrate in Appendix $\mathrm{C}$ that this approach can be naturally extended to the case of vectorial spin-glass system using some replica field theoretic formalism, which implies again the same generic behaviour at $T_{\mathrm{d}}$ : discontinuous spin-wave stiffness preceded by a square-root singularity.

However in the main part of the present paper (sec III), we will analyze the static rigidity of glasses starting from a microscopic level using the fluctuation formula of the shear-modulus (see sec IA2) rather than working with the phenomenological GL like approach. As the result we will obtain the rigidity of the metastable amorphous solid states, i. e. the intra-state rigidity $\hat{\mu}$ Eq. (30) in power series of the cage size $A$ as Eq. (156) which reads,

$$
\hat{\mu}(A)=c_{0}-c_{1} A+O\left(A^{2}\right) .
$$

with $c_{0}$ and $c_{1}$ are positive constants which we evaluated explicitly. Thus we do find an intimate connection between the rigidity and the order parameter. Based on the result, we will come back to the question on the fate the rigidity in sec. IIIE.

Finally, we should remind ourselves after all that the spinodal like criticality only exists at the pure mean-field level and that to what extent the signatures of such a criticality remains in realistic systems is an open question [82].

\section{Cloned liquid theory}

\section{Cage size: order parameter}

The basic picture of the cloned liquid theory [18 20] is to view the amorphous solid state such that each particle is frozen in a disordered configuration which looks exactly like a liquid but allowed to fluctuate around the mean position within a finite range called as the cage size $A$. The cage size $A$ is the order parameter of the theory which is determined self-consistently by extremizing a free-energy function $G(A)$. The order parameter distinguishes between the liquid state $A=\infty$ and the solid state $A<\infty$. Such an idea has been proposed originally in the context of density functional theory for amorphous solids [85 88].

An important feature of the order parameter $A$ is that it can be viewed both dynamically and statically [87, 88] much as the Edwards-Anderson order parameter of spin-glasses [89]. First suppose that we monitor the dynamics of the system with the Hamiltonian Eq. (44) keeping track of the positions of the particles $\mathbf{r}_{i}(t)(i=1,2, \ldots, N)$ as a function of time $t$. Then measuring the mean-squared displacement (MSD) of the particles, we may detect existence a metastable state if the MSD tends to saturate to a finite value in a large time limit,

$$
A_{\mathrm{d}} \equiv \lim _{t \rightarrow \infty} \frac{1}{2 N d} \sum_{i=1}^{N}\left\langle\left(\mathbf{r}_{i}(t)-\mathbf{r}_{i}(0)\right)^{2}\right\rangle_{\mathrm{ini}} .
$$

Here $\langle\ldots\rangle_{\text {ini }}$ is the average over diffident initial conditions. Indeed the mode coupling theory (MCT) [11, 12] has detected a transition from the liquid phase $A_{\mathrm{d}}=\infty$ to a metastable solid phase $A_{\mathrm{d}}<\infty$ at the MCT critical temperature $T_{\mathrm{c}}$. Alternatively the same cage size can also be obtained in a static way using two replicas, say $a$ and $b$, which have no mutual interactions but obey exactly the same Hamiltonian Eq. (4),

$$
A_{\mathrm{d}}=A \equiv \frac{1}{2 N d} \sum_{i=1}^{N}\left\langle\left(\mathbf{r}_{i}^{a}-\mathbf{r}_{i}^{b}\right)^{2}\right\rangle
$$

where $\mathbf{r}_{i}^{a}(t)$ and $\mathbf{r}_{i}^{b}(t)$ are particle configuration of the replica $a$ and $b$ respectively.

\section{Mean-field scenario}

At the mean field level, the RFOT theory [5 7, 9] assumes the following idealized features. First we assume that there are metastable states $\alpha=1,2, \ldots$ with free-energy $N f_{\alpha}$. The metastable states are characterized by the cage size $A$ discussed above: 1 ) if we prepare the system in any one of the metastable states, the system remains there 
such that $A_{\mathrm{d}}$ as defined in Eq. (60) is finite and 2) if we prepare two replicas in that metastable state, they remain there such that $A_{\mathrm{s}}$ as defined in Eq. (61) is finite which takes the same value as $A_{\mathrm{d}}$. Then the total free-energy of the supercooled liquid state and the ideal glass state can be written formally as,

$$
-\beta F(T)=\log Z \quad Z=\sum_{\alpha} e^{-\beta N f_{\alpha}}=\int d f e^{-N(\beta f-\Sigma(f, T))} .
$$

In the last equation the complexity (structural entropy) $\Sigma(f, t)$ is introduced as,

$$
N \Sigma(f, t) \equiv \log \sum_{\alpha} \delta\left(f-f_{\alpha}(T)\right) .
$$

We assume that there are exponentially large number of metastable states in the supercooled and glass phase such that the complexity is finite. Apparently this description is the same as the free-energy landscape picture based on the notion of inherent structure [64, 65]. However, as we noted in sec. [IA5, the metastable state in the context of the mean-field theory corresponds to metabasins rather than individual inherent structures.

At the dynamical transition $T_{\mathrm{d}}$, the complexity $\Sigma>0$ emerges. Usually the complexity $\Sigma(f, T)$ is a function of $f$ which is convex upward and vanishes continuously approaching some $f_{\min }(T)$ from above. (See Fig. 5 b)) The total free-energy of the system below $T_{\mathrm{d}}$ is given by the saddle point value of the free-energy $f$ which dominate the integral in the 2nd equation of Eq. (62). Similarly, the structural entropy is given by the value of the complexity at the saddle point. Upon lowering the temperature, the free-energy and the complexity at the saddle point decreases. Finally the structural entropy vanishes at the Kauzmann transition temperature $T_{\mathrm{K}}$, i. .e the entropy crisis. To summarize, apparently different groups of metastable states become thermodynamically relevant at different temperatures in the supercooled liquid state $T>T_{\mathrm{K}}$ while the equilibrium statistical weight condenses onto a certain amorphous "ground state" at $T<T_{\mathrm{K}}$.

The scenario described above is known to hold precisely in a class of mean-field spin-glass models [5, 6, 90, 92, 124] in which the states $\alpha=1,2, \ldots$ correspond to the solutions of the equation of states called as the Thouless-AndersonPalmer equations. For the structural glass, the cloned liquid approach substantiated the scenario starting from microscopic Hamiltonian as we sketch below.

\section{Cloning}

Now let us recall the idea of cloning [18, 24, 83]. We consider $m$ replicas (copies) $a=1,2, \ldots, m$ of the original system which stay in the same metastable state (thus the name 'cloning'). The free-energy of the cloned system can be written formally as,

$$
-\beta m F_{m}(T)=\log Z_{m} \quad Z_{m}=\sum_{\alpha} e^{-m \beta N f_{\alpha}(T)}=\int d f e^{-N(m \beta f-\Sigma(f, T))} .
$$

Note that there is only one summation over the states $\alpha$ instead of $m$ summations which is because we are assuming here that different replicas are staying in the same metastable state. We will recall shortly later (sec. IIC 5) how to realize such a state in practice.

Now we may regard $m$ as a variational real number. Then we can notice that the saddle point $f_{\mathrm{sp}}(T, m)$ which dominate the integral in the 2nd equation of Eq. (64) can be moved around by varying $m$ without changing the temperature $T$ itself (See Fig. 5 b)). As we increase $m$, the saddle point $f_{\mathrm{sp}}(T, m)$ becomes smaller and at some critical $m^{*}(T)$, the complexity at the saddle point Eq. (65) vanishes, just like the physical $(m=1)$ Kauzmann transition. This observation suggests that even at very low temperatures below the putative Kauzmann transition temperature $T_{\mathrm{K}}$, we may choose sufficiently small $m$ such that the cloned system as a whole still remain effectively

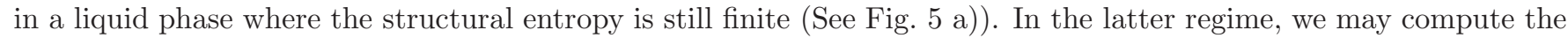
free-energy $F_{m}(T)$ of the whole system regarding it simply as a liquid using the conventional liquid theory [23] as we recall shortly later. Then the value of the complexity can be evaluated as [24],

$$
\Sigma\left(f_{\mathrm{sp}}(T, m), T\right)=\frac{1}{N} \frac{m^{2}}{T} \frac{\partial F_{m}(T)}{\partial m}
$$

The whole profile of the complexity curve $\Sigma(f, T)$ at a fixed temperature $T$ can be "scanned" (See Fig. 5 b)) by varying $m$ by which the position of the saddle point $f_{\mathrm{sp}}(T, m)$,

$$
f_{\mathrm{sp}}(T, m)=\frac{1}{N} \frac{\partial\left(m F_{m}(T)\right)}{\partial m}
$$


can be varied at will. Now we can locate the critical point $m^{*}(T)$ where the structural entropy vanishes

$$
\Sigma\left(f_{\mathrm{sp}}\left(T, m^{*}\right), T\right)=0
$$

for each temperature $T$. Further increase of $m$ does not change the saddle point value of the free-energy. This implies the value of the free-energy in the glass phase $T<T_{\mathrm{K}}$ is given by,

$$
\lim _{m \rightarrow 1^{-}} F_{m}(T)=F_{m^{*}}(T) .
$$

The Kauzmann temperature $T_{\mathrm{K}}$ is located by (See Fig. 5 b)),

$$
m^{*}\left(T_{\mathrm{K}}\right)=1
$$

At higher temperatures $T>T_{\mathrm{K}}, m^{*}=1$.

Thus the system in the glass phase $T<T_{\mathrm{K}}$ can be viewed as a liquid at an effective temperature,

$$
T^{*}=\frac{T}{m^{*}(T)}
$$

Typically it is found that $m^{*}(T) \sim T / T_{\mathrm{K}}$ so that $T^{*}=T / m^{*}(T) \sim T_{\mathrm{K}}[18,19$. This implies configuration of the system below $T_{\mathrm{K}}$ is almost like that of the liquid at $T_{\mathrm{K}}$ independently of the temperature $T$. On the other hand, in the supercooled liquid state $T>T_{K}, m^{*}(T)=1$ so that $T^{*}=T$.

a)

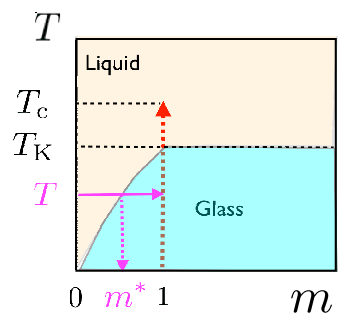

b)

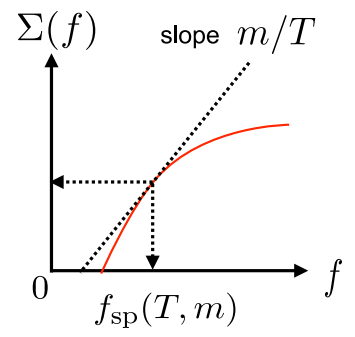

FIG. 5. Cloned liquid method: a) schematic $m-T$ phase diagram b) complexity

\section{Disentangle intra-state and inter-state responses}

Let us make here a general remark that the cloning method allows disentanglement of generic linear response functions into intra-state and inter-state responses much as anticipated by the phenomenological free-energy landscape picture discussed in sec. IIA5 (See Eq. (26)). In the present paper we fully makes use of this feature to analyze the rigidity of structural glasses. This is a strong advantage of the use of the replica method because the two responses are physically quite different in glassy systems: $\beta$ and $\alpha$ relaxations as we discussed in sec. IA4. In Appendix B we demonstrate the method works exactly in a typical mean-field spin-glass model.

Suppose that we have a physical observable of our interest, say $O$, which can be coupled to a conjugate external field $h$ so that the Hamiltonian of the system can be written as,

$$
H=H_{0}-h O,
$$

where $H_{0}$ is the original Hamiltonian of the system. Suppose that there are many metastable states $\alpha=1,2, \ldots$ such that the free-energy of the system can be written as Eq. (62), which reads,

$$
-\beta F(T, h)=\log Z \quad Z=\sum_{\alpha} e^{-N \beta f_{\alpha}(T, h)} .
$$

The static susceptibility,

$$
\left.\chi \equiv \frac{1}{N} \frac{\partial\langle O\rangle}{\partial h}\right|_{h=0}=\left.\frac{1}{N} \frac{\partial^{2} F(T, h)}{\partial h^{2}}\right|_{h=0}=\frac{\beta}{N}\left(\left\langle O^{2}\right\rangle-\langle O\rangle^{2}\right)
$$


can be formally decomposed as,

$$
\chi=\hat{\chi}+\tilde{\chi}
$$

with

$$
\hat{\chi} \equiv \llbracket \chi_{\alpha} \rrbracket \quad \tilde{\chi} \equiv \frac{\beta}{N}\left(\llbracket O_{\alpha}^{2} \rrbracket-\llbracket O_{\alpha} \rrbracket^{2}\right)
$$

where we introduced averaging over different metastable states,

$$
\llbracket \ldots \rrbracket \equiv \sum_{\alpha} P_{\alpha}(h) \ldots \quad P_{\alpha}(h) \equiv \frac{e^{-N \beta f_{\alpha}(T, h)}}{Z}
$$

and defined thermal averages within metastable states,

$$
O_{\alpha} \equiv\langle O\rangle_{\alpha}=\left.N \frac{\partial f_{\alpha}(h)}{\partial h}\right|_{h=0} \quad \chi_{\alpha}=\frac{\beta}{N}\left(\left\langle O^{2}\right\rangle_{\alpha}-\langle O\rangle_{\alpha}^{2}\right)=\left.\frac{\partial^{2} f_{\alpha}(h)}{\partial h^{2}}\right|_{h=0}
$$

Here $\langle\ldots\rangle_{\alpha}$ stands for a thermal average with respect to thermal fluctuations within the metastable state $\alpha$. By inspecting the 1st and 2nd term in the decomposition of the susceptibility Eq. (74), namely $\hat{\chi}$ and $\tilde{\chi}$ which are defined in Eq. (75), one can realize that they can be naturally interpreted as intra-state and inter-state susceptibilities respectively.

Now let us turn to consider linear response of a cloned system. The Hamiltonian of the replicated system with replicas $(a=1,2, \ldots, m)$ can be written as,

$$
H_{m}=\sum_{a=1}^{m}\left[\left(H_{0}\right)^{a}-h^{a} O^{a}\right]
$$

Here we have put probing field $h^{a}$ on each replica $(a=1,2, \ldots, m)$. The free-energy of the cloned system reads,

$$
-\beta m F_{m}\left(T,\left\{h^{a}\right\}\right)=\log Z_{m}\left(T,\left\{h^{a}\right\}\right) \quad Z_{m}\left(T,\left\{h^{a}\right\}\right)=\sum_{\alpha} e^{-N \beta \sum_{a=1}^{m} f_{\alpha}\left(T, h^{a}\right)}
$$

Note that different replicas stay in the same metastable state $\alpha$ but subjected to different probing fields $h^{a}(a=$ $1,2, \ldots, m)$. Then we find a generic susceptibility of the cloned system,

$$
\left.\chi_{a b} \equiv \frac{m}{N} \frac{\partial^{2} F_{m}\left(T,\left\{h^{a}\right\}\right)}{\partial h^{a} \partial h^{b}}\right|_{\left\{h^{a}=0\right\}}=\frac{\beta}{N}\left(\left\langle O^{a} O^{b}\right\rangle-\left\langle O^{a}\right\rangle\left\langle O^{b}\right\rangle\right)=\hat{\chi}_{m} \delta_{a b}+\tilde{\chi}_{m}
$$

with

$$
\hat{\chi}_{m} \equiv \llbracket \chi_{\alpha} \rrbracket_{T, m} \quad \tilde{\chi}_{m} \equiv \frac{\beta}{N}\left(\llbracket O_{\alpha}^{2} \rrbracket_{T, m}-\llbracket O_{\alpha} \rrbracket_{T, m}^{2}\right)
$$

where

$$
\llbracket \ldots \rrbracket_{T, m} \equiv \sum_{\alpha} P_{\alpha}(h, T, m) \ldots \quad P_{\alpha}(h, T, m) \equiv \frac{e^{-N m \beta f_{\alpha}(T, h)}}{Z_{m}} \quad Z_{m}=\sum_{\alpha} e^{-N m \beta f_{\alpha}(T, h)}
$$

Here $\hat{\chi}$ and $\tilde{\chi}$ are nothing but the intra-state and inter-state susceptibilities defined in Eq. (75) but at the effective temperature $T / m$. By taking trace over the replica index we find,

$$
\sum_{b=1}^{m} \chi_{a b}=\hat{\chi}_{m}+m \tilde{\chi}_{m}
$$

In the limit $m \rightarrow 1$ this becomes nothing but the total susceptibility of the original system (see Eq. (774)),

$$
\chi=\lim _{m \rightarrow 1^{-}} \sum_{b=1}^{m} \chi_{a b}=\hat{\chi}_{m^{*}(T)}+m^{*}(T) \tilde{\chi}_{m^{*}(T)} .
$$

Here we are assuming that we evaluate the terms on the r. h. s. in the liquid state (See Eq. (68)).

Let us list below some observations and remarks, 
- In the cloned "liquid" system, the total susceptibility Eq. (84) is just that of a (molecular) liquid state at temperature $T^{*}=T / m^{*}$.

- By comparing Eq. (80) and Eq. (74) we notice that replica trick allows us to disentangle the intra-state $(\beta$ relaxation) and inter-state response ( $\alpha$-relaxation) hided in the (cloned) liquid: to find the intra-state susceptibility $\hat{\chi}$ one simply needs to look at the coefficient of the terms with $\delta_{a b}$ in the susceptibility matrix $\chi_{a b}$.

- Note that the decomposition can be done not only in the glass phase where $m=m^{*}(T)<1$ but also at higher temperatures where $m=m^{*}(T)=1$ as long as the metastable states (with finite cage size $A$ ) exist. This is made possible by introducing $m$ clones and taking $m \rightarrow 1$ limit afterwards (see Appendix B).

- In the standard interpretation of the RSB solution for the glass phase [90, 91], which is based on solvable meanfield spin-glass models ( see Appendix [B] , the factor $m^{*}(T)(\leq 1)$ ) which appears in front of $\tilde{\chi}$ in Eq. (84) is understood as the probability that two independent replicas stay at different metastable states (here the replicas are not the 'cloned' ones). In a more physical term, it roughly corresponds to the probability that a given system stay at an low lying 'excited' metastable state rather than 'ground' metastable state in the glass phase. The parameter $m^{*}(T)$ is decreasing function of the temperature $T$ and typically behaves as $m \sim T / T_{\mathrm{K}}[92$.

The idea presented above is generic but needs to be implemented more explicitly. In sec. IC 7. we present a recipe to do realize the decomposition using the cage expansion.

\section{Molecular liquid}

Now we recall a microscopic implementation of the idea of the cloned liquid [18]. The idea is to consider a molecular liquid state: a liquid with an assembly of hypothetical "molecules" $i=1,2, \ldots, N$ with the center of mass (CM) at $\mathbf{R}_{i}$, which itself consists of original particles of different replicas $a=1,2, \ldots, m$ at $\mathbf{r}_{i}^{a}$. The task goes as follows. First one put an artificial confining potential to a create molecular state: we start from a 'forced' cloned system whose free-energy Eq. (64) is given by,

$$
-\beta m F_{m}=\log Z_{m} \quad Z_{m}=\frac{1}{(N !)^{m}} \int \prod_{i=1}^{N} \prod_{a=1}^{m} \frac{d^{d} r_{i}^{a}}{\Lambda^{d}} e^{-\beta H_{m}}
$$

where the potential part of the Hamiltonian given by,

$$
H_{m}=\sum_{a=1}^{m} \sum_{i<j} v\left(r_{i j}^{a}\right)-\frac{1}{4 \alpha} \sum_{i=1}^{N} \sum_{a, b=1}^{m}\left|\mathbf{r}_{i}^{a}-\mathbf{r}_{i}^{b}\right|^{2}
$$

Here the 1st term on the r.h.s is the two-body interaction potential Eq. (4). The 2nd term is introduced to confine the particles in different replicas to develop a 'forced' molecular state. The cage size $A$ of the resultant system is defined as,

$$
\frac{m(m-1)}{2} N d A \equiv \frac{1}{4} \sum_{i=1}^{N} \sum_{a, b=1}^{m}\left\langle\left(\mathbf{r}_{i}^{a}-\mathbf{r}_{i}^{b}\right)^{2}\right\rangle=\frac{\partial\left(\beta m F_{m}(\alpha)\right)}{\partial(1 / \alpha)}
$$

After computing the free-energy $F_{m}(\alpha)$, a Legendre transformation,

$$
\beta m G_{m}(A)=\operatorname{ext}_{\alpha}\left\{\beta m F_{m}(\alpha)-\frac{m(m-1)}{2} N d \frac{A}{\alpha}\right\}
$$

yields a free-energy function $G(A)$. Here extr. $\alpha$ stands for extrimization with respect to $\alpha$. Subsequent extremization of the latter with respect to the cage size $A$,

$$
0=\left.\frac{\partial G_{m}(A)}{\partial A}\right|_{A=A_{\text {extr }}}
$$

yields the cage size $A=A_{\text {extr }}$ of the desired cloned liquid state, which remains after switching-off the artificial confining potential $1 / \alpha \rightarrow 0$. Then the complexity can be computed using Eq. (65). The final step is to find $m^{*}(T)$ at which the complexity vanishes (See Eq. (67)). For clarity we recall the basic steps of the computation [18, 19] in 
the following. Especially the parameter $\alpha=\alpha(A)$ determined below as Eq. (106) is indispensable in our analysis of the rigidity of glasses in sec. III.

Anticipating that thermal fluctuations of the molecular liquid can be factorized into two parts: thermal fluctuations inside molecules and the thermal fluctuations of the CM positions of the molecules, we decompose the positions of the particles as,

$$
\mathbf{r}_{i}^{a}=\mathbf{R}_{i}+\mathbf{u}_{i}^{a}
$$

where $\mathbf{u}_{i}^{a}$ are the fluctuations around the CM position of the molecules at $\mathbf{R}_{i}$. Then the integration variables can be changes as $\int \prod_{i=1}^{N} \prod_{a=1}^{m} d^{d} r_{i}^{a}=\int \prod_{i=1}^{N} d^{d} R_{i} \prod_{a=1}^{m} d^{d} r_{i}^{a} \delta^{d}\left(R_{i}-\frac{1}{m} \sum_{a=1}^{m} r_{i}^{a}\right)=\int \prod_{i=1}^{N} d^{d} R_{i} \prod_{a=1}^{m} d^{d} u_{i}^{a} m^{d} \delta^{d}\left(\sum_{a=1}^{m} u_{i}^{a}\right)$. The averages over thermal fluctuations inside molecules is given by,

$$
\langle\ldots\rangle_{\text {cage }} \equiv \int \prod_{i=1}^{N} \prod_{a=1}^{m} d^{d} u_{i}^{a} P\left(\mathbf{u}_{\mathbf{i}}^{a}\right) \ldots \quad P\left(\left\{\mathbf{u}^{a}\right\}\right) \equiv\left(\frac{(2 \pi \alpha)^{m-1}}{m}\right)^{-d / 2} \delta^{d}\left(\sum_{a=1}^{m} \mathbf{u}^{a}\right) e^{-\frac{1}{4 \alpha} \sum_{a, b=1}^{m}\left|\mathbf{u}^{a}-\mathbf{u}^{b}\right|^{2}}
$$

which yields in particular,

$$
\left\langle\left(u_{i}^{a}\right)^{\mu}\left(u_{j}^{b}\right)^{\nu}\right\rangle_{\text {cage }}=-\left(1-m \delta_{a b}\right) \frac{\alpha}{m^{2}} \delta_{i j} \delta_{\mu \nu}
$$

Assuming first $\alpha$ is small so that the fluctuations $u$ 's are small one finds,

$$
Z_{m}=\frac{1}{N !}{\sqrt{\frac{\left(2 \pi \alpha / \Lambda^{2}\right)^{m-1}}{m^{m}}}}^{N d} m^{d N} \int \prod_{i=1}^{N} \frac{d^{d} R_{i}}{\Lambda^{d}} \prod_{i<j} \exp \left[-\beta m v_{\mathrm{eff}}\left(R_{i}-R_{j}\right)+\ldots\right]
$$

where we introduced the effective potential,

$$
v_{\text {eff }}(r)=v(r)-(1-m) \frac{\alpha}{m^{2}} \nabla^{2} v(r)+\ldots
$$

where $\nabla=(\partial / \partial x, \partial / \partial y, \partial / \partial z)$. This implies averages over thermal fluctuation of the CM positions of the molecules can be written as,

$$
\langle\ldots\rangle_{T / m,\left[v_{\text {eff }}(r)\right]} \equiv \int \prod_{i} d R_{i} W\left(\left\{R_{i}\right\}\right)_{T / m,\left[v_{\text {eff }}(r)\right]} \cdots
$$

with

$$
W\left(\left\{R_{i}\right\}\right)_{T,[v(r)]}=\frac{e^{-\beta \sum_{i<j} v\left(R_{i j}\right)}}{\int \prod_{i=1}^{N} d^{d} R_{i} e^{-\beta \sum_{i<j} v\left(R_{i j}\right)}} .
$$

Now the free-energy of the molecular liquid can be expressed as,

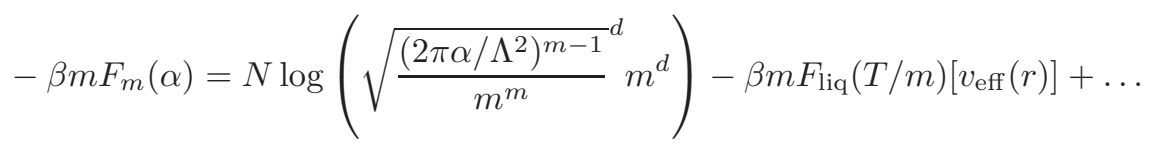

Here the 1 st term on the r.h.s is due to thermal fluctuations inside the molecules. The 2 nd term $F_{\text {liq }}(T / m)[v(r)]$ is the free-energy associated with the configurations of the CM positions of the molecules which turns out to be just the free-energy of a liquid at temperature $T^{*}=T / m$,

$$
-\beta m F_{\text {liq }}(T / m)[v(r)] \equiv \log Z_{\text {liq }}(T / m)[v(r)] \quad Z_{\text {liq }}(T)[v(r)] \equiv \frac{1}{N !} \int \prod_{i=1}^{N} \frac{d^{d} R_{i}}{\Lambda^{d}} e^{-\beta \sum_{i<j} v\left(R_{i}-R_{j}\right)}
$$

Assuming $\alpha$ is small we find,

$$
-\beta m F_{\text {liq }}(T / m)\left[v_{\text {eff }}(r)\right]=-\beta m F_{\text {liq }}(T / m)[v(r)]+\beta(1-m) \frac{\alpha}{m^{2}} \sum_{i<j}\left\langle\nabla^{2} v\left(R_{i j}\right)\right\rangle_{*}+\ldots
$$

where we introduced a short hand notation,

$$
\langle\ldots\rangle_{*} \equiv\langle\ldots\rangle_{T / m,[v(r)]}
$$


The resultant free-energy of the molecular state after the Legendre transformation Eq. (88) is obtained up to 1st order in the expansion of the cage size $A$ as,

$$
\frac{\beta}{N} G_{m}(A)=-\frac{d}{2 m} \log (m)+\frac{d(1-m)}{2 m}+\frac{d(1-m)}{2 m} \log \left(2 \pi A / \Lambda^{2}\right)-A \frac{1-m}{m} \frac{1}{N} \sum_{i<j} \beta\left\langle\nabla^{2} v\left(r_{i j}\right)\right\rangle_{*}+\frac{\beta}{N} F_{\text {liq }}(T / m)+O\left(A^{2}\right)
$$

More precisely the Legendre transformation is done as follows: we expand the parameter $\alpha$ in power series of $A$ as [18],

$$
\alpha=\alpha_{1} A+\alpha_{2} A^{2}+\alpha_{3} A^{3} \ldots
$$

The coefficients $\alpha_{1}$ and $\alpha_{2}$ are fixed by the 1st order cage expansion while $\alpha_{3}$ requires the 2nd order cage expansion.

Since we consider $m \leq 1$, we have to maximize (rather than minimize) the free-energy $G_{m}(A)$ with respect to $A$. The equation $0=\partial G_{m}(A) / \partial A$ Eq. (89) reads,

$$
0=\frac{1}{A}-\frac{2 \beta}{d} \frac{1}{N} \sum_{i<j}\left\langle\nabla^{2} v\left(r_{i j}\right)\right\rangle_{*}+O(A)
$$

Then we find the cage size of the metastable glassy state at the level of the 1st order cage expansion as,

$$
A=\frac{d}{2 \beta \frac{1}{N} \sum_{i<j}\left\langle\nabla^{2} v\left(r_{i j}\right)\right\rangle_{*}} .
$$

Finally the glass transition line $m=m^{*}(T)$ (See Fig. 5) is determined following the prescription discussed in sec. IIC 3. To this end we use $F_{m}(\alpha(A))$ obtained above in Eq. (65) and locate $m=m^{*}(T)$ where the complexity $\Sigma$ vanishes. The Kauzmann transition temperature $T_{\mathrm{K}}$ is determined by Eq. (69).

\section{The effective Einstein model: thermal fluctuations of molecular liquid}

The analysis by the cage expansion implies the molecular liquid state can be described as follows. The coordinates of the particles are decomposed as Eq. (90) which reads,

$$
\mathbf{r}_{i}^{a}=\mathbf{R}_{i}+\mathbf{u}_{i}^{a}
$$

where $\mathbf{R}_{i}$ is the CM position of the molecule and $\mathbf{u}_{i}$ is the deviation of the particle belonging to the $a$-th replica from the CM. The statistical weights of the configurations of the CM are given by Eq. (95) which is that of a liquid with renormalized potential $v_{\text {eff }}(r)$ given by Eq. (94). On the other hand, the statistical weights of the configuration of the displacements inside the molecules are given by Eq. (91)). Therefore the thermal average in the molecular liquid state becomes factorized as,

$$
\langle\ldots\rangle=\left\langle\langle\ldots\rangle_{\text {cage }}\right\rangle_{T / m,\left[v_{\text {eff }}(r)\right]}=\int \prod_{i=1}^{N} d^{d} R_{i} W_{T / m,\left[v_{\text {eff }}(r)\right]}\left(\left\{R_{i}\right\}\right) \prod_{a=1}^{m} d^{d} u_{i}^{a} P\left(\left\{u_{i}^{a}\right\}\right)
$$

where $\langle\ldots\rangle_{\text {cage }}$ is the average over thermal fluctuations inside the molecules defined in Eq. (91)) and $\langle\ldots\rangle_{T / m,\left[v_{\text {eff }}(r)\right]}$ is the average over thermal fluctuations of the CM positions of the molecules defined in Eq. (95).

The key parameter which defines the renormalized potential $v_{\text {eff }}(r)$ and the width of the fluctuations inside molecules is the parameter $\alpha$. Going back to Eq. (102) we find the parameter $\alpha=\alpha(A)$ as,

$$
\frac{\alpha}{m^{2}}=2 \frac{A}{m}+O\left(A^{3}\right)=\frac{d}{\beta^{*} \frac{1}{N} \sum_{i<j}\left\langle\nabla^{2} v\left(r_{i j}\right)\right\rangle_{*}}+O\left(A^{3}\right)
$$

where the cage size $A$ is given by Eq. (104). This follows from the fact that $\alpha_{1} A=m A$ and $\alpha_{2} A^{2}=m A$ (the latter can be seen using Eq.(38) and (40) of [18]). The result implies in particular that,

$$
\left\langle\left(u_{i}^{a}\right)^{\mu}\left(u_{j}^{b}\right)^{\nu}\right\rangle_{\text {cage }}=\left\langle u^{2}\right\rangle_{\text {cage }} \delta_{i j} \delta_{\nu \mu}\left(m \delta_{a b}-1\right) \quad\left\langle u^{2}\right\rangle_{\text {cage }} \equiv \frac{d}{\frac{\beta^{*}}{N} \sum_{i<j}\left\langle\nabla^{2} v\left(r_{i j}\right)\right\rangle_{*}}=\frac{k_{\mathrm{B}} T^{*}}{\kappa_{\mathrm{eff}}}
$$


where we introduced

$$
\kappa_{\mathrm{eff}} \equiv \frac{\frac{1}{N} \sum_{i<j}\left\langle\nabla^{2} v\left(r_{i j}\right)\right\rangle_{*}}{d}
$$

Eq. (107) follows from Eq. (91), Eq. (92) and Eq. (106).

It is instructive to note that the above result could have been obtained without using replicas but more intuitively. Suppose that we focus on a single particle, say $i$-th particle, and imagine that all other particles $j(\neq i)$ are $f r o z e n$ in a certain configuration. Then the surrounding particles create a static effective potential $U_{i}=\frac{1}{2} \sum_{\mathrm{J}}(\neq i) v\left(r_{i j}\right)$ for the $i$-th particle: the Einstein model. Then the thermal average of the fluctuation of the particle can be evaluated as,

$$
\left\langle\mathbf{u}_{i}^{2}\right\rangle \simeq \frac{k_{\mathrm{B}} T}{(1 / 2) \sum_{j(\neq i)} \nabla^{2} v\left(r_{i j}\right) / d}
$$

Here we assumed isotropic fluctuations inside cages. Thus we find $\kappa_{\text {eff }}$ defined in Eq. (108) can be viewed as the Hookian spring constant of the effective Einstein oscillator.

The above discussion suggests that the basic idea of cage expansion is very similar in spirit to consider energy landscape in the vicinity of inherent structures (see sec. ПA5). However there is an important difference : the configurations of the center of mass positions of the molecules $R_{i}$ are by no means restricted to energy minima but allowed to take any configurations of a liquid thermalized at effective temperature $T_{\text {eff }}=T / m^{*}$ (see Eq. (95)). As we discuss soon, this point is important in linear responses to external perturbations at finite temperatures.

Using the statistical weights Eq. 105 thermal averages of physical quantities can be done as follow. Consider an observable $O\left(\left\{r_{i j}^{a}\right\}\right)$ which is a function of the distances between particles $r_{i j}^{a}=\left|\mathbf{r}_{i}^{a}-\mathbf{r}_{j}^{a}\right|$. Using the molecular coordinate Eq. (90) and assuming that the fluctuations $u_{i}^{a}$ 's are small, it can be expanded as,

$$
\begin{aligned}
O\left(\left\{r_{i j}^{a}\right\}\right)= & O\left(\left\{R_{i j}\right\}\right)+\sum_{i<j} \sum_{a} \sum_{\mu} \frac{\partial O\left(\left\{r_{i j}^{a}\right\}\right)}{\partial\left(r_{i j}^{a}\right)^{\mu}}\left(u_{i}^{a}-u_{j}^{a}\right)^{\mu} \\
& +\frac{1}{2} \sum_{i<j, k<l} \sum_{a, b} \sum_{\mu, \nu} \frac{\partial^{2} O\left(\left\{r_{i j}^{a}\right\}\right)}{\partial\left(r_{i j}^{a}\right)^{\mu} \partial\left(r_{k l}^{b}\right)^{\nu}}\left(u_{i}^{a}-u_{j}^{a}\right)^{\mu}\left(u_{k}^{b}-u_{l}^{b}\right)^{\nu} \ldots
\end{aligned}
$$

Then the thermal average can be done as,

$$
\begin{aligned}
\left\langle O\left(\left\{r_{i j}^{a}\right\}\right)\right\rangle & =\left\langle O\left(\left\{R_{i j}\right\}\right)\right\rangle_{T / m,\left[v_{\text {eff }}(r)\right]}-\frac{1}{2}\left\langle u^{2}\right\rangle_{\text {cage }} \sum_{i} \sum_{j_{1}(\neq i)} \sum_{j_{2}(\neq i)} \sum_{a, b}\left(1-m \delta_{a b}\right) \sum_{\mu}\left\langle\frac{\partial^{2} O\left(\left\{r_{i j}^{a}\right\}\right)}{\partial\left(r_{i j_{1}}^{a}\right)^{\mu} \partial\left(r_{i j_{2}}^{b}\right)^{\mu}}\right\rangle_{*}+\ldots \\
& =\left\langle O\left(\left\{R_{i j}\right\}\right)\right\rangle_{*}+\beta^{*}(1-m)\left\langle u^{2}\right\rangle_{\text {cage }} \sum_{i<j}\left[\left\langle O\left(\left\{R_{i j}\right\}\right) \nabla^{2} v\left(R_{i j}\right)\right\rangle_{*}-\left\langle O\left(\left\{R_{i j}\right\}\right)\right\rangle_{*}\left\langle\nabla^{2} v\left(R_{i j}\right)\right\rangle_{*}\right]+\ldots \\
& -\frac{1}{2}\left\langle u^{2}\right\rangle_{\text {cage }} \sum_{i} \sum_{j_{1}(\neq i)} \sum_{j_{2}(\neq i)} \sum_{a, b}\left(1-m \delta_{a b}\right) \sum_{\mu}\left\langle\frac{\partial^{2} O\left(\left\{r_{i j}^{a}\right\}\right)}{\partial\left(r_{i j_{1}}^{a}\right)^{\mu} \partial\left(r_{i j_{2}}^{b}\right)^{\mu}}\right\rangle_{*}+\ldots
\end{aligned}
$$

where $\alpha / m^{2}=2 A / m$ as given in Eq. (106). The evaluation of the averages $\langle\ldots\rangle_{*}$ (see Eq. (100)) can be done using methods of standard liquid theory [23] at temperature $T / m$. The value of the cage size $A=A(T)$ and the parameter $m=m^{*}(T)$ must be determined beforehand.

\section{Evaluations of intra-state and inter-state thermal fluctuations by the cage expansion}

In sec. $I \mathrm{C} 4$ we discussed the possibility to disentangle the intra-state and inter-state responses using a cloned system. Here we present a more precise prescription to do it explicitly using the cage expansion. In Appendix B we demonstrate that the prescription works exactly in a mean-field spin-glass model. In the next section we compute the rigidity applying the following prescription to evaluate shear-stress correlation function which appears in the static fluctuation formula of the rigidity Eq. (8).

Here let us consider the generic susceptibility considered in sec. II C4 (see Eq. (80)),

$$
\chi_{a b}=\frac{\beta}{N}\left(\left\langle O_{a} O_{b}\right\rangle-\left\langle O_{a}\right\rangle\left\langle O_{b}\right\rangle\right) .
$$


Using the above prescription Eq. (111) it becomes,

$$
\begin{aligned}
\chi_{a b}= & \frac{\beta}{N}\left(\left\langle O^{2}\left(\left\{R_{i j}\right\}\right)\right\rangle_{T / m,\left[v_{\text {eff }}(r)\right]}-\left\langle O\left(\left\{R_{i j}\right\}\right)\right\rangle_{T / m,\left[v_{\text {eff }}(r)\right]}^{2}\right) \\
& -\frac{\beta}{N} \sum_{i j} \sum_{\mu \nu}\left\langle\frac{\partial O}{\partial\left(x_{i}^{a}\right)^{\mu}} \frac{\partial O}{\partial\left(x_{j}^{b}\right)^{\nu}}\right\rangle_{*}\left(1-m \delta_{a b}\right)\left\langle\left(u_{i}\right)^{\mu}\left(u_{j}\right)^{\nu}\right\rangle_{\text {cage }}
\end{aligned}
$$

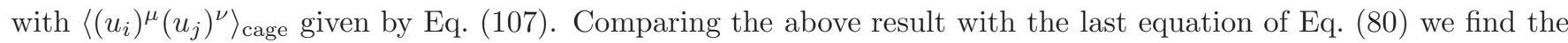
intra-state susceptibility as,

$$
\hat{\chi}=\frac{\beta^{*}}{N} \sum_{i j} \sum_{\mu \nu}\left\langle\frac{\partial O}{\partial\left(x_{i}^{a}\right)^{\mu}} \frac{\partial O}{\partial\left(x_{j}^{b}\right)^{\nu}}\right\rangle_{*}\left\langle\left(u_{i}\right)^{\mu}\left(u_{j}\right)^{\nu}\right\rangle_{\text {cage }}=\frac{\beta^{*}}{N} \sum_{i} \sum_{\mu}\left\langle\left(\frac{\partial O}{\partial\left(x_{i}^{a}\right)^{\mu}}\right)^{2}\right\rangle_{*}\left\langle u^{2}\right\rangle_{\text {cage }}
$$

with $\beta^{*}=m \beta$, and the inter-state susceptibility,

$$
\tilde{\chi}=\frac{\beta}{N}\left(\left\langle O^{2}\left(\left\{R_{i j}\right\}\right)\right\rangle_{T / m,\left[v_{\text {eff }}(r)\right]}-\left\langle O\left(\left\{R_{i j}\right\}\right)\right\rangle_{T / m,\left[v_{\text {eff }}(r)\right]}^{2}\right)-\frac{\beta}{N} \sum_{i} \sum_{\mu}\left\langle\left(\frac{\partial O}{\partial\left(x_{i}^{a}\right)^{\mu}}\right)^{2}\right\rangle_{*}\left\langle u^{2}\right\rangle_{\text {cage }}
$$

Finally the total susceptibility Eq. (84) becomes,

$$
\chi=\frac{\beta^{*}}{N}\left(\left\langle O^{2}\left(\left\{R_{i j}\right\}\right)\right\rangle_{T / m,\left[v_{\mathrm{eff}}(r)\right]}-\left\langle O\left(\left\{R_{i j}\right\}\right)\right\rangle_{T / m,\left[v_{\mathrm{eff}}(r)\right]}^{2}\right)
$$

Although we have only performed the cage expansion up to 1 st order in Eq. (114),Eq. (115) and Eq. (116), we expect that the basic structure of the result is generic. Higher order cage expansion will yield more accurate effective potential $v_{\text {eff }}(r)$ [20] which will refine the averages over the CM positions of the molecules.

\section{Discussion: responses inside metastable states}

Let us focus on the responses within metastable states and discuss it more in detail. Since fluctuations in the close vicinity of an IS is completely characterized by the elastic hamiltonian Eq. (34), the susceptibility $\chi_{\text {IS }}$ associated with an IS can be obtained just like the 1st equation of Eq. (114) but replacing the correlator $\left\langle\left(u_{i}\right)^{\mu}\left(u_{j}\right)^{\nu}\right\rangle_{\text {cage }}$ by,

$$
\left\langle\left(u_{i}\right)^{\mu}\left(u_{j}\right)^{\nu}\right\rangle_{\mathrm{IS}}=\left(\beta H^{-1}\right)_{i j}^{\mu \nu}
$$

with $H^{-1}$ being the inverse of the Hessian matrix Eq. (36). As the result we find,

$$
\chi_{\mathrm{IS}}=\left.\left.\frac{1}{N} \sum_{i j} \sum_{\mu \nu} \frac{\partial O}{\partial\left(x_{i}^{a}\right)^{\mu}}\right|_{\mathrm{IS}} \frac{\partial O}{\partial\left(x_{j}^{b}\right)^{\nu}}\right|_{\mathrm{IS}}\left(H^{-1}\right)_{i j}^{\mu \nu}
$$

where the derivatives are took at the IS. This must be compared with the intra-state susceptibility $\hat{\chi}$ defined in Eq. (114) together with Eq. (107), which reads,

$$
\hat{\chi}=\frac{1}{N} \sum_{i} \sum_{\mu}\left\langle\left(\frac{\partial O}{\partial\left(x_{i}^{a}\right)^{\mu}}\right)^{2}\right\rangle \frac{1}{\kappa_{\mathrm{eff}}}
$$

where we used Eq. (108).

Comparing the two susceptibilities $\chi_{\text {IS }}$ and $\hat{\chi}$ we find that they are apparently similar to some extent. In both cases, the fluctuation of the observable $O$ is induced by small fluctuation of particles around some reference state(s). We also notice that the cage expansion approach amounts to replace the full Hessian matrix by that of an effective Einstein model we discussed in sec. II C6. Validity of such an approximation should be examined critically. A better approximation may be obtained for example by doing partial harmonic re-summation exactly [18].

However here let us focus on a more important difference between the two susceptibilities that comes from the difference of their reference state $(s)$ themselves. Note that the reference state for $\hat{\chi}$ as defined in Eq. (119) is a thermalized liquid configuration within a metastable state at a finite temperature $T^{*}=T / m^{*}(T)$, where all sorts of 
thermal excitations, not only the elastic but also plastic deformations, are included. On the other hand the reference state for $\chi_{\mathrm{IS}}$ is just an energy minimum. This implies for instance,

$$
\left(\left.\frac{\partial O}{\partial\left(x_{i}^{a}\right)^{\mu}}\right|_{\mathrm{IS}}\right)^{2}<\left\langle\left(\frac{\partial O}{\partial\left(x_{i}^{a}\right)^{\mu}}\right)^{2}\right\rangle .
$$

The above observation suggests that in general the intra-state susceptibility $\hat{\chi}$ is larger than that of the inherent structures $\chi_{\mathrm{IS}}$,

$$
\hat{\chi}>\chi_{\mathrm{IS}} .
$$

In sec ПA5 we argued that a metastable would be identified as a metabasin (MB), which is a union of inherent structures (IS), rather than a single IS. Then the difference between the two susceptibilities Eq. (121) should be attributed to thermal fluctuations between different inherent structures within a common metabasin, which are necessarily plastic deformations. In the supercooled liquid state $T>T_{K}, m^{*}(T)=1$ so that $T^{*}=T$. This implies the difference between the two susceptibilities increases with temperature at $T>T_{K}$. On the other hand, in the glass phase $T<T_{K}$ one typically finds $m^{*}(T) \sim T / T_{\mathrm{K}}$ so that $T^{*}=T / m^{*}(T) \sim T_{\mathrm{K}}[18,19]$. This suggests that $\hat{\chi}$ becomes nearly independent of temperatures below $T_{\mathrm{K}}$ such that $\tilde{\chi} \sim \chi_{\mathrm{IS}}$.

We argued in sec. 【A 4 that the same intra-state susceptibility can be observed either in equilibrium or out-of equilibrium based on the observation that in general the $\beta$-relaxation shows little aging effects. In this respect, it is interesting to note that the intra-state susceptibility $\hat{\chi}$ reflect the underlying equilibrium state. Especially the above discussion suggests even the Kauzmann temperature $T_{\mathrm{K}}$ itself may be reflected in the temperature dependence of $\hat{\chi}$.

\section{RIGIDITY OF GLASSES}

\section{A. Shear on the cloned liquid}

In sec. 【A1 and sec. 【A2 we discussed some general aspects of static response to shear. The rigidity is defined as the 2nd derivative of the free-energy with respect to the shear-strain $\gamma$ as in Eq. (2) whose explicit expression, the fluctuation formula, is given in Eq. (8). Now let us consider to shear the cloned liquid. Suppose that we have a cloned liquid of $m$ replicas which are subjected different shear-strains $\gamma^{a}(a=1,2, \ldots, m)$ : the replicas are put into different containers which can be deformed independently. This is just an example of the static perturbations on the cloned liquid discussed in sec. IIC4. Then we may formally expand the free-energy of the cloned liquid as,

$$
m F_{m}(\gamma)=m F_{m}(0)+N \sum_{a=1}^{m}\left\langle\sigma^{a}\right\rangle \gamma_{a}+\frac{N}{2} \sum_{a, b=1}^{m} \mu_{a b} \gamma^{a} \gamma^{b}+O\left(\gamma^{d}\right)
$$

The argument for the decomposition of the response Eq. (80) implies that the generalized rigidity $\mu_{a b}$ may also be parametrized as,

$$
\mu_{a b}=\hat{\mu} \delta_{a, b}+\tilde{\mu}
$$

with the intra-state rigidity or effective rigidity,

$$
\hat{\mu}=\left[\left[\left.\frac{\partial^{2} f_{\alpha}(T, \gamma)}{\partial \gamma^{2}}\right|_{\gamma=0}\right]\right]_{T, m}
$$

and the negative correction term due to inter-state fluctuations,

$$
\tilde{\mu}=-N \beta\left(\llbracket \sigma_{\alpha}^{2} \rrbracket_{T, m}-\llbracket \sigma_{\alpha} \rrbracket_{T, m}^{2}\right)
$$

where $\sigma_{\alpha}$ is the 'internal stress' of state $\alpha$,

$$
\sigma_{\alpha}=\left.\frac{\partial f_{\alpha}(T, \gamma)}{\partial \gamma}\right|_{\gamma=0}
$$

As we discuss below $\tilde{\mu}$, which is negative, can be interpreted as inter-state modulus. 


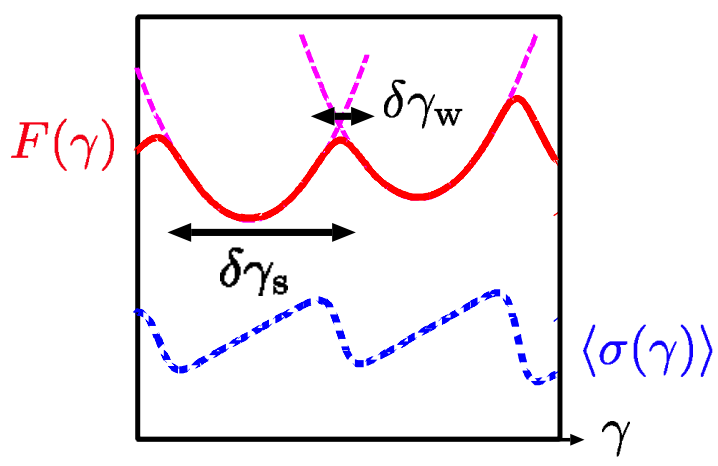

FIG. 6. Schematic mean-field picture of free-energy landscape and static stress-strain curve below $T_{\mathrm{K}}$. The metastable states (metabasins) with rigidity $\hat{\mu}$ are represented by parabolas

The trace over the replica index of $\mu_{a b}$ yields the rigidity of a molecular liquid. This must be zero since cloned liquid as a whole is just a liquid (!) (see the discussion in the end of sec. П1 2)

$$
\sum_{b=1}^{m} \mu_{a b}=\hat{\mu}+m \tilde{\mu}=0
$$

Following the discussion in sec. 【IC4 we find the total rigidity of the glass from Eq. (127) as,

$$
\mu=\lim _{m \rightarrow 1^{-}} \sum_{b=1}^{m} \mu_{a b}=\hat{\mu}+m^{*} \tilde{\mu}=0 .
$$

where $m^{*}=m^{*}(T)$ is the glass transition line. Thus we find the inter-state correction term $\hat{\mu}$ is simply related to the intra-state rigidity $\hat{\mu}$ as,

$$
\tilde{\mu}=-\frac{1}{m^{*}} \hat{\mu}
$$

\section{B. Static analogue of yielding or shocks}

Let us discuss a physical interpretation of the above result Eq. (129). Suppose that internal stress $\sigma_{\alpha}$ defined in Eq. (126) has a Gaussian distribution with zero mean and variance $\Delta^{2} / N$. Then Eq. (125) and Eq. (129) implies that the intra-state rigidity is related to $\Delta$ as [49],

$$
\hat{\mu}=m^{*} \beta \Delta^{2} .
$$

Note that this corresponds to Eq. (32). (We mentioned similar relations for the instantaneous rigidity Eq. (17) and the rigidity of inherent structures Eq. (31)). A very recent numerical study [112] explicitly demonstrated that the internal stresses of inherent structures indeed follow Gaussian distributions.

The static response of a system with many metastable states against variation of the shear-strain $\gamma$ at low temperatures $T<T_{\mathrm{K}}$ may be viewed pictorially as shown in Fig. [6] Variation of the free-energy $f_{\alpha}(\gamma)$ associated with a given metastable state $\alpha$ along the $\gamma$-axis may be described as,

$$
f_{\alpha}(\gamma)=\frac{\hat{\mu}}{2}\left(\gamma-\gamma_{\alpha}^{\min }\right)^{2}+f_{\alpha}^{\min }
$$

where $\hat{\mu}$ is the intra-state rigidity. The free-energy takes a minimum value $f_{\alpha}^{\min }$ at $\gamma=\gamma_{\alpha}^{\min }$. Then the internal stress $\sigma_{\alpha}(\gamma)$ of the metastable state at a given $\gamma$ is given by,

$$
\sigma_{\alpha}(\gamma)=\hat{\mu}\left(\gamma-\gamma_{\alpha}^{\min }\right)
$$

Of course we should expect that the metastable state itself disappears at some distance from $\gamma_{\alpha}^{\min }$ approaching its metastability limit where a saddle-node bifurcation takes place [63]. 
Let us consider a set of metastable states which lives at around a given point $\gamma$ along the $\gamma$-axis. The locations of their minima $\gamma_{\alpha}^{\min }$ will be distributed randomly around the point $\gamma$. Then the internal stress $\sigma_{\alpha}(\gamma)$ defined by Eq. (132) at a given $\gamma$ will take both positive and negative random values depending on the sign of $\gamma-\gamma_{\alpha}^{\min }$. Then the relation Eq. (130) implies the variance of the distribution of the distances $\gamma-\gamma_{\alpha}^{\min }$ is $\left(1 / m^{*} \beta \hat{\mu}\right) N^{-1}$.

At any point along the $\gamma$-axis, there will be exponentially large number of metastable states $\propto e^{N \Sigma(f)}$ at a given free-energy level (per particle) $f$ where $\Sigma(f)$ is the complexity or structural entropy Eq. (63). However, at low temperatures $T<T_{\mathrm{K}}$, the ground state and at most only a few other low lying states would have significant statistical weights [92]. Under variation of $\gamma$, there will be level crossings among such low lying states as shown in Fig. 6. Just around the points where there are level crossings, the rigidity should become locally negative. Correspondingly the ground state energy exhibits a cusp singularity where a level crossing takes place. This would be the physical interpretation of the negative inter-state modulus $\tilde{\mu}=-\left(1 / m^{*}\right) \hat{\mu}$ (See Eq. (129)). We can consider this feature as a static analogue of yielding.

Typical spacing between such level crossings along the $\gamma$-axis will be of order,

$$
\delta \gamma_{\mathrm{s}}=\frac{k_{\mathrm{B}} T_{\mathrm{K}}}{\Delta \sqrt{N}} .
$$

This is because typical free-energy gap between low lying metastable states will be of order $k_{\mathrm{B}} T_{\mathrm{K}}$ while free-energy change of each of them for a small change of the strain $\gamma$ is proportional to their internal stress $\sigma_{\alpha}$. Another important scale is typical width of the thermal rounding of the level crossings. This would be simply give by,

$$
\delta \gamma_{\mathrm{w}}=\frac{k_{\mathrm{B}} T}{\Delta \sqrt{N}} .
$$

In the zero temperature limit $T \rightarrow 0$, the free-energy landscape should exhibit cusp singularity. Correspondingly we find that the intra-state modulus $\tilde{\mu}$ defined in Eq. (129) diverges negatively because $\lim _{T \rightarrow 0} m^{*}(T) \rightarrow 0$. [93],

Based on the above observations the probability $p_{\text {crossing }}$ to meet a level crossing at a given $\gamma$ may be estimated as

$$
p_{\text {crossing }} \sim \delta \gamma_{\mathrm{w}} / \delta \gamma_{\mathrm{s}} \sim T / T_{\mathrm{K}}
$$

It is interesting to recall that the factor $m^{*}(T)$ which appears in front of the inter-state modulus $\hat{\mu}$ in Eq. (128) can be regarded as the probability to make an inter-state transitions within the standard interpretation of the replica symmetry breaking ansatz 90, 91]. (See sec. [IC4 and Appendix B for related discussions) Typically it is found that $m^{*}(T) \sim T / T_{\mathrm{K}}[18,19]$. Thus Eq. (135) is consistent with the standard interpretation of the RSB ansatz.

Note that in the thermodynamic limit $N \rightarrow \infty$, the spacing between the cusps vanishes [93, 94]. It means that if we define a coarse-grained rigidity for any small but non-zero interval along the $\gamma$-axis, we obtain 0 rigidity in the $N \rightarrow \infty$ limit. Thus the static intermittency of the stress-strain curve sketched in Fig. 6 explains how we can solve the apparent paradox discussed in sec. [I 1 in the static mean-field theoretical context. Similar static intermittencies have been found in disordered systems including spin-glasses [93 98], and elastic manifolds in random media [99 101], which are actually related to the problem of intermittency in turbulent flows due to shocks [102].

The above observation implies the inter-state fluctuations can be considered as Goldstone modes characteristic in glasses which try to restore the broken translational symmetry. On the other hand, the elastic fluctuation within a metastable state is more usual Goldstone mode which is also present in crystals.

Although we have assumed $T<T_{\mathrm{K}}$ in the above discussions, the transitions between different metastable states under external strain can be relevant even at higher temperatures in the following sense. Within the so called mosaic picture of the RFOT theory [7, 9, 16, 17], the configuration of the system in the intermediate temperature range $T_{\mathrm{K}}<T<T_{\mathrm{c}}$ consists of mosaics or patches of local regions such that the subsystem in each of the patch stays in a metastable state. The size of the local regions is called as the mosaic length $\xi_{\text {mosaic }}$. It means that the subsystem within a mosaic is as if at a temperature below $T_{\mathrm{K}}$.

Then an interesting possibility is that the intermittent stress-strain curves shown schematically in Fig. 6 may be observable by some rheological experiment in a confined geometry. Suppose to exert some local shear-strain $\gamma$ in a quasi-static manner on a system confined in a narrow region of size $L$. If the size of the region is small enough such that $L<\xi_{\text {mosaic }}$, then the resultant stress-strain curve may exhibit the intermittent feature with typical spacing between the shocks $\delta \gamma_{\mathrm{s}} \propto 1 / \sqrt{L^{d}}$.

Finally also note for clarity that yielding at mesoscopic scales can occur by the saddle-node bifurcation mechanism at metastability limits 32 , 33, 77]. If the energy level at the metastability limit is order $O(1)$, we find again that $\delta \gamma_{\mathrm{s}} \propto 1 / \sqrt{L^{d}}$. 


\section{Cage expansion of the rigidity}

Now let us examine the rigidity $\mu_{a b}$ defined in Eq. (122). Its microscopic expression can be obtained generalizing the fomulae Eqs. Eq. (8), Eq. (9) and Eq. (10),

$$
\mu_{a b}=\frac{\partial^{2} m F_{m}}{\partial \gamma^{a} \partial \gamma^{b}}=\left\langle b_{\left[\left\{\mathbf{r}_{i}^{a}\right\}\right]}\right\rangle \delta_{a b}-\beta\left[\left\langle\sigma_{\left[\left\{\mathbf{r}_{i}^{a}\right\}\right]} \sigma_{\left[\left\{\mathbf{r}_{i}^{b}\right\}\right]}\right\rangle-\left\langle\sigma_{\left[\left\{\mathbf{r}_{i}^{a}\right\}\right]}\right\rangle\left\langle\sigma_{\left[\left\{\mathbf{r}_{i}^{b}\right\}\right]}\right\rangle\right] .
$$

where the subscript, for instance, $\left[\left\{\mathbf{r}_{i}^{a}\right\}\right]$ indicates that coordinates $\mathbf{r}_{i}(i=1,2, \ldots, N)$ of particles in replica $a$. The 1 st term $b$ on the r.h.s of the above equation is the born term defined in Eq. (9) which reads,

$$
b_{\left[\left\{\mathbf{r}_{i}\right\}\right]}=\frac{1}{N} \sum_{i<j} b\left(\mathbf{r}_{i j}\right) \quad b\left(\mathbf{r}_{i j}\right)=\hat{z}_{i j}^{2}\left[r^{2} v^{(2)}(r) \hat{x}_{i j}^{2}+r v^{(1)}(r)\left(1-\hat{x}_{i j}^{2}\right)\right]_{r=r_{i j}} .
$$

The 2nd term is the correlation function of the fluctuation of the shear-stress,

$$
\sigma_{\left[\left\{\mathbf{r}_{i}\right\}\right]}=\frac{1}{N} \sum_{i<j} \sigma\left(\mathbf{r}_{i j}\right) \quad \sigma\left(\mathbf{r}_{i j}\right)=\left.r v^{(1)}(r)\right|_{r=r_{i j}} \hat{x}_{i j} \hat{z}_{i j} .
$$

Our task is to evaluate the rigidity $\mu_{a b}$ by the cage expansion as follows. It is essentially the same as the analysis of the generic susceptibility $\chi_{a b}$ we discussed in sec. IIC7 First we explicitly decompose the coordinates of the particles as Eq. (90),

$$
\mathbf{r}_{i}^{a}=\mathbf{R}_{i}+\mathbf{u}_{i}^{a} .
$$

Then we expand the thermal averages which appear in the fluctuation formula of the rigidity Eq. (136) in power series of the molecular coordinates $\mathbf{u}_{i}^{a}(a=1,2, \ldots, m, i=1,2, \ldots, N)$ using the formula Eq. (111). Then we plug-in the expansions in the microscopic expression of the the rigidity $\mu_{a b}$ given by Eq. (136). Finally we evaluate the thermal averages using Eq. (91) (Eq. (92)) together with Eq. (106) for the molecular coordinates $\mathbf{u}_{i}^{a}$ and Eq. (100) for the center of mass coordinates $\mathbf{R}_{i}$ of the molecules. The result may be put into a power series of the parameter $\alpha$ (or the cage size $A$ ),

$$
\mu_{a b}=\left(\mu_{0}\right)_{a b}+\left(\mu_{1}\right)_{a b} \alpha+O\left(\alpha^{2}\right)
$$

or

$$
\mu_{a b}=\hat{\mu} \delta_{a b}+\tilde{\mu} \quad \hat{\mu}=\hat{\mu}_{0}+\hat{\mu}_{1} \alpha+O\left(\alpha^{2}\right) \quad \tilde{\mu}=\tilde{\mu}_{0}+\tilde{\mu}_{1} \alpha+O\left(\alpha^{2}\right)
$$

which must satisfy the sum rule Eq. (127),

$$
\sum_{b}\left(\mu_{n}\right)_{a b}=\hat{\mu}_{n}+m \tilde{\mu}_{n}=0
$$

at each other in the cage expansion.

\section{Zero-th order}

At zero-th order of the cage expansion, all particles are bound to the CM positions of the molecules. We find,

$$
\left(\mu_{0}\right)_{a b}=\left\langle b_{\left[\left\{\mathbf{R}_{i}\right\}\right]}\right\rangle_{*} \delta_{a b}-\beta\left[\left\langle\sigma_{\left[\left\{\mathbf{R}_{i}\right\}\right]}^{2}\right\rangle_{*}-\left\langle\sigma_{\left[\left\{\mathbf{R}_{i}\right\}\right]}\right\rangle_{*}^{2}\right]
$$

which obviously satisfies the sum rule Eq. (141),

$$
\sum_{a=1}^{m}\left(\mu_{0}\right)_{a b}=\left\langle b_{\left[\left\{\mathbf{R}_{i}\right\}\right]}\right\rangle_{*}-\beta m\left[\left\langle\sigma_{\left[\left\{\mathbf{R}_{i}\right\}\right]}^{2}\right\rangle_{*}-\left\langle\sigma_{\left[\left\{\mathbf{R}_{i}\right\}\right]}\right\rangle_{*}^{2}\right]=0,
$$

since this is just the rigidity of a simple liquid at $T=T / m^{*}$. Thus at the zero-th order we find the intra-state and inter-state rigidities as,

$$
\hat{\mu}_{0}=\left\langle b_{\left[\left\{\mathbf{R}_{i}\right\}\right]}\right\rangle_{*} \quad \tilde{\mu}_{0}=-\frac{\hat{\mu}_{0}}{m} .
$$




\section{First order}

At the 1st order of the cage expansion we find the following result,

$$
\left(\mu_{1}\right)_{a b} \alpha=J_{1}\left(1-m \delta_{a b}\right)+(1-m)\left[\left(J_{2}+J_{3}\right) \delta_{a b}+J_{4}+J_{5}\right]
$$

where

$$
\begin{aligned}
& J_{1}=c \frac{1}{N} \sum_{i} \sum_{j_{1}(\neq i)} \sum_{j_{2}(\neq i)} \beta\left\langle\nabla \sigma\left(\mathbf{r}_{i j_{1}}\right) \cdot \nabla \sigma\left(\mathbf{r}_{i j_{2}}\right)\right\rangle_{*} \\
& J_{2}=-c \frac{1}{N} \sum_{i<j}\left\langle\nabla^{2} b\left(\mathbf{r}_{i j}\right)\right\rangle_{*} \\
& J_{3}=c \frac{1}{N} \sum_{i<j} \sum_{k<l} \beta^{*}\left[\left\langle b\left(\mathbf{r}_{i j}\right) \nabla^{2} v\left(r_{k l}\right)\right\rangle_{*}-\left\langle b\left(\mathbf{r}_{i j}\right)\right\rangle_{*}\left\langle\nabla^{2} v\left(r_{k l}\right)\right\rangle_{*}\right] \\
& J_{4}=2 c \frac{1}{N} \sum_{i_{1}<j_{1}} \sum_{i_{2}<j_{2}} \beta\left[\left\langle\nabla^{2} \sigma\left(\mathbf{r}_{i_{1} j_{1}}\right) \sigma\left(\mathbf{r}_{i_{2} j_{2}}\right)\right\rangle_{*}-\left\langle\nabla^{2} \sigma\left(\mathbf{r}_{i_{1} j_{1}}\right)\right\rangle_{*}\left\langle\sigma\left(\mathbf{r}_{i_{2} j_{2}}\right)\right\rangle_{*}\right] \\
& J_{5}=-c \frac{1}{N} \sum_{i_{1}<j_{1}} \sum_{i_{2}<j_{2}} \sum_{k<l} \beta \beta^{*}\left[\left\langle\sigma\left(\mathbf{r}_{i_{1} j_{1}}\right) \sigma\left(\mathbf{r}_{i_{2} j_{2}}\right) \nabla^{2} v\left(r_{k l}\right)\right\rangle_{*}-\left\langle\sigma\left(\mathbf{r}_{i_{1} j_{1}}\right) \sigma\left(\mathbf{r}_{i_{2} j_{2}}\right)\right\rangle_{*}\left\langle\nabla^{2} v\left(r_{k l}\right)\right\rangle_{*}\right]
\end{aligned}
$$

Here we introduced a parameter,

$$
c \equiv\left\langle u^{2}\right\rangle_{\mathrm{cage}}=\frac{1}{\beta^{*} \kappa_{\mathrm{eff}}}
$$

with $\kappa_{\text {eff }}$ defined in Eq. (108). In the above equations Eq. (146) and in the following quantities which appear inside the brackets $\langle\ldots\rangle_{*}$ are functions of only the CM coordinates $\mathbf{R}_{i}(i=1,2, \ldots, N)$.

As discussed in Appendix D, the group of terms $J_{2}, J_{3}, J_{4}, J_{5}$ can be related to the rigidity of renormalized liquid interacting with the effective potential $v_{\text {eff }}(r)$ given in Eq. (94). Then the following identity,

$$
J_{2}+J_{3}+m\left(J_{4}+J_{5}\right)=0,
$$

holds. Using this identity, the result Eq. (145) can be simplified as,

$$
\mu_{1} \alpha=\left(\hat{\mu}_{1} \delta_{a b}+\tilde{\mu}\right) \alpha=\left[J_{1}-\frac{1-m}{m}\left(J_{2}+J_{3}\right)\right]\left(1-m \delta_{a b}\right) .
$$

so that

$$
\hat{\mu}_{1} \alpha=-m\left[J_{1}-\frac{1-m}{m}\left(J_{2}+J_{3}\right)\right] \quad \tilde{\mu}_{1} \alpha=J_{1}-\frac{1-m}{m}\left(J_{2}+J_{3}\right)
$$

The result satisfies the sum rule Eq. (141),

$$
\sum_{b}\left(\mu_{1}\right)_{a b}=0
$$

as it should. To summarize, up to 1st order in the cage expansion we find,

$$
\hat{\mu}=\left\langle b_{\left[\left\{\mathbf{R}_{i}\right\}\right]}\right\rangle_{*}-m J_{1}+\left(J_{2}+J_{3}\right)(1-m) \quad \tilde{\mu}=-\frac{\hat{\mu}}{m} .
$$

\section{Summary and discussions}

To summarize, we find the rigidity of the cloned liquid Eq. (136) as,

$$
\begin{aligned}
& \mu_{a b}=\hat{\mu}\left(\delta_{a b}-\frac{1}{m}\right) \\
& \hat{\mu}=\left\langle b^{\mathrm{eff}}\right\rangle_{T / m,\left[v_{\text {eff }}(r)\right]}-c \beta^{*} \frac{1}{N} \sum_{i}\left\langle\left|\boldsymbol{\Xi}_{i}\right|^{2}\right\rangle_{*}
\end{aligned}
$$




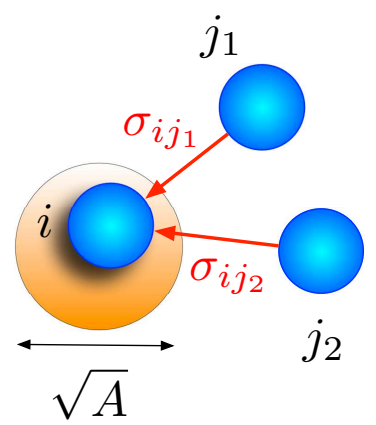

FIG. 7. Fluctuation of stresses due to fluctuations of particles inside a cage

In practice, it is useful to express various terms in Eq. (152) in terms of particle distributions so that standard techniques of the density functional theories of liquids [23] can be incorporated. The details of the technical aspects are presented in Appendix $\mathrm{E}$ F and $\mathrm{G}$

Let us discuss the two terms on the r. h. s. of Eq. (153) below,

- Born term of the renormalized liquid

The terms $J_{2}$ and $J_{3}$ are corrections to the Born term due to the renormalization of the potential Eq. (94). It is easy to realize that the sum of the three terms, i. e. the Born term, $J_{2}$ and $J_{3}$ is equivalent to the Born term of the renormalized liquid with the effective potential Eq. (94),

$$
\left\langle b_{\left[\left\{\mathbf{R}_{i}\right\}\right]}^{\mathrm{eff}}\right\rangle_{T / m,\left[v_{\mathrm{eff}}(r)\right]}=\left\langle b_{\left[\left\{\mathbf{R}_{i}\right\}\right]}\right\rangle_{*}+(1-m)\left(J_{2}+J_{3}\right)+O\left(A^{2}\right)
$$

with $\langle\ldots\rangle_{T / m,\left[v_{\text {eff }}(r)\right]}$ defined in Eq. (95). Here $b_{\left[\left\{\mathbf{R}_{i}\right\}\right]}^{\mathrm{eff}}$ is the Born term of the renormalized liquid which can be obtained by substituting $v_{\text {eff }}(r)$ into $v(r)$ of Eq. (9).

- Non-affine correction

The term $J_{1}$ is clearly distinct from others. While the contributions of the terms $J_{2}$ and $J_{3}$ vanish above $T_{\mathrm{K}}$ where $m^{*}(T)=1$, the contribution of the term $J_{1}$ does not. Presumably it continues to exist up to $T_{\mathrm{c}}$ where the metastable solid states disappear. Note that it has the same structure as the generic intra-state susceptibility $\hat{\chi}$ (See Eq. (114) ) discussed in sec. [1C7, Let us rewrite the $J_{1}$ term as,

$$
m J_{1}=\frac{1}{\kappa_{\mathrm{eff}}} \frac{1}{N} \sum_{i}\left\langle\left|\boldsymbol{\Xi}_{i}\right|^{2}\right\rangle_{*}
$$

where $\kappa_{\text {eff }}$ is defined in Eq. (108) and $\boldsymbol{\Xi}$ is the derivative of the shear-stress introduced in Eq. (38). In [63] it is pointed out that the field $\boldsymbol{\Xi}_{i}$ defined in Eq. (38) is a random field with very short-ranged correlation in space.

The physical interpretation of this term is straightforward and quite suggestive. Consider time scales shorter than the $\alpha$-relaxation time, where the stress-field does not evolve much so that, as a first approximation, we can assume it is a frozen-in vector field associated with a metastable state. As shown schematically in Fig. 7, the fluctuation of a particle, say particle $i$, inside its cage induces fluctuations of the stresses between the particle and the surrounding particles, say $j_{1}$ and $j_{2}$. The point is that the resultant fluctuations of the stresses $\sigma_{i j_{1}}$ and $\sigma_{i j_{2}}$ are correlated since they originate from the common source of fluctuation, the fluctuation of particle $i$.

Although we have only performed the cage expansion up to 1st order, we expect that the structure Eq. (153) is generic. Higher order cage expansion will yield more accurate effective potential $v_{\text {eff }}(r)$ [20]. This will amount to refine the renormalized Born term Eq. (154) and the $J_{1}$ term. Similarly, the $\Xi$ field Eq. (38) will be replaced by that of renormalized potential at higher orders in the cage expansion.

It is instructive to compare the above result with that of the rigidity $\mu_{\mathrm{IS}}$ of inherent structures (IS) discussed in sec. IA 7 The comparison is essentially the same as the comparison of the susceptibility associated with inherent structures $\chi_{\mathrm{IS}}$ and the intra-state susceptibility $\hat{\chi}$ we discussed in $\amalg \mathrm{C} 8$. Comparing the full expression of the rigidity of inherent structures $\mu_{\mathrm{IS}}$ Eq. (37) with our results of the intra-state rigidity $\hat{\mu}$ Eq. (155) together with Eq. (147), we find that the our scheme approximate the full Hessian matrix by that of an effective Einstein model. The other, more important difference is that the reference state itself used in the evaluation of the intra-state susceptibility $\hat{\mu}$ is not completely frozen-in one of energy minima, at marked variance with that for $\mu_{\text {IS }}$. The reference state is just took 
from a thermalized liquid configuration in a metastable state. Then it is natural to expect that the amplitude of the $\Xi$ field $\left\langle\left|\boldsymbol{\Xi}_{i}\right|^{2}\right\rangle_{*}$, which appears in Eq. (155), is larger than that at inherent structures (See Eq. (40)). Thus we consider that the intra-state rigidity $\hat{\mu}$ obtained above is most likely smaller than the rigidity of inherent structure $\mu_{\mathrm{IS}}$ for the same reason as the generic intra-state susceptibility $\hat{\chi}$ is larger than the susceptibility $\chi_{\text {IS }}$ associated with inherent structures as we discussed in sec. II 8 8. We consider that the underlying physical processes are thermal excitations between different inherent structures within a common metabasin, which are probably localized plastic deformations as we discussed in sec. П A 8 .

Finally let us recall the discussion in sec $1 \mathrm{~B}$ where we pointed out that mean-field theories generically imply that the rigidity of solids is closely related to the order parameter, more precisely the rigidity is simply proportional to the square of the order parameter (see also Appendix. C for a corresponding problem in a certain vectorial spin-glasses) . Indeed the computation of the intra-state rigidity $\hat{\mu}$ of glasses performed in the present section is consistent to some extent with this generic picture. We found the intra-state rigidity $\hat{\mu}$ can be expanded in power series of the order parameter $A$ as Eq. (140) combined with Eq. (102)), which reads,

$$
\hat{\mu}(A)=c_{0}-c_{1} A+O\left(A^{2}\right)
$$

with $c_{0}$ and $c_{1}$ being positive constants related to $\hat{\mu}_{0}$ and $-\hat{\mu}_{1}$ respectively (See Eq. (144) and Eq. (150)). The above expression Eq. (156) implies larger cage size $A$ yields smaller rigidity $\hat{\mu}$. Here it is instructive to note that the equivalent of the cage size $A$ in the spin-glass case is $1-q$ with $q$ being the Edwards-Anderson order parameter as we point out in Appendix B (see also Appendix C).

\section{Fluctuation of the shape of the container : shear-strain fluctuations}

So far we have implicitly assumed that the shape of the container, parametrized by the shear-strain $\gamma$, is rigid and fixed. What will happen if we allow it to fluctuate? As long as the rigidity $\hat{\mu}$ is positive, we can safely integrate over the shear-strain $\gamma$ to obtain an elastic contribution to the free-energy of the cloned liquid. Using Eq. (153) we find,

$$
-\beta m \frac{F_{m}^{\text {elastic }}(\alpha)}{N}=\log \int \prod_{a} d \gamma^{a} e^{-\beta \sum_{a b} \frac{\mu_{a b}}{2} \gamma^{a} \gamma^{b}}=\log \sqrt{\frac{(2 \pi)^{m-1}}{m^{m-2}(\beta \hat{\mu}(\alpha) / m)^{m-1}}} .
$$

with

$$
\mu_{a b}=\hat{\mu}(\alpha)\left(\delta_{a b}-\frac{1}{m}\right)
$$

Here the intra-state rigidity $\hat{\mu}$ is given by Eq. (140),

$$
\hat{\mu}(\alpha)=\hat{\mu}_{0}+\hat{\mu}_{1} \alpha+O\left(\alpha^{2}\right)
$$

\section{First order}

Let us first discuss the consequence of the fluctuation of the shear-strain $\gamma$ at the 1st order in the cage expansion. By adding $F_{m}^{\text {elastic }}(\alpha)$ to the free-energy $F_{m}(\alpha)$ Eq. (97) and performing the Legendre transform Eq. (88) we find

$$
\alpha(A)=m A+\left(\frac{2 m \beta}{d} \frac{1}{N} \sum_{i<j}\left\langle\nabla^{2} v\left(r_{i j}\right)\right\rangle_{*}+\frac{m^{2}}{d} \frac{\hat{\mu}_{1}}{\hat{\mu}_{0}}\right) A^{2}+O\left(A^{3}\right)
$$

Then the free-energy $G_{m}(A)$ Eq. (101) is modified as,

$$
\frac{\beta}{N} G_{m}(A)=\frac{d(1-m)}{2 m} \log \left(2 \pi A / \Lambda^{2}\right)-A \frac{1-m}{m} \frac{1}{N} \sum_{i<j} \beta\left\langle\nabla^{2} v\left(r_{i j}\right)\right\rangle_{*}-\frac{1-m}{2 m} \log (\beta \hat{\mu}(\alpha(A)))+\ldots,
$$

where we omitted terms independent of the cage size $A$. Now the equation $0=\partial G_{m}(A) / \partial A$ by which the cage size is determined Eq. (103) is replaced by,

$$
0=\frac{1}{A}-\frac{2 \beta}{d} \frac{1}{N} \sum_{i<j}\left\langle\nabla^{2} v\left(r_{i j}\right)\right\rangle_{*}-\frac{\hat{\mu}_{1}}{\hat{\mu}_{0}} \frac{m}{d}+O(A)
$$


which yields,

$$
A=\frac{d}{2 \beta \frac{1}{N} \sum_{i<j}\left\langle\nabla^{2} v\left(r_{i j}\right)\right\rangle_{*}+m \hat{\mu}_{1} / \hat{\mu}_{0}} .
$$

Since $\hat{\mu}_{1}$ is negative (see Eq. (150) ) the result Eq. (163) means that the fluctuation of the container tends to increase the cage size and thus weakens the glassy order.

In turn, the increase of the cage size will make the rigidity smaller. Using Eq. (163) back in Eq. (160), we find again $\alpha=2 m A$, which is the same as Eq. (106). Thus the intra-state rigidity of the system which is contained in the deformable container becomes,

$$
\hat{\mu}=\hat{\mu}_{0}-2 m A \hat{\mu}_{1} .
$$

This is formally the same as the previous one Eq. (153) but it is now smaller than the latter because the cage size $A$ is increased as found above Eq. (163).

\section{Coupling of the cage size and the shear-strain fluctuations : a possible scenario}

An interesting feature found above is that fluctuation of the shear-strain is coupled to the cage size $A$, which is the order parameter of the glassy state, and makes it larger. We suspect that the actual effect of the shear-strain fluctuation would be much stronger than the behaviour found at the 1st order level. Let us discuss a possible consequence of the coupling below.

By fully taking into account the elastic free-energy beyond the 1st order cage expansion, the saddle point equation for the cage size $A$ Eq. (162) may be cast into the following simple form,

$$
0=\frac{1}{A}-\frac{1}{A_{0}}+\frac{r}{1-r \frac{A}{A_{0}}} \frac{1}{A_{0}}
$$

where $A_{0}$ is the cage size in the absence of the shear-strain fluctuation. We also introduced a dimensionless parameter $r$ which represents the strength of the non-affine correction,

$$
r \equiv-\frac{\hat{\mu}_{1}}{\hat{\mu}_{0}} m A_{0} .
$$

Note that $\hat{\mu}_{0}>0$ and $\hat{\mu}_{1}<0$ (see Eq. (144) and Eq. (150) so that $r$ is a positive parameter. We anticipate that it is an increasing function of the temperature $T$. Although we have not performed higher order cage expansions explicitly, we expect that the structure of Eq. (165) is generic. It can be simplified as,

$$
a=\frac{1}{1-r a} .
$$

where

$$
a \equiv \frac{A}{A_{0}}
$$

The solution of Eq. (167) is easily found as,

$$
a=\frac{1-\sqrt{1-4 r}}{2 r}=1+r+O\left(r^{2}\right)
$$

where we have took the physical solution which becomes 1 in the limit $r \rightarrow 0$. Most interesting feature is that the cage size exhibits a square-root singularity approaching a critical point,

$$
a \simeq a_{c}\left(1-\sqrt{\frac{r}{r_{c}}-1}\right) \quad\left(r \rightarrow r_{c}^{-}\right),
$$

with $r_{c}=1 / 4$ and $a_{c}=2$ and disappears at $r_{c}$. Correspondingly this is reflected on the intra-state rigidity as,

$$
\hat{\mu} \simeq \hat{\mu}_{c}\left(1+\sqrt{\frac{r}{r_{c}}-1}\right) \quad\left(r \rightarrow r_{c}^{-}\right)
$$


where $\hat{\mu}_{c}=\hat{\mu}_{0} / 2$. Thus the rigidity exhibits a discontinuous jump preceded by a square-root singularity.

The above observations imply the consequence of the coupling between the order parameter and the strain fluctuation can be significant leading to collapse of metastable solid states when the strength of non-affinity $r$ become strong enough. Interestingly enough the the discontinuous vanishment of the rigidity preceded by the square-root behaviour Eq. (171) is apparently similar to the expectation in the pure mean-field limit discussed in sec. IIB. However here the rigidity is not merely subjected to the order parameter at variance to the pure mean-field scenario but plays an essential role for the melting, partly supporting the intuition of Born's rigidity crisis scenario [37].

\section{E. Discussion: how the rigidity disappears?}

An interesting general question is how the rigidity of the metastable solids disappear by raising the temperature. Of course glasses melt at the glass transition temperature $T_{\mathrm{g}}$. But the effective rigidity $\hat{\mu}$ remains finite in the supercooled liquid state above $T_{\mathrm{g}}$ so that a supercooled liquid can be viewed as a solid with finite life time. Our interest is how the rigidity of this temporally solid disappear by increasing temperature.

\section{Mean-field theories}

In sec. IIB we pointed out that mean-field theories generically imply that the rigidify of solid states are simply proportional to the square of the order parameters. Then it immediately follows that the rigidity vanish discontinuously signaled by preceding square-root behaviours approaching spinodal like melting temperatures from below, simply reflecting the same behaviour of the order parameters. We showed that this scenario holds in a Ginzburg-Landau theory for superheated ferromagnet (sec. IIB 1), the mode coupling theory for supercooled liquids (sec. IIB 2 ) and a replica field theory for vectorial spin-glasses (Appendix. C).

In sec IIIC we performed a microscopic computation of the rigidity of supercooled liquids and glasses based on the cloned liquid theory. Indeed the result also suggests that the rigidity is intimately related to the order parameter $A$ as Eq. (156), which reads as,

$$
\hat{\mu}(A)=c_{0}-c_{1} A+O\left(A^{2}\right) .
$$

with $c_{0}$ and $c_{1}$ being certain positive constants. Since both the mode coupling theory [11, 12] and the cloned liquid theory [20] predict that the cage size $A$ exhibits a discontinuous jump preceded by a square-root behaviour $A\left(T_{\mathrm{c}}\right)-A(T) \propto \sqrt{T_{\mathrm{c}}-T}$ as $T \rightarrow T_{\mathrm{c}}^{-}$, the rigidity $\hat{\mu}$ as given by Eq. (172) should also exhibit a discontinuous jump at $T_{\mathrm{c}}$ preceded by a square root singularity, at least at the mean-field level. Let us note that a recent formulation of a replica approach to the rigidity of glasses by Szamel and Flenner [103] is consistent with this scenario.

To summarize, at the pure mean-field level, the rigidity does behave as the order parameter. Said differently, at this level the transverse fluctuations such as shear-strains, spin-waves, e.t.c. do not play any significant roles compared with the longitudinal fluctuations such as the fluctuation of the density, amplitude of vectorial order parameters, e.t.c. because the rigidity is totally subjected to the order parameter which parametrize the strength of the longitudinal fluctuations. The situation may change if we consider explicitly influences of the transverse fluctuations going beyond the pure mean-field level as we discuss below.

\section{Influence of transverse fluctuations}

In sec IIID, we discussed an alternative melting mechanism due to coupling between the order parameter, i. e. the cage size $A$ and fluctuations of the shape of the container, i. e. shear-strain. Interestingly enough it also predicts discontinuous jumps of the cage size $A$ and the rigidity $\hat{\mu}$ with square-root singularities. However the driving mechanism of the melting is different from the one discussed above. In this case the control parameter is the nonaffinity $r$ defined in Eq. (166) which is presumably an increasing function of the temperature $T$.

At the pure mean-field level, fluctuation of the shear-strain can be forgotten by just considering a container with a fixed shape. However we may view a finite dimensional system as an assembly of deformable cells for each of which we can define an effective mean-field free-energy. Such a local mean-field free energy on a cell around a point $\mathbf{r}$ may be expressed in terms of the local order parameter $A(\mathbf{r})$ and the shape of the cell parametrized by local shear-strain $\gamma(\mathbf{r})$. In this description the shear-strain $\gamma(\mathbf{r})$ naturally becomes a fluctuating continuous field (elastic strain field) which we must integrate out.

The equivalent of the shear-strain field $\gamma(\mathbf{r})$ in spin systems are the derivative of the angular field $\nabla \theta(\mathbf{r})$ which parametrizes the spin-waves discussed in sec ЏB1 (ferromagnets) and Appendix C (spin-glasses). Apparently the 
the spin-wave $\nabla \theta(\mathbf{r})$ must be integrated out in order to take into account fluctuations around the mean-field theories. As the result we will obtain certain renormalized effective potential for the longitudinal fluctuations, which should be analogous to the one we discussed in sec. IIID.

However, as long as the effects of the transverse fluctuations can be took into account perturbatively, the overall basic characters of the spinodal like criticality themselves would remain intact. It will be very interesting to examine possible non-perturbative effects of the transverse fluctuations in future.

\section{Comparison with related problems}

The spinodal like criticality is very different from the case of usual 2 nd order phase transitions (e.g. $\phi^{4}$ theory) where the order parameter behaves continuously at the transition point so that the rigidity also exhibits a continuous behaviour across the transition temperature as we noted in the end of sec. II B 1. In the latter case the vanishing of the rigidity is related to the divergence of static correlation length at the transition through the so called Josephson's scaling relation [52]. Such continuous behavior of the order parameter and the rigidity have been predicted for the cases of spin-glass transitions [104] and vulcanization transitions [44 46].

Jamming transition is quite intriguing in this respect because it cannot be understood simply along the line of thoughts discussed above: some simplified model systems for granular matters exhibit discontinuous jump of the contact number preceded by a square-root singularity but continuous behaviour of the rigidity at the (un) jamming transition [48, 53, 105].

\section{F. A model computation - binary soft sphere system}

We now perform an explicit computation of the rigidity for the case of a binary mixture of soft-spheres in three dimensions [106], which is a standard model system to study the supercooled liquid state and the glassy state. We compare our results with the rigidity of the same system obtained by Barrat et. al. 36 using molecular dynamic (MD) simulations. Thermodynamic properties of the same system has been analyzed using the cloned liquid theory by Coluzzi et. al. [19].

\section{Model}

The system consists of two kinds of particles $\epsilon=+,-$ which have different radius $\sigma_{+}$and $\sigma_{-}$and interact with each other by a repulsive potential,

$$
v^{\epsilon_{i} \epsilon_{j}}\left(r_{i j}\right)=u_{0}\left(\frac{\sigma_{\epsilon_{i}}+\sigma_{\epsilon_{j}}}{r_{i j}}\right)^{12} .
$$

We introduce a smooth long-range cut-off of the potential following [43] which endures continuity up to the 2nd derivative of the potential. We choose the number fractions of the two types of particles as $x_{+}=x_{-}=1 / 2$ and the choose the ratio of the diameters as $\sigma_{+} / \sigma_{-}=1.2$ [36, 107, 108]. The radii $\sigma_{+}$and $\sigma_{-}$are fixed by requiring that the effective diameter to be equal to the unit length scale $l_{0}$, i. e. $\left(\sum_{\epsilon= \pm, \epsilon^{\prime}= \pm} x_{\epsilon} x_{\epsilon^{\prime}}\left(\sigma_{\epsilon}+\sigma_{\epsilon^{\prime}}\right)^{3}\right)^{1 / 3}=l_{0}$. Then the thermodynamic properties are determined by a single parameter $\Gamma=\rho l_{0}^{3}\left(k_{\mathrm{B}} T / u_{0}\right)^{-1 / 4}$. Here $\rho=N / V$ is the number density where $V$ is the volume of the system measured in the unit of $l_{0}^{d}$. In the following we choose $\rho=1$ and study the temperature range $0.05<T<0.3$ where the temperature is measured in the unit of $u_{0} / k_{\mathrm{B}}$. The mode coupling critical temperature $T_{\mathrm{c}}$ and Kauzmann transition temperature $T_{\mathrm{K}}$ of the system has been found to be $T_{\mathrm{c}} \sim 0.19-0.22$ by molecular dynamics simulations [107] [109] and $T_{\mathrm{K}} \simeq 0.125$ by the cloned liquid theory [19].

\section{Analysis}

Our analysis proceeds as follows. First we obtain some basic thermodynamic parameters. To this end we analyzed the thermodynamics of the system following the scheme by Coluzzi et. al. in [19]. More precisely we used the 1st order cage expansion for the binary system which is a straight forward extension of the prescription [18] summarized in sec. II C5. We employed the binary Hyper Netted Chain (HNC) approximation [19], which is the HNC approximation [110] adapted to the binary mixture, to obtain the radial distribution functions $g^{\epsilon \epsilon^{\prime}}(r)$ between particles of type $\epsilon= \pm$ 


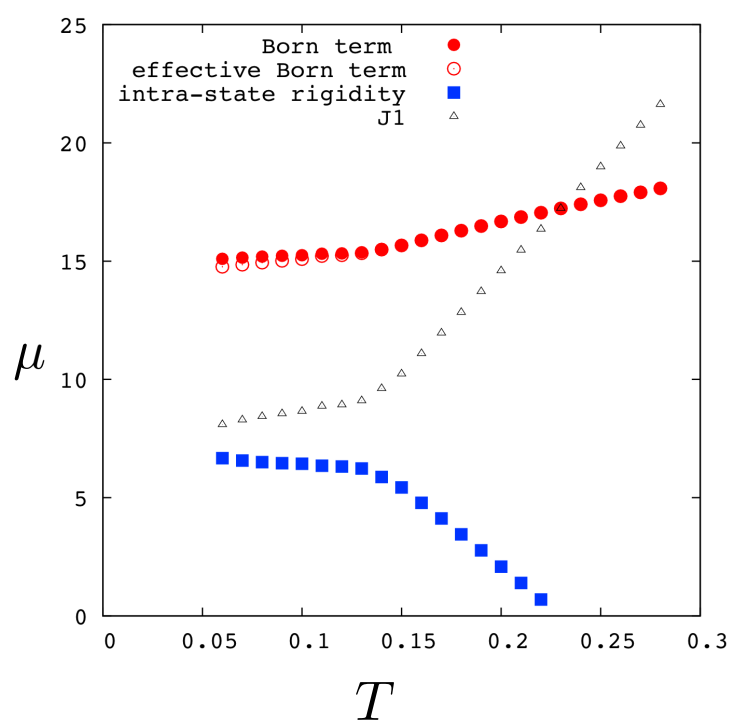

FIG. 8. Rigidity of a binary soft-sphere system, computed by the cloned liquid approach. The Kauzmann temperature of the system is $T_{\mathrm{K}} \sim 0.12[19]$ and the mode coupling critical temperature is around $T_{\mathrm{c}} \sim 0.19-0.22$ [107] 109]. The intra-state rigidity $\hat{\mu}$ (squares) is nearly independent of temperature below $T_{\mathrm{K}}$ while it sharply decreases with increasing temperature above $T_{\mathrm{K}}$. On the other hand, the instantaneous rigidity, i. e. the Born term (filled circles) increases with the temperature. The renormalization of the Born term is very weak (open circles). The significant part of the non-affine correction is due to the $J_{1}$ term (triangles), whose magnitude sharply increases with temperature above $T_{\mathrm{K}}$.

and $\epsilon^{\prime}= \pm$ of the liquid state of the binary system, and the free-energy of the liquid state Eq. (98) which are needed in the thermodynamic analysis. As the main outcome of the thermodynamic analysis, we obtain radial distribution functions $g^{\epsilon \epsilon^{\prime}}(r)$, the the glass-transition line $m=m^{*}(T)$ and the cage sizes $A_{\epsilon}(T)$ of the two kinds of particles $\epsilon= \pm$ which are indispensable in the following analysis.

Next we compute the intra-state rigidity $\hat{\mu}$ of the system at the level of 1 st order cage expansion. To this end we need to evaluate various terms in Eq. (152) by representing them using particle distribution functions as explained in Appendix E, F and G. The values of the parameters $c_{\epsilon}$ Eq. (G4), which controls the strength of the non-affine corrections, are obtained using the cage sizes $A_{\epsilon}$ and the parameter $m^{*}(T)$ determined in the thermodynamic analysis. We use the radial distribution functions $g^{\epsilon \epsilon^{\prime}}(r)$ in the evaluation of the Born term Eq. (G2) and the non-affine correction term $J_{2}$ Eq. (G3). All integrals $\int d r \ldots$ are done numerically. To evaluate the non-affine correction term $J_{1}$, which involves three-point particle distribution function (see Eq. (E12)), we use the Kirkwood superposition approximation Eq. (E5) which approximates the three-point function as a product of two-point functions $g^{\epsilon \epsilon^{\prime}}(r)$ (See Eq. (G10) and Eq. (G11)). To evaluate the non-affine correction term $J_{3}$ Eq. (G5), we need to evaluate the derivatives $\partial g_{*}^{\epsilon^{\prime} \epsilon^{\prime \prime}}\left(r ; \delta_{+}, \delta_{-}\right) /\left.\partial \delta_{\epsilon}\right|_{\delta=0}$. In practice, we obtain them as follows: first we evaluate the radial distribution functions $g^{\epsilon^{\prime} \epsilon^{\prime \prime}}(r)$ using the binary HNC [19] for the modified potential Eq. [G6) with some sufficiently small values of the perturbation $\delta_{\epsilon}$ and then we take numerical derivatives of the results with respect to $\delta_{\epsilon}$.

\section{Results}

As shown in Fig. 8, the intra-state shear-modulus $\hat{\mu}$ exhibits the following characteristic temperature dependence. On one hand it is almost independent of the temperature below the Kauzmann transition temperature $T_{\mathrm{K}} \sim 0.12$ and takes a value around $\hat{\mu} \sim 6$. On the other hand it becomes strongly dependent on the temperature at $T>T_{\mathrm{K}}$. Evidently there is a significant difference between the Born term, which represents the affine part of the response, and the intra-state rigidity $\hat{\mu}$ at all temperatures. Even at very low temperatures the reduction of the rigidity due to non-affine corrections is significant, in agreement with numerical studies of static response to shear of energy minima (inherent structures) 62, 63]. Moreover the sharp decrease of $\hat{\mu}$ above $T_{\mathrm{K}}$ is clearly due to the non-affine correction. The fact that the Born term $b$ increases with increasing temperature would appear somewhat puzzling but it simply 
a)

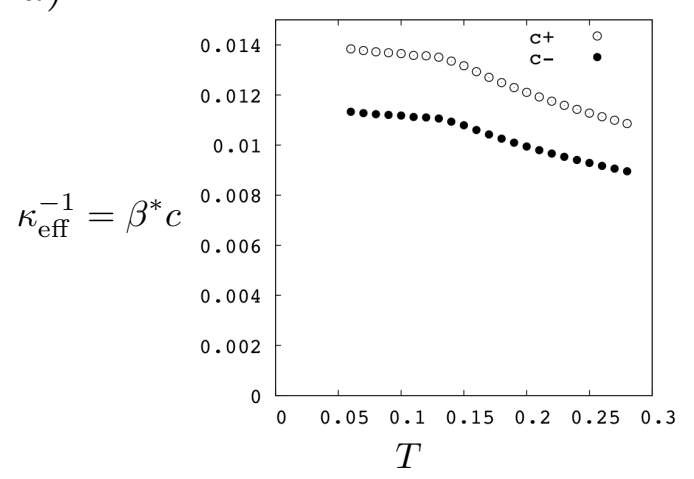

b)

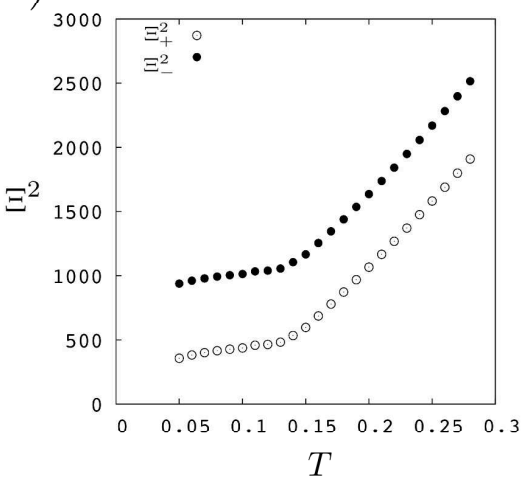

FIG. 9. Two temperature dependent factors in the non-affine correction term $J_{1}$. In the binary system the $J_{1}$ term Eq. (155) can be written as (see Eq. (G7) ), $m J_{1}=\sum_{\epsilon= \pm} c_{\epsilon} \beta^{*} \Xi_{\epsilon}^{2}$. The panel a) shows the inverse of the effective Hookian spring constants $\kappa_{\epsilon}^{-1}=\beta^{*} c_{\epsilon}$ with $c_{\epsilon}$ as defined in Eq. (G4), multiplied by $\beta^{*}=m^{*}(T) \beta$. The panel b) shows the squared amplitudes of the $\boldsymbol{\Xi}$ fields, $\Xi_{\epsilon}^{2}$ as defined in Eq. G8.

means that particles, which are interacting with each other by purely repulsive interactions, are colliding each other more often at higher temperatures so that instantaneous increase of the energy (without any relaxation) with a given shear-strain $\gamma$ becomes larger at higher temperatures.

Let us examine the non-affine corrections in more detail. As discussed in sec. ЩII C3, the non-affine corrections can be divided into two groups: 1$)$ the group of terms $\left(J_{2}\right.$ and $\left.J_{3}\right)$ which yields the renormalized born term $b^{\text {eff }}$ Eq. (154) together with the original born term $b$ and 2) the $J_{1}$ term Eq. (155) which is distinct from others. In Fig. 8 we included the curves of both the renormalized born term $b^{\text {eff }}$ and the $J_{1}$ term. Apparently the major contribution to the non-affine correction is the $J_{1}$ term. As can be seen in Fig. 8 it only weakly depends on the temperature at $T<T_{\mathrm{K}}$ but sharply increases with the temperature above $T_{\mathrm{K}}$.

From the definition Eq. (155) (more precisely Eq. (G7) which is adapted for the binary case), it can be seen that there are two different sources of the temperature dependence of the $J_{1}$ term. One is the factors $\kappa_{\epsilon}^{-1}=c_{\epsilon} \beta^{*}$ which are the inverse of the effective Hookian spring constants (see Eq. (147) and Eq. (108)) and the other is the amplitudes of the $\Xi$ fields. As shown in Fig. 9, the main source of the strong temperature dependence of the non-affine correction term above $T_{\mathrm{K}}$ is clearly the amplitude of the $\Xi$ field. We have argued in sec. II A 8 and sec. III C3 that the non-affine correction term can explicitly depend on the temperature because it reflects thermal fluctuations within metastable states (metabasins).

\section{Discussions}

We found the intra-state shear-modulus $\hat{\mu}$ becomes almost independent of the temperature below $T_{\mathrm{K}}$ suggesting that thermal fluctuations are significantly suppressed at $T<T_{\mathrm{K}}$. Then the value of $\hat{\mu} \sim 6$ at $T<T_{\mathrm{K}}$ may be compared with the rigidity of inherent structures $\mu_{\text {IS }}$ (see Eq. (50)). Quite interestingly an analysis of $\mu_{\text {IS }}$ Eq. (37) of the same system indicates $\mu_{\mathrm{IS}} \sim 5[111$ independently of the temperatures. Similarly it may also be compared with the onset value of the plateau $G_{\infty}$ in the stress relaxation (see Fig. 2). Indeed a molecular dynamic (MD) simulation of the same system indicates $G_{\infty} \sim 5[70]$ independently of the temperatures.

On the other hand, we found the intra-state shear-modulus $\hat{\mu}$ becomes smaller at higher temperatures at $T>T_{\mathrm{K}}$ (See Fig. 10). This is very different from both $\mu_{\mathrm{IS}}$ [111] and $G_{\infty}$ [69, 70, 112] which appear to depend little on the temperature up to surprisingly much higher temperatures. Thus we expect $G_{\mathrm{p}} \sim \hat{\mu}$ lies below $G_{\infty}$ at $T>T_{\mathrm{K}}$. However, somewhat puzzlingly, in numerical observations of stress-stress auto-correlation function $C_{\sigma}(\tau)$ [69, 70, 112] there are no indications of the existence of such an rigidity $G_{\mathrm{p}}$ lying below $G_{\infty}$. Probably this means that the distinction between the $\beta$-relaxation and the $\alpha$-relaxation is just a smooth crossover in the real dynamics at variance with the idealized picture shown in Fig. 21).

In Fig. 10 we compare the intra-state rigidity $\hat{\mu}$ with the static rigidity obtained by a previous MD simulation on exactly the same system done by Barrat et. al. (1988) [36]. In the MD simulation [36] the static fluctuation formula for the elastic modulus (see sec. IIA 2) was used to compute the elastic moduli. It appears that the theoretical curve, which is obtained with no adjustable parameters, compares reasonably well with the data points of the MD simulation. However we should note that this comparison is delicate since static rigidity of a truly equilibrated system 


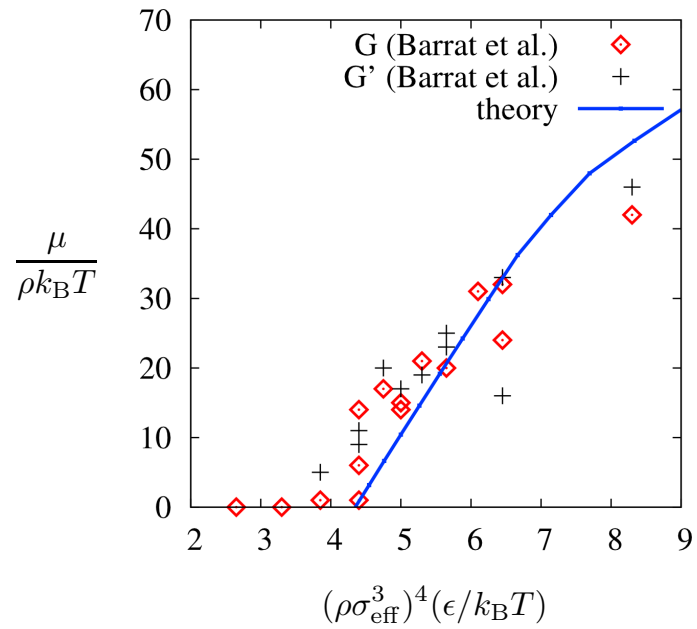

FIG. 10. Comparison of the rigidity of a binary soft-sphere system computed by Barrat et. al. (1988) 36$]$ with $N=3456$ and the theoretical one. Here $G$ and $G^{\prime}$ represent respectively the rhombohedral and tetragonal shear-modulus which become identical in isotropic elastic systems.

of large enough system sizes should become 0 at least down to $T_{\mathrm{K}} \sim 0.12$ (corresponding to $\left(\rho \sigma_{\mathrm{eff}}^{3}\right)^{4}\left(\epsilon / k_{\mathrm{B}} T\right) \sim 8.3$ in Fig. 10).

It is interesting to note that the intra-state shear-modulus $\hat{\mu}$, as well as the rigidity observed by the MD simulation [36], crosses 0 at around $T \sim 0.23$ (corresponding to $\left(\rho \sigma_{\text {eff }}^{3}\right)^{4}\left(\epsilon / k_{\mathrm{B}} T\right) \sim 4.3 \mathrm{in} \mathrm{Fig.} \mathrm{10),} \mathrm{which} \mathrm{is} \mathrm{rather} \mathrm{close} \mathrm{to} \mathrm{the}$ putative mode coupling critical temperature $T_{\mathrm{c}} \sim 0.19-0.22$ estimated by MD simulations [107] 109]. This feature may be interpreted in favor of the Born's original criteria for melting [37], i. e. continuous vanishing of the rigidity approaching the spinodal temperature is valid for the present system. The situation is similar to the numerical observations of the rigidity by some MD simulations on superheated crystals [39, 40]. However we note that our computation is limited to the 1st order cage expansion and does not tell us the location of the putative spinodal temperature where the glassy metastable states actually disappear.

The discussion in sec IIIEsuggests rather that the system would actually exhibit discontinuous melting but preceded by a continuous, square-root behavior of both the cage size $A$ and the intra-state rigidity $\hat{\mu}$ at a somewhat lower temperature. Then the seemingly continuous vanishing of the shear-modulus $\hat{\mu}$ around $T_{\mathrm{c}}$ is a pure coincidence and a small discontinuity may be hidden in the numerical data of [36] shown in Fig. 10. We have discussed two qualitatively different driving mechanisms for such a behavior. The strong temperature dependence of the non-affine correction term $J_{1}$ shown in Fig. 8 and the fairly good comparison of $\hat{\mu}$ with the data of the MD simulation shown in Fig. 10 suggest that the coupling between the cage size and the shear-strain fluctuation may be important in the present system. In this respect it is interesting to note that the conventional mode coupling theory which does not take into account the coupling of the shear-strain fluctuation and the density predicts a too large critical temperature $T_{\mathrm{c}} \sim 0.33$ [113]. However we must remind ourselves once more that melting of amorphous solids can be at most just a smooth crossover in finite dimensional systems.

\section{DISCUSSIONS}

Before concluding this paper, let us present discussions on some problems.

- In the present paper we analyzed static rigidity of the amorphous metastable states of structural glasses. We discussed a possible interpretation of the hierarchy of the static rigidities in the context of rheology in sec. IA5 - sec. II A 8, In particular, we argued that the rigidity of the inherent structures $\mu_{\mathrm{IS}}$ and the intra-state rigidity $\hat{\mu}$ correspond respectively to the rigidity at the onset of the plateau regime $G_{\infty}$ and the large time limit of the plateau regime $G_{\mathrm{p}}$ (see Fig. 2). However the plateaus become obscure at higher temperatures and it becomes difficult to separate the $\beta$ and $\alpha$-relaxations. A possible way out of this difficulty is to perform the analysis of fluctuation-dissipation relation (FDR) discussed in Appendix A2 70] which allows one to "eliminate times" and focus on the breaking point of the FDR, which is expected to correspond to the crossover point between the $\beta$ and $\alpha$ relaxations, i. e. $G_{\mathrm{p}}$. 
- From a broader perspective, the intra-state rigidity $\hat{\mu}$ is just one of many intra-state susceptibilities which characterize quasi-equilibrium material properties of amorphous solids, which are well accessible to experimental observations and important in practical use of amorphous solids. It is interesting to note that properties of the underlying "ground state" of a supercooled liquid can show up in the intra-state susceptibilities even if the system as a whole is out of equilibrium. As we discussed in sec. IIC 4 and in sec. II C 7 the cloned liquid approach realises decomposition of a generic susceptibility into intra-state ( $\beta$-relaxation) and inter-state ( $\alpha$-relaxation). This suggests possibilities to make further progress in understanding material properties of amorphous solids out of equilibrium starting from microscopic Hamiltonians.

- A natural step to go beyond the mean-field theoretical level is to consider spatial fluctuations of the order parameter i. e. the cage size $A(\mathbf{r})$, shear-strain $\gamma(\mathbf{r})$ and e.t.c. In particular we expect that the coupling of the shear-strain $\gamma(\mathbf{r})$ and the cage size $A(\mathbf{r})$ becomes important in finite dimensional systems as we discussed in sec. IIID and sec. IIIE Following the discussion in sec. [IIE, we may write a phenomenological Ginzburg-Landau like free-energy functional of the cloned liquid as,

$$
F[A(\mathbf{r}), \gamma(\mathbf{r})]=\int d^{d} r\left[\sum_{a b} \frac{\rho \hat{\mu}(A(\mathbf{r}))}{2}\left(\delta_{a b}-\frac{1}{m}\right) \gamma^{a}(\mathbf{r}) \gamma^{b}(\mathbf{r})+\frac{c}{2}(\nabla A(\mathbf{r}))^{2}+g_{m}(A(\mathbf{r}))\right]
$$

where $\rho=N / V$ is the number density, $g_{m}(A)=\rho\left(G_{m}(A) / N\right)$ is the local 'mean-field' free-energy with $G_{m}(A)$ given in Eq. (161) and $\hat{\mu}(A)$ is the intra-state rigidity. This can be compared with the free-energy functional Eq. (C9) of the replica field theory of vectorial spin-glasses discussed in Appendix C where the equivalent of the shear-strain field $\gamma(\mathbf{r})$ is the derivative of the angular field $\nabla \theta(\mathbf{r})$ which parametrizes the spin-waves. The free-energy functional defines an "effective Debye model" for amorphous solids. To be completed the elastic free-energy associated with normal strain (compression) must also be added. In principle all key parameters which parametrizes the free-energy functional can be determined by microscopic computations using the meanfield theory (cloned liquid theory). Especially computation of the bulk modulus which parametrizes the elastic free-energy associated with the normal strains can be done in the same way as done for the shear-modulus $\hat{\mu}$ in the present paper. However, we must note that such a coarse-grained description cannot describe important non-Debye features in amorphous solids such as the boson peak and anomalous soft-modes around jamming point [47].

We found the rigidity depends on the cage size $A$ as Eq. (156) which reads as,

$$
\hat{\mu}(A)=c_{0}-c_{1} A+O\left(A^{2}\right) .
$$

This implies the local rigidity $\hat{\mu}(\mathbf{r})$ is smaller in the region where the local cage size $A(\mathbf{r})$ is larger. It might be related to the numerical observation of the elastic heterogeneity [114]. It will be very interesting to study more in detail the consequences of the coupling between the transverse fluctuation (shear-strain) and longitudinal fluctuation (cage size) in the static and dynamic properties of amorphous solids and supercooled liquids, such as the dynamic heterogeneity [69, 115 118].

- We argued that inter-state part of the response to quasi-static shear brings about jerky, intermittent stressstrain curves at mesoscopic scales (see sec. IIIB). They are signatures of the inter-state fluctuations which are Goldstone modes in glasses which try to restore the broken the translational symmetry. It will be very interesting to perform some micro-rheological experiment [119] of slow strain rate in supercooled liquids to look for some signatures of the putative quasi-static intermittency. A possible gedanken experiment may be to slowly drag a single particle immersed in a deeply supercooled liquid through a Hookian spring attached it. Such an experiment can be realized for instance by applying magnetic field to a magnetic particle immersed in a colloidal glass system [119]. In order to suppress melting, the system may be confined in a narrow region between two walls with separation $L$ comparable or smaller than the mosaic length $\xi_{\text {mosaic }}$ [7, 9]. Then the interior of the local region (mosaic) around the particle will behave as a bulk system in the glass phase $T<T_{\mathrm{K}}$. By dragging the end of the spring slowly over some a small distance $x$, this region will be subjected to a local shear-strain of order $\delta \gamma \propto x / \xi_{\text {mosaic }}$. Then the force curve $F(x)$ of the spring will exhibit intermittent profiles either due to level crossings among different metastable states within the mosaic or yielding due to saddle-node bifurcation at metastability limits [32, 33]. A useful observable may be the force-force correlation function $C_{F}(x) \equiv \overline{F(0) F(x)}$ (where $\cdots$ means averaging over the space or trajectories). Presumably the correlation function $C_{F}(x)$ exhibit a cusp like singularity at $x=0$ (but rounded over some width $\delta x_{\mathrm{w}} \propto T$ at finite temperatures) and decays rapidly as function of $x / \delta x_{\mathrm{s}}$ where $\delta x_{\mathrm{s}}$ is the typical spacing between the shocks. The conjecture Eq. (133) implies $\delta x_{\mathrm{s}} \sim \xi_{\text {mosaic }} \delta \gamma_{\mathrm{s}} \sim \xi_{\text {mosaic }}^{1-d / 2}$. 


\section{CONCLUSIONS}

In this paper we developed a first principle scheme to evaluate the rigidity of supercooled liquids and glasses by analyzing the rigidity of a cloned liquid, which might appear absurd because the rigidity of a liquid is just zero. We showed that the replica technique allows one to switch-on or off contributions of the inter-state fluctuations of the shearstresses to find non-zero rigidity of amorphous metastable solids and inter-state responses with intermittent nature. We discussed physical interpretation of the result within the context of linear rheology and the phenomenological free-energy landscape picture. We also presented a model computation of the rigidity of the binary soft-spheres and found that the result compares well with the result of a previous molecular dynamic simulation. A strong advantage of the cloned liquid approach is that it fully makes use of conventional liquid theories such that one can choose the best one for a given problem at hand. Since the rigidity is a basic property which is very important both to understand the physics of glassy systems and to use amorphous materials in practice, it will be certainly interesting to develop the method further and apply it in various glasses and jammed matters.

Acknowledgment The author is grateful to Marc Mézard for the collaboration at the early stage of the present work and for many helpful discussions in the course of the present work. He thanks Giulio Biroli, Jean-Philippe Bouchaud, Peter Harrowell, Anaël Lemaître, Satoshi Okamura and Grzegorz Szamel for constructive comments and discussions. He thanks Jean-Louis Barrat, Ludovic Berthier, Andrea Cavagna, Jeppe C. Dyre, Silvio Franz, Akira Furukawa, Hikaru Kawamura, Jorge Kurchan, Florent Krzakala, Kunimasa Miyazaki, Tommaso Rizzo, Anne Tanguy, Eric Vincent and Francesco Zamponi for useful discussions. The author thanks LPTMS University of Paris Sud and SPEC CEA Saclay where some part of the present work has been done and for kind hospitality. This work is supported by a Triangle de la physique grant number 117 "Intermittent response of glassy systems at mesoscopic scales" and JPS Core-to-Core Program "International research network for non-equilibrium dynamics of soft matter".

\section{Appendix A: Fluctuation formula for stress relaxation}

\section{In equilibrium}

Here we outline a simple derivation of the generic fluctuation formula for the stress relaxation Eq. (11) [54]. To be specific we consider the time evolution of the system in terms of Liouville equation of motion,

$$
\left.\frac{d}{d t}|P(t)\rangle=\mathcal{L}|P(t)\rangle\right\rangle
$$

where vector $|P(t)\rangle\rangle$ represents probability distribution of the positions $\mathbf{r}_{i}$ and momentum $\mathbf{p}_{i}$ of particles $(i=$ $1,2, \ldots, N)$ and $\mathcal{L}$ is the Liouville operator;

$$
\mathcal{L}=\prod_{i=1}^{N}\left(-\frac{\mathbf{p}_{i}}{m} \cdot \frac{\partial}{\partial \mathbf{r}_{i}}+\frac{d U}{d \mathbf{r}_{i}} \cdot \frac{\partial}{\partial \mathbf{p}_{i}}\right) .
$$

The equilibrium distribution, which is an eigen vector of the Liouville operator with zero eigen value $\left.\mathcal{L}\left|P_{\text {eq }}\right\rangle\right\rangle=0$, is given by,

$$
\left.\left|P_{\mathrm{eq}}\right\rangle\right\rangle=Z^{-1} e^{-\beta \sum_{i=1}^{N} \frac{\left|\mathbf{p}_{i}\right|^{2}}{2 m}} e^{-\beta U},
$$

where $Z$ is the partition function Eq. (5).

The expectation value of the stress $\sigma$ observed at time $t$ can be written formally as,

$$
\langle\sigma(t)\rangle=\left\langle\left\langle\sigma e^{\mathcal{L} t} \mid P(0)\right\rangle\right\rangle
$$

where $\left\langle\left\langle\right.\right.$ on the r. h. s of the above equation represents traces over the positions $\mathbf{r}_{i}$ and momentum $\mathbf{p}_{i}$ of the particles. The stress is defined in Eq. (77) which reads as,

$$
\sigma=\left.\frac{1}{N} \frac{d U}{d \gamma}\right|_{\gamma=0}
$$

where $U$ is the potential part of the Hamiltonian of the system defined in Eq. (44). We assume that $U$ depends only on the positions $\mathbf{r}_{i}$ of the particles. 
The linear response of the stress with respect to infinitesimal changes of the strain $\delta \gamma(t)$ can be obtained formally as,

$$
\begin{aligned}
\langle\delta \sigma(t)\rangle & =\left\langle\left\langle\frac{d \sigma}{d \gamma} e^{\mathcal{L} t} \mid P(0)\right\rangle\right\rangle \delta \gamma(t)+\int_{0}^{t} d t^{\prime}\left\langle\left\langle\sigma e^{\mathcal{L}\left(t-t^{\prime}\right)} \frac{d \mathcal{L}}{d \gamma} e^{\mathcal{L} t^{\prime}} \mid P(0)\right\rangle\right\rangle \delta \gamma\left(t^{\prime}\right) \\
& =\langle b(t)\rangle \delta \gamma(t)+\int_{0}^{t} d t^{\prime}\left\langle\left\langle\sigma e^{\mathcal{L}\left(t-t^{\prime}\right)} \frac{d \mathcal{L}}{d \gamma} e^{\mathcal{L} t^{\prime}} \mid P(0)\right\rangle\right\rangle \delta \gamma\left(t^{\prime}\right)
\end{aligned}
$$

where

$$
b=\frac{d \sigma}{d \gamma}=\left.\frac{1}{N} \frac{d^{2} U}{d \gamma^{2}}\right|_{\gamma=0}
$$

is the Born term defined in Eq. (137) which represents the instantaneous, affine response to shear. The 2nd term on the r.h.s of Eq. A7 represents non-affine responses to shear due to stress relaxations.

If the system is equilibrated at the beginning, i. e. $\left.|P(0)\rangle\rangle=\left|P_{\text {eq }}\right\rangle\right\rangle$ we find the fluctuation formula Eq. (11),

$$
\langle\delta \sigma(t)\rangle=\langle b\rangle \delta \gamma(t)-\beta \int_{0}^{t} d t^{\prime} \frac{\partial C_{\sigma}\left(t, t^{\prime}\right)}{\partial t^{\prime}} \delta \gamma\left(t^{\prime}\right)
$$

where $C_{\sigma}\left(t, t^{\prime}\right)$ is the auto-correlation function of the stress,

$$
C_{\sigma}\left(t, t^{\prime}\right)=\left\langle\sigma(t) \sigma\left(t^{\prime}\right)\right\rangle=\left\langle\left\langle\sigma e^{\mathcal{L}\left(t-t^{\prime}\right)} \sigma e^{\mathcal{L} t^{\prime}} \mid P(0)\right\rangle\right\rangle
$$

Derivation of Eq. (A9) from Eq. (A7) is a standard one: one just need to notice,

$$
\left\langle\left\langle\ldots e^{\mathcal{L}\left(t-t^{\prime}\right)} \frac{d \mathcal{L}}{d \gamma} e^{\mathcal{L} t^{\prime}} \mid P_{\text {eq }}\right\rangle\right\rangle=\beta\left\langle\left\langle\ldots e^{\mathcal{L}\left(t-t^{\prime}\right)}\left(\mathcal{L} \frac{d U}{d \gamma}-\frac{d U}{d \gamma} \mathcal{L}\right) e^{\mathcal{L} t^{\prime}} \mid P_{\text {eq }}\right\rangle\right\rangle=-\beta \frac{\partial}{\partial t^{\prime}}\left\langle\left\langle\ldots e^{\mathcal{L}\left(t-t^{\prime}\right)} \frac{d U}{d \gamma} e^{\mathcal{L} t^{\prime}} \mid P_{\text {eq }}\right\rangle\right\rangle .
$$

Here the 2nd equation follows from the fact that $\left.\left|P_{\text {eq }}\right\rangle\right\rangle$ is the equilibrium distribution given by Eq. (A3).

\section{Out-of-equilibrium}

In out-of equilibrium glasses, i. e. glasses under aging or driven by external forces, the equilibrium fluctuation formula Eq. A9 cannot be expected. However the linear response can still be written formally as,

$$
\langle\delta \sigma(t)\rangle=\langle b\rangle \delta \gamma(t)-\beta \int_{0}^{t} d t^{\prime} X\left(t, t^{\prime}\right) \frac{\partial C_{\sigma}\left(t, t^{\prime}\right)}{\partial t^{\prime}} \delta \gamma\left(t^{\prime}\right)
$$

by introducing an unknown factor $X\left(t, t^{\prime}\right)$ which is 1 in the case of equilibrium response.

As suggested by the studies of the dynamical mean-field theory of spin-glasses [72], a natural way to investigate large time behaviors of out-of-equilibrium glassy systems is to consider large time limits $t, t^{\prime} \rightarrow \infty$ such that the value of the correlation function is fixed to a certain value $C_{\sigma}\left(t, t^{\prime}\right)=C$. In such a limit the $X$ factor becomes parametrized solely by the value of the correlation function $C$,

$$
\lim _{t^{\prime} \rightarrow \infty, C_{\sigma}\left(t, t^{\prime}\right)=C} X\left(t, t^{\prime}\right)=X(C)
$$

Then in the case of the typical stress relaxation protocol discussed in sec. IIA4 the linear response in the large time limits $t, t^{\prime} \rightarrow \infty$ with fixed $C=C_{\sigma}\left(t, t^{\prime}\right)$ can be written simply as,

$$
\lim _{t \rightarrow \infty, C_{\sigma}\left(t, t^{\prime}\right)=C} \frac{\langle\delta \sigma(t)\rangle}{\gamma}=\lim _{t \rightarrow \infty}\langle b(t)\rangle-\beta \int_{C}^{\lim _{t \rightarrow \infty} C_{\sigma}(t, t)} d C^{\prime} X\left(C^{\prime}\right)
$$

The simplest ansatz for the function $X(C)$ would be to assume that it consists of two pieces,

$$
X(C)= \begin{cases}1 & \left(\beta C>\mu_{\mathrm{p}} / x\right) \\ x & \left(\beta C<\mu_{\mathrm{p}} / x\right)\end{cases}
$$

This is exactly the case for the class of mean-field spin-glass (MFSG) models which exhibit 1 step replica symmetry breaking (RSB) including the $p$-spin spherical MFSG model [72, 90]. Because of the intimate analogy between the MFSG models which exhibit 1RSB and glass phenomenology [5 7, 9], it is often assumed that $X(C)$ function of structural glasses also has the form Eq. (A15). This ansatz is supported by numerical simulations 120 122. 


\section{Appendix B: Cloned liquid computation of the linear response of $p$-spin spherical mean-field spin-glass model}

Here let us analyze static magnetic linear response of the $p$-spin spherical mean-field spin-glass (MFSG) model using the cloned liquid approach. The validity of the cloned liquid approach for this system was demonstrated in [123]. Here we demonstrate that the decomposition of the intra-state and inter state linear magnetic susceptibility can be done exactly as discussed in sec. 【C4 and also by using the cage expansion approach discussed in sec. IIC7.

The Hamiltonian of the $p$-spin spherical MFSG model [124] is given by,

$$
H=-\sum_{1 \leq i_{1} \leq i_{2} \leq \ldots i_{p} \leq N} J_{i_{1} i_{2} \ldots i_{p}} s_{i_{1}} s_{i_{2}} \ldots s_{i_{p}}-h \sum_{i=1}^{N} s_{i}
$$

where the scalar spin variables $s_{i}$ at sites $i=1,2, \ldots, N$ Here we have introduced a uniform probing external magnetic field $h$. are forced to satisfy the spherical constraint $\sum_{i=1}^{N} s_{i}^{2}=N$. The random couplings $J_{i_{1} i_{2} \ldots i_{p}}$ are statistically independent from each other and drawn from a Gaussian distribution with zero mean and variance $J^{2} p ! /\left(2 N^{p-1}\right)$. In the case of $p \rightarrow \infty$ limit it reduced to the random energy model [92].

Let us consider a cloned system with $m$ replicas $a=1,2, \ldots, m$ with fixed overlap,

$$
\frac{1}{N} \sum_{i=1}^{N} s_{i}^{a} s_{i}^{b}=q \quad a \neq b .
$$

Static properties can be computed from the partition function of the cloned system, which is obtained as,

$$
Z_{m}\left(T, h^{a}\right)=\int \prod_{a, b} D \lambda_{a b} e^{-N\left(G\left(Q_{a, b}, \lambda_{a b}\right)+\delta G\left(\lambda_{a b}, h^{a}\right)\right)}
$$

where

$$
G\left(Q_{a b}, \lambda_{a b}\right)=-\frac{\beta^{2} J^{2}}{4} \sum_{a b} Q_{a b}^{p}-\sum_{a b} \lambda_{a b} Q_{a b}+\frac{1}{2} \log \left(\operatorname{det}\left(2 \lambda_{a b}\right) / \pi^{m}\right)
$$

with

$$
Q_{a b}=(1-q) \delta_{a b}+q
$$

and

$$
\delta G\left(\lambda_{a b}, h^{a}\right)=-\log \frac{1}{\sqrt{\pi^{m} / \operatorname{det}\left(2 \lambda_{\mathrm{ab}}\right)}} \int \prod_{a} D s^{a} e^{-\sum_{a b} \lambda_{a b} s^{a} s^{b}-\sum_{a} h^{a} s^{a}}=-\frac{1}{4} \sum_{a b} h^{a}\left(\lambda^{-1}\right)_{a b} h^{b}
$$

In Eq. (B3) we have simply took average of the partition function over disorder of the random couplings. This is the so called as annealed average which is valid only in the high temperature phase where replica symmetry is not broken. We analyze the low temperature region choosing small enough $m$ so that we remain in the high temperature phase

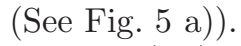

In Eq. (B3) we have introduced different probing field $h^{a}$ on different replicas. They are assumed to be infinitesimally small. Performing the integration over $\lambda_{a b}$ by the saddle point method ( at $h^{a}=0$ ) we find,

$$
\lim _{N \rightarrow \infty}-\beta m \frac{F_{m}\left(T, h^{a}\right)}{N}=-G\left(Q_{a b}, \lambda_{a b}^{*}\right)+\frac{1}{2} \sum_{a b} h^{a} Q_{a b} h^{b} \quad \lambda_{a b}^{*}=\frac{1}{2} Q_{a b}^{-1}
$$

This is formally the same as the 1 step replica symmetry breaking (RSB) solution [124]. Thus by performing extremization with respect to the parameters $q$ and $m$ one recovers the exact 1RSB free-energy of the system [123].

Using the above results the linear-susceptibility matrix $\chi_{a b}$ Eq. (80) is readily obtained as

$$
\chi_{a b}=\beta Q_{a b}=\beta(1-q) \delta_{a b}+\beta q .
$$

Comparing with Eq. (80) we can easily identify the intra-state linear susceptibility $\hat{\chi}=\beta(1-q)$ and inter-state susceptibility $\tilde{\chi}=m \beta q$. The total linear magnetic susceptibility becomes

$$
\chi=\sum_{b=1}^{m} \chi_{a b}=\beta(1-q)+m \beta q
$$


As expected these results agree with those of the usual 1 step RSB solution 124]. In the standard interpretation of the RSB solution [91] for the spin-glass phase below the static spin-glass transition temperature $T_{\mathrm{SG}}$, the factor $m$ (written rather as $x$ in the context of RSB in quenched disordered systems) in front of the 2nd term on the r. h. $\mathrm{s}$ is understood as the probability that two replicas stay at different metastable states. More physically the factor $m$ roughly corresponds to the probability that the system stay at an low lying 'excited' metastable state rather than 'ground' metastable state in the glass phase. The parameter $m$ is found to behave typically behaves as $m \sim T / T_{\mathrm{SG}}$ [92].

In the temperature range $T_{\mathrm{SG}}<T<T_{\mathrm{d}}$ (corresponding to $T_{\mathrm{K}}<T<T_{\mathrm{c}}$ ), the system in the liquid state in the thermodynamic sense. However the RSB solution Eq. (B9) is still very useful if we consider $m \rightarrow 1$ limit in the following sense. First note that the total susceptibility becomes $\lim _{m \rightarrow 1^{-}} \chi=\beta$ so that the correct paramagnetic susceptibility, which could be obtained by the replica symmetric (RS) ansatz which yields $q=0$, is recovered independently of the value of $q$. The value $q$ should be interpreted as self-overlap of metastable states which can be obtained as $\lim _{m \rightarrow 1^{-}} q$ using the RSB ansatz or equivalently the cloning liquid like methods [24, 83, 123]. Indeed the latter procedure correctly reproduce the self-overlap value $q$ of metastable states which dominates the thermodynamics of the "liquid state" at $T_{\mathrm{SG}}<T<T_{\mathrm{d}}$. Thus we can still decompose the total susceptibility into the intra-state susceptibility $\hat{\chi}=\beta\left(1-\lim _{m \rightarrow 1^{-}} q\right)$ and the inter-state susceptibility $\tilde{\chi}=\beta \lim _{m \rightarrow 1^{-}} q$ in this intermediate temperature range.

Now let us try the cage expansion approach to evaluate the linear susceptibilities as discussed in sec. II C7. To this end we decompose the spin variables into two parts as,

$$
s_{i}^{a}=S_{i}+\delta s_{i}^{a}
$$

where $S_{i}$ is the 'center of mass' of the spin

$$
S_{i}=\frac{1}{m} \sum_{a=1}^{m} s_{i}^{a}
$$

and $\delta s^{a}$ is the molecular coordinate which describes fluctuations around the 'center of mass'. As far as the spherical model is concerned, the effective action is already quadratic in terms of the spin variables (see Eq. (B6)) so that the decomposition Eq. (B11) is not particularly needed in order to compute the free-energy. Nonetheless, examination of the linear susceptibility by this approach may be instructive as we outline below.

The Hamiltonian of the cloned system can be written in terms of the new variables as,

$$
H_{m}=-\sum_{1 \leq i_{1} \leq i_{2} \leq \ldots i_{p} \leq N} J_{i_{1} i_{2} \ldots i_{p}} \sum_{a=1}^{m}\left(S_{i_{1}}+\delta s_{i_{1}}^{a}\right)\left(S_{i_{2}}+\delta s_{i_{2}}^{a}\right) \ldots\left(S_{i_{p}}+\delta s_{i_{p}}^{a}\right)-h \sum_{i} S_{i}-\sum_{a} \delta h^{a} \sum_{i} \delta s_{i}^{a}
$$

where we have introduced probing fields conjugated to the 'center of mass coordinate' $S_{i}$ and 'molecular coordinate' $\delta s_{i}^{a}$. Then we only need to replace Eq. (B6) ) with,

$$
\begin{aligned}
\delta G\left(\lambda_{a b}, h, \delta h^{a}\right)= & -\log \frac{1}{\sqrt{\pi^{m} / \operatorname{det}\left(2 \lambda_{\mathrm{ab}}\right)}} \int D S \prod_{a} D \delta s^{a} m \delta\left(\sum_{a} \delta s^{a}\right) e^{-\sum_{a b} \lambda_{a b}\left(S+\delta s^{a}\right)\left(S+\delta s^{b}\right)-h S-\sum_{a} \delta h^{a} \delta s^{a}} \\
& =-\frac{1+(m-1) q}{m} \frac{h^{2}}{2}+\frac{1}{2} \sum_{a b} \delta h^{a}\left(1-m \delta_{a b}\right) \frac{1-q}{m} \delta h^{b}
\end{aligned}
$$

Now we can readily find,

$$
\left\langle\delta s^{a} \delta s^{b}\right\rangle=-\left(1-m \delta_{a b}\right) \frac{1-q}{m} .
$$

This result is reminiscent of Eq. (92) combined with Eq. (102)). We can notice indeed that $1-q$ plays precisely the role of cage size $A$. Using the above results the linear-susceptibility matrix $\chi_{a b}$ can be obtained as,

$$
\chi_{a b}=\beta\left\langle s^{a} s^{b}\right\rangle=\beta\left\langle\left(s+\delta s^{a}\right)\left(s+\delta s^{a}\right)\right\rangle=\beta\left\langle S^{2}\right\rangle+\beta\left\langle\delta s^{a} \delta s^{b}\right\rangle=\beta\left[(1-q) \delta_{a b}+q\right] .
$$

The last result agrees with Eq. (B8) obtained without using the cage expansion.

\section{Appendix C: Rigidity of spin-waves in vectorial spin-glass systems}

Here we present a simple mean-field theoretical analysis of the rigidity of spin-waves in a class of vectorial spin-glass systems. Our purpose is to extend the analysis of the rigidity of spin-waves in the superheated ferromagnets discussed 
in sec. IIB 1 to the case of some spin-glass systems approaching the dynamical transition temperature $T_{d}$. To this end we consider a replica field theory with rotational invariance following Ref. [104] which analyzed the case of the Sherrington-Kirkpatrick model with vectorial spins.

Although the following discussion does not depend on the details of specific choices of microscopic models but we may consider as a specific example the $p$-spin spherical MFSG model Eq. (B1) extended to a model with vectorial spins,

$$
H=-\sum_{1 \leq i_{1} \leq i_{2} \leq \ldots i_{p} \leq N} J_{i_{1} i_{2} \ldots i_{p}}\left(\vec{s}_{i_{1}} \cdot \vec{s}_{i_{2}}\right)\left(\vec{s}_{i_{3}} \cdot \vec{s}_{i_{4}}\right) \ldots\left(\vec{s}_{i_{p-1}} \cdot \vec{s}_{i_{p}}\right) .
$$

The spin variables are two-component vectors $\vec{s}_{i}=\left(s_{i, 1}, s_{i, 2}\right)$ and the symbol - represents the inner product, i. e. $\vec{s}_{i} \cdot \vec{s}_{j} \equiv \sum_{\mu=1,2} s_{i, \mu} s_{j, \mu}$. Note that the model has the $O(2)$ symmetry just as the ferromagnetic system considered in sec. II B 1. The vectorial mean-field model can be solved by introducing a tensorial order parameter,

$$
Q_{a b}^{\mu \nu}=\frac{1}{N} \sum_{i=1}^{N}\left\langle S_{i, \mu}^{a} S_{i, \nu}^{b}\right\rangle
$$

where $\langle\ldots\rangle$ stands for thermal averages.

This class of systems with $p$ being an even integer greater than 2 , i. .e $p=4,6,8, \ldots$ exhibit the dynamical transition at $T_{\mathrm{d}}$ and static spin-glass transition at $T_{\mathrm{SG}}$ at a lower temperature just as the original scalar $p$-spin MFSG model [124 discussed in appendix B. The case of $p=2$ is the vectorial version of the Sherrington-Kirkpatrick model studied in [104] which exhibits the usual spin-glass transition at a single critical temperature.

To analyze generic properties of spin-waves in system as the one given above, let us consider a replica field theory of $m$ replicas $(a=1,2, \ldots, m)$ with a schematic free-energy functional,

$$
F\left[Q_{a b}^{\mu \nu}\right]=\int d^{d} r\left[\frac{c}{4} \sum_{a<b} \sum_{\mu \nu}\left(\nabla Q_{a b}^{\mu \nu}\right)^{2}+w\left(\sum_{\mu \nu}\left(Q_{a b}^{\mu \nu}\right)^{2}\right)\right] .
$$

Here the order parameter $Q_{a b}^{\mu \nu}(\mathbf{r})$ is considered as a continuous field in a $d$-dimensional space. Just as considered in appendix $\mathrm{B}$ we assume that this is a cloned system: replicas are forced to belong to a common equilibrium state. The local potential $w\left(\sum_{\mu \nu}\left(Q_{a b}^{\mu \nu}\right)^{2}\right)$ in the integrand of Eq. (C3) can be computed as the mean-field free-energy of the underlying microscopic model such as the one given by Eq. (C1) but the details are not important in the following discussion.

As noted in [104], an important symmetry property of the free-energy functional Eq. (C3) is that it is invariant under global rotations of any one (or more) of the replicas: it is invariant under a generic transformation of the form,

$$
Q_{a b}^{\mu \nu} \rightarrow \sum_{\mu^{\prime} \nu^{\prime}}\left(R^{t}\right)^{\mu \mu^{\prime}}\left(\theta_{a}\right) Q_{a b}^{\mu^{\prime} \nu^{\prime}} R^{\nu^{\prime} \nu}\left(\theta_{b}\right)
$$

where $\theta_{a}$ and $\theta_{b}$ are the angles of the global rotations of the replica $a$ and $b$ respectively. The rotations are represented by the rotational matrix $R^{t}(\theta)$,

$$
R^{t}(\theta) \equiv\left(\begin{array}{cc}
\cos (\theta) & -\sin (\theta) \\
\sin (\theta) & \cos (\theta)
\end{array}\right)
$$

Then by choosing a specific reference coordinate system for the 2-component spin space in which the matrix $Q_{a b}^{\mu \nu}$ becomes diagonal, i. .e. $Q_{a b}^{\mu \nu}=q_{a b} \delta_{a b}$, a generic replica field $Q_{a b}^{\mu \nu}$ can be parametrized as,

$$
Q_{a b}^{\mu \nu}=q_{a b} \sum_{\gamma}\left(R^{t}\right)^{\mu \gamma}\left(\theta_{a}\right) R^{\gamma \nu}\left(\theta_{b}\right)=q_{a b}\left(R^{t}\right)^{\mu \nu}\left(\theta_{a}-\theta_{b}\right) .
$$

Using the latter parametrization the free-energy functional Eq. (C3) becomes,

$$
F\left[Q_{a b}^{\mu \nu}\right]=\int d^{d} r\left[\frac{c}{2} \sum_{a<b} q_{a b}^{2}\left(\nabla\left(\theta_{a}-\theta_{b}\right)\right)^{2}+\frac{c}{2} \sum_{a<b}\left(\nabla q_{a b}\right)^{2}+w\left(q_{a b}^{2}\right)\right] .
$$

Note that the latter two terms of the integrand appear also in the usual scalar replica field theories. On the other hand, the 1st term can be naturally interpreted as the spin-wave term just as in the case of the ferromagnetic model (See Eq. (54) ). 
As discussed in appendix B the matrix $q_{a b}$ of the cloned system can be parametrized as,

$$
q_{a b}=(1-q) \delta_{a b}+q
$$

with $q$ being the self-overlap, i. e. the Edwards-Anderson order parameter. Then the free-energy functional can be simplified as,

$$
F\left[Q_{a b}^{\mu \nu}\right]=\int d^{d} r\left[\frac{1}{2} \sum_{a, b} \mu_{a b} \nabla \theta_{a} \nabla \theta_{b}+\frac{c}{2} \sum_{a<b}(\nabla q)^{2}+w\left(q^{2}\right)\right]
$$

Here $\mu_{a b}$ is the rigidity matrix of the spin-waves which is obtained as,

$$
\mu_{a b}=c m q^{2}\left(\delta_{a b}-\frac{1}{m}\right)
$$

Remarkably the rigidity matrix Eq. (C10) has precisely the same structure as that for the shear-modulus of glasses (see sec. II $): \mu=\hat{\mu} \delta_{a b}+\tilde{\mu}$ with $\tilde{\mu}=-(1 / m) \hat{\mu}$ so that the sum rule $\sum_{b=1}^{m} \mu_{a b}=0$ is satisfied. Now the 'intra-state spin-wave rigidity' can be easily read off as,

$$
\hat{\mu}=c m q^{2}
$$

Evidently this is quite similar to the case of ferromagnet discussed in sec. II B 1 in the sense that the spin-wave rigidity is proportional to the square of the order parameter (here the Edwards-Anderson order parameter $q$ ).

We readily know that the Edwards-Anderson order parameter $q$ jumps discontinuously to 0 approaching the dynamical transition temperature $T_{\mathrm{d}}$ from below, preceded by a square-root singularity,

$$
q(T)-q\left(T_{\mathrm{d}}\right) \propto \sqrt{T_{\mathrm{d}}-T}
$$

Using the latter in Eq. (C11), we immediately find that the 'intra-state spin-wave rigidity' should discontinuously vanish approaching $T_{\mathrm{d}}$ from below preceded by a square-root singularity,

$$
\hat{\mu}(T)-\hat{\mu}\left(T_{\mathrm{d}}\right) \propto c m \sqrt{T_{\mathrm{d}}-T}
$$

where $m=1$ at $T_{\mathrm{SG}}<T<T_{\mathrm{d}}$. Apparently these features are the same as the superheated ferromagnet discussed in sec. II B 1.

\section{Appendix D: Proof of the relation Eq. (148)}

Suppose that the original 2-body interaction potential $v(r)$ (See Eq. (4)) is slightly deformed as $v(r) \rightarrow v(r)+\delta w(r)$ where $w(r)$ is an additional potential and $\delta$ is a small parameter. Notice a trivial fact that the rigidity Eq. (8) must be zero irrespective of the deformation of the potential as long as the system remains in the liquid phase. Then the identity,

$$
\langle b\rangle-\beta\left[\left\langle\sigma^{2}\right\rangle-\langle\sigma\rangle^{2}\right]=0
$$

must continue to hold under variation of the parameter $\delta$. Especially the form of the renormalized potential $v_{\text {eff }}(r)$ given in Eq. (94) suggests us to consider the case $w(r)=\nabla^{2} v(r)$.

Let us examine changes of the thermal averages on the l. h. s. of Eq. (D1) under variation of the parameter $\delta$. From the microscopic definitions of the shear-stress $\sigma$ and the Born term $b$ given in Eq. (77) and Eq. (9), we find that they are actually derivatives of the potential $v(r)$ so that they change under variation of $\delta$. Thus the thermal averages can expressed formally as,

$$
\left\langle O_{[v(r)+\delta w(r)]}\right\rangle=Z^{-1} \int_{\mathcal{V}} \prod_{i=1}^{N} \frac{d^{d} r_{i}}{\Lambda^{d}} e^{-\beta \sum_{i<j} v(r)+\delta w(r)} O_{[v(r)+\delta w(r)]} \quad Z=\int_{\mathcal{V}} \prod_{i=1}^{N} \frac{d^{d} r_{i}}{\Lambda^{d}} e^{-\beta \sum_{i<j} v(r)+\delta w(r)}
$$

with $O$ being the Born term $b$, the shear-stress $\sigma$ or $\sigma^{2}$. Expanding Eq. (D2) in power series of $\delta$ and inserting the results into Eq. (D1), we can find an identity at each order of $\delta$. 
Choosing the additional potential in particular as $w(r)=\nabla^{2} v(r)$, we find at $O(\delta)$ the following identity,

$$
\begin{aligned}
0= & \frac{1}{N} \sum_{i<j}\left\langle\nabla^{2} b\left(\mathbf{r}_{i j}\right)\right\rangle-\frac{1}{N} \sum_{i<j} \sum_{k<l} \beta\left[\left\langle b\left(\mathbf{r}_{i j}\right) \nabla^{2} v\left(r_{k l}\right)\right\rangle-\left\langle b\left(\mathbf{r}_{i j}\right)\right\rangle\left\langle\nabla^{2} v\left(r_{k l}\right)\right\rangle\right] \\
& -2 \frac{1}{N} \sum_{i_{1}<j_{1}} \sum_{i_{2}<j_{2}} \beta\left[\left\langle\nabla^{2} \sigma\left(\mathbf{r}_{i_{1} j_{1}}\right) \sigma\left(\mathbf{r}_{i_{2} j_{2}}\right)\right\rangle-\left\langle\nabla^{2} \sigma\left(\mathbf{r}_{i_{1} j_{1}}\right)\right\rangle\left\langle\sigma\left(\mathbf{r}_{i_{2} j_{2}}\right)\right\rangle\right] \\
& +\frac{1}{N} \sum_{i_{1}<j_{1}} \sum_{i_{2}<j_{2}} \sum_{k<l} \beta \beta\left[\left\langle\sigma\left(\mathbf{r}_{i_{1} j_{1}}\right) \sigma\left(\mathbf{r}_{i_{2} j_{2}}\right) \nabla^{2} v\left(r_{k l}\right)\right\rangle-\left\langle\sigma\left(\mathbf{r}_{i_{1} j_{1}}\right) \sigma\left(\mathbf{r}_{i_{2} j_{2}}\right)\right\rangle\left\langle\nabla^{2} v\left(r_{k l}\right)\right\rangle\right]
\end{aligned}
$$

Using the above result for the liquid at temperature $T / m^{*}$, we find the relation Eq. (148),

$$
J_{2}+J_{3}+m^{*}\left(J_{4}+J_{5}\right)=0 .
$$

\section{Appendix E: Representation in terms of particle distribution functions}

In order to compute each terms in Eq. (152), we need to evaluate thermal averages $\langle\ldots\rangle_{*}$ over the thermal fluctuations of several quantities which are multi-point functions of the CM coordinates of the molecules. To this end it is convenient to represent each terms in Eq. (152) and related quantities in terms of particle distribution functions,

$$
\rho^{2} g_{*}^{(2)}\left(\mathbf{r}, \mathbf{r}^{\prime}\right)=\sum_{i \neq j}\left\langle\delta^{d}\left(\mathbf{r}_{i}-\mathbf{r}\right) \delta^{d}\left(\mathbf{r}_{j}-\mathbf{r}^{\prime}\right)\right\rangle_{*} \quad \rho^{3} g_{*}^{(3)}\left(\mathbf{r}, \mathbf{r}^{\prime}, \mathbf{r}^{\prime \prime}\right)=\sum_{i \neq j \neq k}\left\langle\delta^{d}\left(\mathbf{r}_{i}-\mathbf{r}\right) \delta^{d}\left(\mathbf{r}_{j}-\mathbf{r}^{\prime}\right) \delta^{d}\left(\mathbf{r}_{k}-\mathbf{r}^{\prime \prime}\right)\right\rangle_{*},
$$

where $\rho=N / V$ is the number density. Here we introduced a short-hand notations $\sum_{i \neq j}=\sum_{i} \sum_{j}\left(1-\delta_{i j}\right), \sum_{i \neq j \neq k}=$ $\sum_{i} \sum_{j} \sum_{k}\left(1-\delta_{i j} \delta_{j k}\right)$. Since we will consider homogeneous and isotropic systems, we can write,

$$
\begin{aligned}
& \rho^{2} g(r)=\frac{\rho}{N} \sum_{i \neq j}\left\langle\delta^{d}\left(\mathbf{r}_{i j}-\mathbf{r}\right)\right\rangle \\
& \rho^{3} g^{(3)}\left(\mathbf{r}, \mathbf{r}^{\prime}\right)=\frac{\rho}{N} \sum_{i \neq j \neq k}\left\langle\delta^{d}\left(\mathbf{r}_{i j}-\mathbf{r}\right) \delta^{d}\left(\mathbf{r}_{i k}-\mathbf{r}^{\prime}\right)\right\rangle=\sum_{l=0}^{\infty} g_{l}^{(3)}\left(r, r^{\prime}\right) P_{l}(\cos (\theta)) \quad \cos (\theta)=\frac{\mathbf{r} \cdot \mathbf{r}^{\prime}}{r r^{\prime}}
\end{aligned}
$$

In the first equation $g(r)=g^{(2)}(r)$ is the usual radial distribution function. In the last equation $P_{l}(x)(l=0,1,2, \ldots)$ are the Legendre polynomials $\left(P_{0}(x)=1, P_{1}(x)=x, P_{2}(x)=\frac{1}{2}\left(3 x^{2}-1\right), \ldots\right)$ and $\theta$ is the angle between vector $\mathbf{r}$ and $\mathbf{r}^{\prime}$. The coefficients $g_{l}^{(3)}\left(r, r^{\prime}\right)$ are defined as,

$$
g_{l}^{(3)}\left(r, r^{\prime}\right) \equiv \frac{2}{2 l+1} \int_{0}^{\pi} d \theta \sin (\theta) P_{l}(\cos (\theta)) g^{(3)}\left(\mathbf{r}, \mathbf{r}^{\prime}\right) .
$$

The radial distribution function $g(r)$ can be computed using the standard closures of the liquid theory [23] such as the hyper-netted-chain approximation (HNC) [110] and e.t.c. A simple way to evaluate the three-point correlation function $g^{(3)}\left(\mathbf{r}, \mathbf{r}^{\prime}\right)$ is the Kirkwood superposition approximation,

$$
g^{(3)}\left(\mathbf{r}, \mathbf{r}^{\prime}\right)=g(r) g\left(r^{\prime}\right) g\left(\left|\mathbf{r}-\mathbf{r}^{\prime}\right|\right) .
$$

1. Cage size

The cage size $A$ at the 1st order cage expansion Eq. (104) of $d=3$ dimensional system can be expressed as,

$$
A=\frac{3}{\beta \rho \int_{0}^{\infty} d r 4 \pi r^{2} g_{*}(r) \nabla^{2} v(r)}
$$

with the radial distribution function $g_{*}(r)$ at temperature $T^{*}=T / m^{*}(T)$ and,

$$
\nabla^{2} v(r)=v^{(2)}(r)+2 \frac{v^{(1)}}{r} .
$$


2. Affine response

Similarly the Born term can also be expressed just by the radial distribution function $g(r)$ as,

$$
\begin{aligned}
\left\langle b_{\left[\left\{\mathbf{R}_{i}\right\}\right]}\right\rangle_{*} & =\frac{1}{N} \sum_{i<j}\left\langle b\left(\mathbf{r}_{i j}\right)\right\rangle_{*}=\frac{\rho}{2} \int_{0}^{\infty} d r 4 \pi r^{2} g_{*}(r)\left(\left(r^{2} v^{(2)}-r v^{(1)}\right)\left\langle\hat{x}^{2} \hat{z}^{2}\right\rangle_{\text {angle }}+r v^{(1)}\left\langle\hat{z}^{2}\right\rangle_{\text {angle }}\right) \\
& =\frac{\rho}{2} \int_{0}^{\infty} d r 4 \pi r^{2} g_{*}(r)\left(\frac{1}{15} r^{2} v^{(2)}+\frac{4}{15} r v^{(1)}\right)
\end{aligned}
$$

where we used $b(\mathbf{r})=r^{2} v^{(2)} \hat{x}^{2} \hat{z}^{2}+r v^{(1)}\left(1-\hat{x}^{2}\right) \hat{z}^{2}$ given in Eq. (9). Here $\langle\ldots\rangle$ angle denotes an "angular average" over isotropic fluctuations of the orientations of the unit vectors $(\hat{x}, \hat{y}, \hat{z})=(x / r, y / r, z / r)$ with $r=$ $\sqrt{x^{2}+y^{2}+z^{2}}$.

The terms $J_{2}$ and $J_{3}$ are corrections to the Born term due to the renormalization of the potential Eq. (94). The term $J_{2}$ just involves 2-particle distribution function and we find,

$$
J_{2}=-c \frac{1}{N} \sum_{i<j}\left\langle\nabla^{2} b\left(\mathbf{r}_{i j}\right)\right\rangle_{*}=-c \frac{\rho}{2} \int_{0}^{\infty} d r 4 \pi r^{2} g_{*}(r)\left[\frac{1}{15} r^{2} v^{(4)}(r)+\frac{2}{3} r v^{(3)}(r)+\frac{22}{15} v^{(2)}(r)+\frac{8}{15} \frac{v^{(1)}(r)}{r}\right]
$$

On the other hand $J_{3}$ is more complicated since it involves connected 4-particle distribution function. This term can be evaluated by considering a liquid with a modified potential,

$$
v_{\text {eff }}(r ; \delta)=v(r)+\delta \nabla^{2} v(r),
$$

at temperature $T^{*}=T / m$. Let us denote its radial distribution function as $g_{*}(r ; \delta)$. Then

$$
\begin{aligned}
& J_{3}=c \frac{1}{N} \sum_{i<j} \sum_{k<l}\left[\left\langle b\left(\mathbf{r}_{i j}\right) \beta^{*} \nabla^{2} v\left(r_{k l}\right)\right\rangle_{*}-\left\langle b\left(\mathbf{r}_{i j}\right)\right\rangle_{*}\left\langle\beta^{*} \nabla^{2} v\left(r_{k l}\right)\right\rangle_{*}\right] \\
& =\left.c \frac{\rho}{2} \int_{0}^{\infty} d r 4 \pi r^{2} \frac{\partial g_{*}(r ; \delta)}{\partial \delta}\right|_{\delta=0} b\left(\mathbf{r}_{i j}\right)=\left.c \frac{\rho}{2} \int_{0}^{\infty} d r 4 \pi r^{2} \frac{\partial g_{*}(r ; \delta)}{\partial \delta}\right|_{\delta=0}\left(\frac{1}{15} r^{2} v^{(2)}+\frac{4}{15} r v^{(1)}\right) .
\end{aligned}
$$

3. Non-affine response

Next let us examine the $m J_{1}$ term defined in Eq. (146). By noting $\sum_{i} \sum_{j_{1}(\neq i)} \sum_{j_{2}(\neq i)}=\sum_{i \neq j}+\sum_{i \neq j_{1} \neq j_{2}}$, the term $m J_{1}$ can be decomposed into two parts as,

$$
\begin{aligned}
m J_{1} & =c \frac{1}{N} \sum_{i} \sum_{j_{1}(\neq i)} \sum_{j_{2}(\neq i)} \beta^{*}\left\langle\nabla \sigma\left(\mathbf{r}_{i j_{1}}\right) \cdot \nabla \sigma\left(\mathbf{r}_{i j_{2}}\right)\right\rangle_{*}=c \frac{1}{N} \sum_{i \neq j} \beta^{*}\left\langle\left|\nabla \sigma_{i j}\right|^{2}\right\rangle_{*}+c \frac{1}{N} \sum_{i \neq j_{1} \neq j_{2}} \beta^{*}\left\langle\nabla \sigma\left(\mathbf{r}_{i j_{1}}\right) \cdot \nabla \sigma\left(\mathbf{r}_{i j_{2}}\right)\right\rangle_{*} \\
& =\underbrace{c \beta^{*} \rho \int d^{d} r g_{*}(r)|\Xi(\mathbf{r})|^{2}}_{m J_{11}}+\underbrace{c \beta^{*} \rho^{2} \int d^{d} r_{1} d^{d} r_{2} g_{*}^{(3)}\left(\mathbf{r}_{1}, \mathbf{r}_{2}\right) \Xi\left(\mathbf{r}_{1}\right) \cdot \Xi\left(\mathbf{r}_{2}\right)}_{m J_{12}}
\end{aligned}
$$

Using Eq. (77) we find the explicit expression of the vector $\Xi=\left(\Xi_{x}, \Xi_{y}, \Xi_{z}\right)$,

$$
\begin{aligned}
& \boldsymbol{\Xi}_{x}(\mathbf{r})=\frac{\partial \sigma(\mathbf{r})}{\partial x}=\left[r v^{(2)}(r) \hat{x}^{2}+v^{(1)}\left(1-\hat{x}^{2}\right)\right] \hat{z} \\
& \boldsymbol{\Xi}_{y}(\mathbf{r})=\frac{\partial \sigma(\mathbf{r})}{\partial y}=\left[r v^{(2)}(r)-v^{(1)}(r)\right] \hat{x} \hat{y} \hat{z} \\
& \boldsymbol{\Xi}_{z}(\mathbf{r})=\frac{\partial \sigma(\mathbf{r})}{\partial z}=\left[r v^{(2)}(r) \hat{z}^{2}+v^{(1)}\left(1-\hat{z}^{2}\right)\right] \hat{x} .
\end{aligned}
$$

The 1st term in the r.h.s of the last equation of Eq. (E12), i. .e $m J_{11}$ can be easily evaluated using the radial distribution function $g(r)$,

$$
\left.\left.\left.m J_{11}=c \beta^{*} \rho \int d^{d} r g_{*}(r)|\Xi(\mathbf{r})|^{2}=c \beta^{*} \rho \int d r 4 \pi r^{2} g_{*}(r)\left[\frac{1}{15} r v^{(2)}(r)\right)^{2}+\frac{2}{15} r v^{(2)}(r) v^{(1)}(r)\right)+\frac{7}{15} v^{(1)}(r)\right)^{2}\right] .
$$


The 2 nd term in the r.h.s of the last equation of Eq. E12), i. .e $m J_{12}$ involves the 3-particle distribution function $g^{(3)}\left(\mathbf{r}, \mathbf{r}^{\prime}\right)$. As shown in Appendix $\mathrm{E}$ it can be cast into the following form,

$$
\begin{aligned}
& m J_{12}=c \beta^{*} \rho^{2} \int d^{d} r_{1} d^{d} r_{2} r_{1}^{2} r_{2}^{2} g_{*}^{(3)}\left(\mathbf{r}_{1}, \mathbf{r}_{2}\right) \Xi\left(\mathbf{r}_{1}\right) \cdot \Xi\left(\mathbf{r}_{2}\right) \\
& =c \beta^{*} \rho^{2} \int d r_{1} d r_{2} r_{2} r_{1}^{2} r_{2}^{2}\left[\left(g_{1}^{(3}\right)_{*}\left(r_{1}, r_{2}\right) \frac{32 \pi^{2}}{9}\left(\frac{v^{(1)}\left(r_{1}\right)-r_{1} v^{(2)}\left(r_{1}\right)}{5}-v^{(1)}\left(r_{1}\right)\right)\left(\frac{v^{(1)}\left(r_{2}\right)-r_{2} v^{(2)}\left(r_{2}\right)}{5}-v^{(1)}\left(r_{2}\right)\right)\right. \\
& \left.+\left(g_{2}^{(3)}\right)_{*}\left(r_{1}, r_{2}\right) \frac{16 \pi^{2}}{175}\left(r_{1} v^{(2)}\left(r_{1}\right)-v^{(1)}\left(r_{1}\right)\right)\left(r_{2} v^{(2)}\left(r_{2}\right)-v^{(1)}\left(r_{2}\right)\right)\right]
\end{aligned}
$$

where $g_{1}^{(3)}\left(r_{1}, r_{2}\right)$ and $g_{2}^{(3)}\left(r_{1}, r_{2}\right)$ are the coefficients of of the expansion of the three particle correlation function $g^{(3)}\left(\mathbf{r}_{1}, \mathbf{r}_{2}\right)$ by the Legendre polynomials (see Eq. (E3) $)$.

\section{Appendix F: Evaluation of $m J_{12}$}

Here we show derivation of the 2 nd equation of Eq. E15). The term $m J_{12}$ is defined as,

$$
m J_{12} \equiv c \beta^{*} \rho^{2} \int d^{d} r_{1} d^{d} r_{2} g_{*}^{(3)}\left(\mathbf{r}_{1}, \mathbf{r}_{2}\right) \boldsymbol{\Xi}\left(\mathbf{r}_{1}\right) \cdot \boldsymbol{\Xi}\left(\mathbf{r}_{2}\right)
$$

By using the formal expansion of the three particle distribution function by the Legendre polynomials,

$$
g^{(3)}\left(\mathbf{r}_{1}, \mathbf{r}_{2}\right)=\sum_{l=0}^{\infty} g_{l}^{(3)}\left(r_{1}, r_{2}\right) P_{l}(\cos \theta) \quad \cos \theta=\frac{\mathbf{r} \cdot \mathbf{r}^{\prime}}{r r^{\prime}}
$$

and a formal expansion of the field $\boldsymbol{\Xi}(\mathbf{r})$ by spherical harmonics $Y_{n}^{m}(\theta, \phi)$,

$$
\boldsymbol{\Xi}(\mathbf{r})=\sum_{n} \sum_{m} \mathbf{C}_{n}^{m}(\mathbf{r}) Y_{n}^{m}(\theta, \phi)
$$

where $\theta$ and $\phi$ are the angular variables of the 3 -dimensional polar coordinate $\mathbf{r}=r(\sin \theta \cos \phi, \sin \theta \sin \phi, \cos \theta)$, Eq. (F1) can be rewritten as,

$$
m J_{12}=c \beta^{*} \int d r_{1} \int d r_{2} r_{1}^{2} r_{2}^{2} \sum_{l}\left(g_{l}^{(3)}\right)_{*}\left(r_{1}, r_{2}\right) \frac{4 \pi}{2 l+1} \sum_{m=-l}^{l} \mathbf{C}_{l}^{m}(\mathbf{r}) \cdot\left[\mathbf{C}_{l}^{m}\left(r_{2}\right)\right]^{*}
$$

where $[\ldots]^{*}$ means the complex conjugate.

Explicit expressions of the components of the vector field $\Xi()$ is given in Eq. (E13). From the latter we find, the coefficients $\mathbf{C}_{n}^{m}$ of their expansions by the spherical harmonics as,

$$
\begin{aligned}
& \left(\mathbf{C}_{l}^{m}(\mathbf{r})\right)_{x}=\left(\mathbf{C}_{l}^{m}(\mathbf{r})\right)_{z}=\delta_{l, 1} \delta_{m, 0} \sqrt{\frac{4 \pi}{3}}\left\{\frac{1}{5}\left(-r v^{(2)}+v^{(1)}\right)-v^{(1)}\right\}+\delta_{l, 3} \delta_{m, 0} \sqrt{\frac{4 \pi}{7}}\left\{-\frac{1}{5}\left(-r v^{(2)}+v^{(1)}\right)\right\} \\
& +\delta_{l, 3}\left(\delta_{m, 2}-\delta_{m,-2}\right) \sqrt{\frac{4 \pi}{7}}\left\{\frac{\sqrt{5 !}}{60}\left(-r v^{(2)}+v^{(1)}\right)\right\} \\
& \left(\mathbf{C}_{l}^{m}(\mathbf{r})\right)_{y}=\sqrt{-1} \delta_{l, 3}\left(\delta_{m, 2}+\delta_{m,-2}\right) \sqrt{\frac{4 \pi}{7}}\left\{\frac{\sqrt{5 !}}{60}\left(-r v^{(2)}+v^{(1)}\right)\right\} .
\end{aligned}
$$

Using the above result in Eq. (F4) we obtain Eq. (E15) which reads,

$$
\begin{aligned}
& m J_{12}=c \beta^{*} \rho^{2} \int d r_{1} d r_{2} r_{1}^{2} r_{2}^{2}\left[\left(g_{1}^{(3}\right)_{*}\left(r_{1}, r_{2}\right) \frac{32 \pi^{2}}{9}\left(\frac{v^{(1)}\left(r_{1}\right)-r_{1} v^{(2)}\left(r_{1}\right)}{5}-v^{(1)}\left(r_{1}\right)\right)\left(\frac{v^{(1)}\left(r_{2}\right)-r_{2} v^{(2)}\left(r_{2}\right)}{5}-v^{(1)}\left(r_{2}\right)\right)\right. \\
& \left.+\left(g_{2}^{(3)}\right)_{*}\left(r_{1}, r_{2}\right) \frac{16 \pi^{2}}{175}\left(r_{1} v^{(2)}\left(r_{1}\right)-v^{(1)}\left(r_{1}\right)\right)\left(r_{2} v^{(2)}\left(r_{2}\right)-v^{(1)}\left(r_{2}\right)\right)\right] .
\end{aligned}
$$




\section{Appendix G: Formulations for the binary mixture}

Following Coluzzi et. al. in [19] we denote the radial distribution function between particles of types $\epsilon=+,-$ and $\epsilon^{\prime}=+,-$ as $g^{\epsilon \epsilon^{\prime}}(r)$. At the order of 1st order cage expansion, the cage sizes of the two types of the particles are found to be,

$$
A_{\epsilon}=\frac{3}{\beta \rho \int_{0}^{\infty} d r 4 \pi r^{2} \sum_{\epsilon^{\prime}} x_{\epsilon^{\prime}} g_{*}^{\epsilon \epsilon^{\prime}}(r) \nabla^{2} v^{\epsilon \epsilon^{\prime}}(r)} .
$$

which is a generalization of Eq. (E6).

The Born term Eq. (E8) becomes in the binary case,

$$
\left\langle b_{\left[\left\{\mathbf{R}_{i}\right\}\right]}\right\rangle_{*}=\frac{\rho}{2} \sum_{\epsilon \epsilon^{\prime}} \int_{0}^{\infty} d r 4 \pi r^{2} x_{\epsilon} x_{\epsilon^{\prime}} g_{*}^{\epsilon \epsilon^{\prime}}(r)\left(\frac{1}{15} r^{2}\left(v^{\epsilon \epsilon^{\prime}}\right)^{(2)}+\frac{4}{15} r\left(v^{\epsilon, \epsilon^{\prime}}\right)^{(1)}\right) .
$$

Similarly the $J_{2}$ term Eq. (E9) becomes,

$$
J_{2}=-\sum_{\epsilon \epsilon^{\prime}} c_{\epsilon} \frac{\rho}{2} \int_{0}^{\infty} d r 4 \pi r^{2} x_{\epsilon} x_{\epsilon^{\prime}} g_{*}^{\epsilon \epsilon^{\prime}}(r)\left[\frac{1}{15} r^{2}\left(v^{\epsilon \epsilon^{\prime}}\right)^{(4)}(r)+\frac{2}{3} r\left(v^{\epsilon \epsilon^{\prime}}\right)^{(3)}(r)+\frac{22}{15}\left(v^{\epsilon \epsilon^{\prime}}\right)^{(2)}(r)+\frac{8}{15} \frac{\left(v^{\epsilon \epsilon^{\prime}}\right)^{(1)}(r)}{r}\right]
$$

where the parameters $c_{\epsilon}$ are related to the cages sizes $A_{\epsilon}$ as,

$$
c_{\epsilon}=2 \frac{A_{\epsilon}}{m},
$$

which is a generalization of Eq. (147). Similarly the $J_{3}$ term Eq. (E11) becomes,

$$
J_{3}=\left.\sum_{\epsilon} c_{\epsilon} \frac{\rho}{2} \int_{0}^{\infty} d r 4 \pi r^{2} \sum_{\epsilon^{\prime} \epsilon^{\prime \prime}} x_{\epsilon^{\prime}} x_{\epsilon^{\prime \prime}} \frac{\partial g_{*}^{\epsilon^{\prime} \epsilon^{\prime \prime}}\left(r ; \delta_{+}, \delta_{-}\right)}{\partial \delta_{\epsilon}}\right|_{\delta=0}\left(\frac{1}{15} r^{2}\left(v^{\epsilon^{\prime} \epsilon^{\prime \prime}}\right)^{(2)}+\frac{4}{15} r\left(v^{\epsilon^{\prime} \epsilon^{\prime \prime}}\right)^{(1)}\right),
$$

where $g^{\epsilon^{\prime} \epsilon^{\prime \prime}}\left(r ; \delta_{+}, \delta_{-}\right)$is the radial distribution function of a system with modified potential,

$$
v_{\mathrm{eff}}^{\epsilon_{1} \epsilon_{2}}\left(r ; \delta_{+}, \delta_{-}\right)=v^{\epsilon_{1} \epsilon_{2}}(r)+\sum_{\epsilon_{3}=+,-} \delta_{\epsilon_{3}} \nabla^{2} v^{\epsilon_{3} \epsilon_{2}}(r) .
$$

The non-affine correction term $J_{1}$ Eq. (155) becomes,

$$
m J_{1}=\sum_{\epsilon= \pm} c_{\epsilon} \beta^{*} \Xi_{\epsilon}^{2}
$$

where

$$
\Xi_{\epsilon}^{2} \equiv \frac{1}{N_{\epsilon}} \sum_{i \in \epsilon}\left\langle\left|\sum_{j(\neq i)} \nabla \sigma\left(\mathbf{r}_{i j}\right)\right|^{2}\right\rangle_{*}
$$

with $N_{\epsilon}=N x_{\epsilon}$. Using the particle distribution functions we find $J_{11}$ Eq. (E14) and $J_{12}$ Eq. (E15) become,

$$
\begin{aligned}
& \left.\left.\left.m J_{11}=\sum_{\epsilon} c_{\epsilon} \beta^{*} \rho \int d r 4 \pi r^{2} \sum_{\epsilon^{\prime}} x_{\epsilon} x_{\epsilon^{\prime}} g_{*}^{\epsilon \epsilon}(r)\left[\frac{1}{15} r\left(v^{\epsilon \epsilon^{\prime}}\right)^{(2)}(r)\right)^{2}+\frac{2}{15} r\left(v^{\epsilon \epsilon^{\prime}}\right)^{(2)}(r) v^{(1)}(r)\right)+\frac{7}{15}\left(v^{\epsilon \epsilon^{\prime}}\right)^{(1)}(r)\right)^{2}\right] . \\
& m J_{12}=\sum_{\epsilon} c_{\epsilon} \beta^{*} \rho^{2} \int d r_{1} d r_{2} r_{1}^{2} r_{2}^{2} \sum_{\epsilon^{\prime} \epsilon^{\prime \prime}}[ \\
& \left(\left(g^{\epsilon \epsilon^{\prime} \epsilon^{\prime \prime}}\right)_{1}^{(3)}\right)_{*}\left(r_{1}, r_{2}\right) \frac{32 \pi^{2}}{9}\left(\frac{\left(v^{\epsilon \epsilon^{\prime}}\right)^{(1)}\left(r_{1}\right)-r_{1}\left(v^{\epsilon \epsilon^{\prime}}\right)^{(2)}\left(r_{1}\right)}{5}-\left(v^{\epsilon \epsilon^{\prime}}\right)^{(1)}\left(r_{1}\right)\right)\left(\frac{\left(v^{\epsilon \epsilon^{\prime \prime}}\right)^{(1)}\left(r_{2}\right)-r_{2}\left(v^{\epsilon \epsilon^{\prime \prime}}\right)^{(2)}\left(r_{2}\right)}{5}-\left(v^{\epsilon \epsilon^{\prime \prime}}\right)^{(1)}\left(r_{2}\right)\right) \\
& \left.+\left(\left(g^{\epsilon \epsilon^{\prime} \epsilon^{\prime \prime}}\right)_{2}^{(3)}\right)_{*}\left(r_{1}, r_{2}\right) \frac{16 \pi^{2}}{175}\left(r_{1}\left(v^{\epsilon \epsilon^{\prime}}\right)^{(2)}\left(r_{1}\right)-\left(v^{\epsilon \epsilon^{\prime}}\right)^{(1)}\left(r_{1}\right)\right)\left(r_{2}\left(v^{\epsilon \epsilon^{\prime \prime}}\right)^{(2)}\left(r_{2}\right)-\left(v^{\epsilon \epsilon^{\prime \prime}}\right)^{(1)}\left(r_{2}\right)\right)\right]
\end{aligned}
$$


where $\left(g^{\epsilon \epsilon^{\prime} \epsilon^{\prime \prime}}\right)_{l}^{(3)}\left(r_{1}, r_{2}\right)$ with $l=1,2, \ldots$ are the coefficients of the expansion of the three-particle distribution function by the Legendre polynomials (See Eq. (E3)). Using the Kirkwood superposition approximation Eq. (E5) we evaluate them as,

$$
\left(g^{\epsilon \epsilon^{\prime} \epsilon^{\prime \prime}}\right)_{l}^{(3)}\left(r, r^{\prime}\right)=\frac{2}{2 l+1} \int_{0}^{\pi} d \theta \sin (\theta) P_{l}(\cos (\theta)) g^{\epsilon \epsilon^{\prime}}(r) g^{\epsilon^{\prime} \epsilon^{\prime \prime}}\left(r^{\prime}\right) g^{\epsilon^{\prime \prime} \epsilon}\left(\left|\mathbf{r}-\mathbf{r}^{\prime}\right|\right) \quad \cos (\theta)=\frac{\mathbf{r} \cdot \mathbf{r}^{\prime}}{r r^{\prime}}
$$

[1] P. W. Anderson, Science 117, 393 (1972).

[2] L. D. Landau and E. M. Lifshitz, Theory of Elasticity, 3rd ed. (Pergamon, New York, 1986).

[3] A. Angell, K. L. Ngai, G. B. McKenna, P. F. McMillan, S. W. Martin, J. of App. Phys. 88, 3113 (2000).

[4] L. Berthier and G. Biroli, Rev. of Mod. Phys. 83, 587 (2011).

[5] T. R. Kirkpatrick and D. Thirumalai, Phys. Rev. Lett. 58, 2091 (1987).

[6] T. R. Kirkpatrick and P. G. Wolynes, Phys. Rev. B 36, 8552 (1987).

[7] T. R. Kirkpatrick, D. Thirumalai, and P. G. Wolynes, Phys. Rev. A 40, 1045 (1989).

[8] A. Cavagna, Phys. Rep. 476, 51 (2009).

[9] G. Biroli and J. -P. Bouchaud, The Random First-Order Transition Theory of Glasses: a critical assessment in Structural Glasses and Supercooled Liquids: Theory, Experiment, and Applications Eds: P. G. Wolynes, V. Lubchenko, Wiley (2012), (preprint arXiv:0912.2542).

[10] A.W. Kauzmann, Chem.Rev 43, 219 (1948).

[11] W. Götze and L. Sjögren, Rep. Prog. Phys. 55, 241 (1992).

[12] W. Götze, in: J. P. Hanssen, D. Levesque, J. Zinn-Justin (Eds.), Liquids, Freezing and Glass transition, North Holland, Amsterdam, 1991 p.287.

[13] J. H. Gibbs and E. A. DiMarzio, J. Chem. Phys. 28, 373 (1958).

[14] G. Adam and J.H. Gibbs, J. Chem. Phys. 43, 139 (1965).

[15] M.P. Eastwood and P.G. Wolynes, Europhys. Lett. 60, 587 (2002).

[16] J.-P. Bouchaud, G. Biroli, J. of Chem. Phys. 121, 7347 (2004).

[17] G. Biroli, J. -P. Bouchaud, A. Cavagna, T. S. Grigera, and P. Verrocchio, Nature Physics 4, 771 (2008).

[18] M. Mézard and G. Parisi, Phys. Rev. Lett. 82, 747 (1999) and J. of Chem. Phys, 1111076 (1999).

[19] B. Coluzzi, M. Mézard, G. Parisi and P. Verrochio, J. of Chem. Phys, 1119039 (1999).

[20] G. Parisi and F. Zamponi, Rev. Mod. Phys. 82, 789 (2010).

[21] L. Berthier, H. Jacquin and Z. Zamponi, Phys. Rev. Lett. 106, 135702 (2011) and Phys. Rev. E 84, 051103 (2011).

[22] A. Ikeda and K. Miyazaki, Phys. Rev. Lett. 104, 255704 (2010).

[23] J.-P. Hansen and I. R. Mcdonald, "Theory of simple liquids", Academic Press (2006).

[24] R. Monasson, Phys. Rev. Lett. 75, 2847 (1995).

[25] S. Franz and G. Parisi, J. de Physique 5, 1401 (1995).

[26] D. R. Squire, A. C. Holt, and W. G. Hoover, Physica, 42, 388 (1969).

[27] M. Goldstein, J. Chem. Phys. 51, 3728 (1969).

[28] A. S. Argon, Acta Metall. 27, 47 (1979).

[29] J. C. Dyre, Rev. Mod. Phys. 78, 953 (2006).

[30] J. D. Eshelby, Proc. R. Soc. London, Ser. A 241, 376 (1957).

[31] V. V. Bulatov and A. S. Argon, J. of Non-Crystalline Solids 174, 876 (1994).

[32] C. Maloney and A. Lemaître, Phys. Rev. Lett. 93, 016001 (2004).

[33] C. Maloney and A. Lemaître, Phys. Rev. E 74, 016118 (2006).

[34] F. Corberi, L. F. Cugliandolo and H. Yoshino, Growing length scales in aging systems, in [118], (preprint arXiv:1010.0149).

[35] M. Born and K. Huang, Dynamical Theory of Crystal Lattices, Clarendon Press, Oxford, (1954).

[36] J. L. Barrat, J. -N. Roux, J.-P. Hansen and M. L. Klein, Europhys. Lett. 7 (1988) 707.

[37] M. Born, J. Chem. Phys 7, 591 (1939).

[38] J. L. Tallon, Nature 342, 658 (1989).

[39] J. Wang, J. Li, S. Yip, D. Wolf and S. Phillpot, Physica A 240, 396 (1997).

[40] V. Sorkin, E. Polturak and J. Adler, Phys. Rev B 68, 174102 (2003).

[41] Z. Jin, P. Gumbsch, K. Lu, E. Ma, Phys. Rev. Lett. 87, 055703 (2001).

[42] F. Krzakala and L. Zdeborova, J. Chem. Phys. 134, 034512 (2011) and J. Chem. Phys. 134, 034513 (2011).

[43] T. S. Grigera, A. Cavagna, I. Giardina, and G. Parisi, Phys. Rev. Lett. 88, 055502 (2002).

[44] P. M. Goldbart and N. Goldenfeld, Phys. Rev. A 391402 (1989).

[45] P. M. Goldbart, H. E. Castillo, A. Zippelius, Advances in Physics 45, 58 (1996).

[46] H. E. Castillo and P. M. Goldbart, Phys. Rev. E 62, 8159 (2000).

[47] M. Wyart, Ann. Phys. (Paris) 30, 1 (2005).

[48] M. V. Hecke, J. Phys.: Condens. Matter, 22033101 (2010).

[49] H. Yoshino and M. Mézard, Phys. Rev. Lett. 105, 015504 (2010).

[50] O. Penrose, Markov Processes and Related Fields 8, 351 (2002). 
[51] F. Sauset, G. Biroli and J. Kurchan, J. of Stat. Phys. 140, 718 (2010).

[52] P. M. Chaikin and T. C. Lubensky, Principles of Condensed Matter Physics, Cambridge Univ. Press, (2000).

[53] A. Zaccone and E. Scossa-Romano, Phys. Rev. B 83, 184205 (2011).

[54] S. R. Williams and D. J. Evans, J. Chem. Phys. 131, 024115 (2009).

[55] S. R. Williams and D. J. Evans, J. Chem. Phys. 132, 184105 (2010).

[56] S. R. Williams, J. Chem. Phys. 135, 131102 (2011).

[57] C. Maggi, B. Jakobsen, T. Christensen, N. B. Olsen, and J. C. Dyre, J. of Phys.Chem. B 112, 16320 (2008).

[58] T. G. Mason, Martin-D. Lacasse, Gary S. Grest, Dov Levine, J. Bibette, D. A. Weitz, Phys. Rev. E 56, 3150 (1997).

[59] X. Shi, A. Mandanici and G. B. McKenna, J. Chem. Phys. 123, 174507 (2005).

[60] G. B. McKenna, T. Narita and F. Lequeux, J. Rheolo. 53, 489 (2009).

[61] R. Kubo, M. Toda and N. Hashitume; Statistical Physics II. Nonequilibrium Statistical Mechanics, Springer-Verlag, 1992.

[62] A. Tanguy, J. P. Wittmer, F. Leonforte and J. -L. Barrat, Phys. Rev. B 66174205 (2002).

[63] C. Maloney and A. Lemaître, Phys. Rev. Lett. 93, 195501 (2004).

[64] F. H. Stillinger and T. A. Weber, Phys. Rev. A 25, 978 (1982).

[65] F. H. Stillinger, Science 2675206 (1995).

[66] B. Doliwa and A. Heuer, Phys. Rev. E 67, 031506 (2003).

67] A. Heuer, Journal of Physics. Condensed Matter : 20, 373101 (2008).

[68] A. M. Puertas, E. Zaccarelli and F. Sciortino, J. Phys. Condens. Matter 17, L271 (2005).

[69] A. Furukawa and H. Tanaka, Phys. Rev. Lett. 103, 135703 (2009).

[70] S. Okamura, H. Yoshino and S. Yukawa, in progress.

[71] L. C. E. Struik, Physical Aging in Amorphous Polymers and Other Materials (Elsevier, Amsterdam, 1978).

[72] L. F. Cugliandolo and J. Kurchan, Phys. Rev. Lett. 71, 173 (1993); L. F. Cugliandolo and J. Kurchan, J. Phys. A 27, 5749 (1994).

[73] G. Biroli and R. Monnason, Euro. Phys. Lett. 50, 155 (2000).

[74] A Tanguy, F. Leonforte, and J.-L. Barrat, Eur. Phys. J. E 20, 355 (2006).

[75] A. Lemaitre and C. Maloney, J. Stat. Phys. 123, 415 (2006).

[76] S. Karmakar, A. Lemaître, E. Lerner and I. Procaccia, Phys. Rev. Lett. 104, 215502 (2010).

[77] H. Hentschel, S. Karmakar, E. Lerner, and I. Procaccia, Phys. Rev. E 83, 061101 (2011).

[78] J. F. Lutsko, J. Appl. Phys. 65, 2991 (1989).

[79] J. M. Kosterlitz and D. J. Thouless, J. Phys. C: Solid State Phys. 61181 (1973); J. Kosterlitz J. Phys. C: Solid State Phys. 71046 (1974).

[80] F. Leonforte, R. Boissière, A. Tanguy, J. P. Wittmer, and J.-L. Barrat, Phys. Rev. B 72224206 (2005).

[81] J. Kurchan and L. Laloux, Journal of Physics A: Mathematical and General 29, 1929 (1996).

[82] K. Binder, Rep. Prog. Phys. 50 (1987) 783.

[83] S. Franz and G. Parisi, Phys. Rev. Lett. 79, 2486 (1997),

[84] G. Nägele, J. Bergenholtz, J. Chem. Phys. 108, 9893 (1998).

[85] R. W. Hall and P. G. Wolynes, The Journal of Chemical Physics 86, 2943 (1987).

[86] Y. Singh, J. P. Stoessel, and P. G. Wolynes, Phys. Rev. Lett. 54, 1059 (1985).

[87] T. R. Kirkpatrick and P. G. Wolynes, Phys. Rev. A 35, 3072 (1987).

[88] T. R. Kirkpatrick and D. Thirumalai, J. Phys. Math. Gen. 22, L149 (1989).

[89] S. F. Edwards and P. W. Anderson, Journal of Physics F: Metal Physics 5, 965 (1975).

[90] Castellani and A. Cavagna, Journal of Statistical Mechanics:Theory and Experiment 2005 05012, (2005).

[91] G. Parisi, Phys. Rev. Lett. 50, 1946 (1983).

[92] B. Derrida, Phys. Rev. B 24, 2613 (1981).

[93] H. Yoshino and T. Rizzo, Phys. Rev. B 77, 104429 (2008).

[94] T. Rizzo and H. Yoshino, Phys. Rev. B 73, 064416 (2006).

[95] S. Kirkpatrick and A. P. Young, J. Apple. Phys. 521712 (1981).

[96] A. P. Young, A. J. Bray and M. A. Moore, J. Phys. C 17, L149 (1984).

[97] F. Krzakala and O. C. Martin, Eur. Phys. J. B 28, 199 (2002).

[98] P. Le Doussal, M. Müller, and K. J. Wiese, Euro. Phys. Lett. 91, 57004 (2010).

[99] L. Balents, J. -P. Bouchaud and M. Mézard, J. Phys. I France 6, 1007 (1996).

[100] J. -P. Bouchaud and M. Mézard. J. Phys. A 30, 7997 (1997).

[101] P. Le Doussal and K. J. Wiese, Euro. Phys. Lett., 7766001 (2007).

[102] J. -P. Bouchaud, M. Mézard and G. Parisi, Phys. Rev. E 52, 3656 (1995).

[103] G. Szamel and E. Flenner, Phys. Rev. Lett. 107, 105505 (2011).

[104] G. Kotliar, H. Sompolinsky and A. Zippelius, Phys. Rev. B. 35, 311 (1987).

[105] C. S. O'Hern, L. E. Silbert, A. J. Liu and S. R. Nagel, Phys. Rev. E 68, 011306 (2003).

[106] B. Bernu, J. P. Hansen, Y. Hiwatari, and G. Pastore, Phys. Rev. A 36, 4891 (1987).

[107] J. N. Roux, J. L. Barrat, and Hansen, J. of Phys.: Condensed Matter 1, 7171 (1989).

[108] J. -L. Barrat, J. -N. Roux and J. -P. Hansen, Chem. Phys. 149, 197 (1990).

[109] L. Berthier, private communication.

[110] T. Morita and K. Hiroike, Progr. Theor. Phys. 25, 537 (1961).

[111] H. Yoshino and A. Lemaître, in progress.

[112] S. Abraham and P. Harrowell, arXiv:1201.1343v1. 
[113] J. -L. Barrat and A. Latz, J. Phys. Condens. Matter 2, 4289 (1990).

[114] A. Tanguy, B. Mantisi and M. Tsamados, Euro. Phys. Lett. 90, 16004-01 (2010).

[115] R. Yamamoto and A. Onuki, J. Phys. Soc. Jpn. 662545 (1997).

[116] A. Widmer-Cooper, P. Harrowell. Phys. Rev. Lett, 96185701 (2006).

[117] A. Widmer-Cooper, P. Harrowell. Phys. Rev. E, 80061501 (2009).

[118] Dynamical heterogeneities in glasses, colloids, and granular media, Eds.: L. Berthier, G. Biroli, J. -P. Bouchaud, L. Cipelletti and W. van Saarloos, 2011 (Oxford Univ. Press).

[119] P. Habdas, D. Schaar, A. C. Levitt, and E. R. Weeks, Europhy. Lett. 67, 477 (2004).

[120] G. Parisi, Phys. Rev. Lett. 79, 3660 (1997).

[121] J.-L. Barrat and W. Kob, Europhys. Lett. 46, 637 (1999).

[122] L. Berthier and J.-L. Barrat, J. Chem. Phys. 116, 6228 (2002).

[123] M. Mézard, Physica A 265, 352 (1999).

[124] A. Crisanti and H. -J. Sommers, Z. Phys. B 87, 341 (1992). 